\title{
Emergence of visual content in the human brain : investigations of amblyopia, blindsight and high-level motion perception with fMRI
}

Citation for published version (APA):

Muckli, L. F. (2002). Emergence of visual content in the human brain : investigations of amblyopia, blindsight and high-level motion perception with fMRI. [Doctoral Thesis, Maastricht University]. Shaker Verlag. https://doi.org/10.26481/dis.20020925lm

Document status and date:

Published: 01/01/2002

DOI:

10.26481/dis.20020925Im

Document Version:

Publisher's PDF, also known as Version of record

\section{Please check the document version of this publication:}

- A submitted manuscript is the version of the article upon submission and before peer-review. There can be important differences between the submitted version and the official published version of record.

People interested in the research are advised to contact the author for the final version of the publication, or visit the DOI to the publisher's website.

- The final author version and the galley proof are versions of the publication after peer review.

- The final published version features the final layout of the paper including the volume, issue and page numbers.

Link to publication

\footnotetext{
General rights rights.

- You may freely distribute the URL identifying the publication in the public portal. please follow below link for the End User Agreement:

www.umlib.nl/taverne-license

Take down policy

If you believe that this document breaches copyright please contact us at:

repository@maastrichtuniversity.nl

providing details and we will investigate your claim.
}

Copyright and moral rights for the publications made accessible in the public portal are retained by the authors and/or other copyright owners and it is a condition of accessing publications that users recognise and abide by the legal requirements associated with these

- Users may download and print one copy of any publication from the public portal for the purpose of private study or research.

- You may not further distribute the material or use it for any profit-making activity or commercial gain

If the publication is distributed under the terms of Article $25 \mathrm{fa}$ of the Dutch Copyright Act, indicated by the "Taverne" license above, 
Emergence of Visual Content in the Human Brain Investigations of Amblyopia, Blindsight and High-Level Motion Perception with fMRI

\section{DISSERTATION}

\section{PROEFSCHRIFT}

ter verkrijging van de graad van doctor aan de Universiteit Maastricht, op gezag van de Rector Magnificus, Prof. dr. A.C. Nieuwenhuijzen Kruseman volgens het besluit van het College van Decanen, in het openbaar te verdedigen op woensdag 25 september 2002 om 14.00 uur door Lars Folker Muckli 


\section{Promotiecommissie:}

\section{Promotores:}

Prof. Dr. R. W. Goebel

Prot. Dr. W. Singer

(Max Planck Institute for Brain Research, Frankfurt am Main, Germany)

\section{Beoordelingscommissie:}

Prof. Dr. H. L. G. J. Merckelbach (Voorzitter)

Dr. E. Formisano

Prof. Dr. P. Hagoort (Katholieke Universiteit Nijmegen)

Dr. B. M. Schmitt

Prof. Dr. P. Stoerig (Heinrich-Heine-University, Düsseldort, Germany) 


\section{Berichte aus der Psychologie}

\section{Lars Muckli}

Emergence of Visual Content in the Human Brain Investigations of Amblyopia, Blindsight and High-Level Motion Perception with fMRI 


\section{Die Deutsche Bibliothek - CIP-Einheitsaufnahme}

Muckli, Lars:

Emergence of Visual Content in the Human Brain-Investigations of Amblyopia, Blindsight and High-Level Motion Perception with fMRI/Lars Muckli.

Aachen: Shaker, 2002

(Berichteaus der Psychologie)

Zugl.: University Maastricht, Diss., 2002

ISBN3-8322-0290-0

Copyright Shaker Verlag 2002

All rights reserved. No part of this publication may be reproduced, stored in a retrieval system, or transmitted, in any form or by any means, electronic, mechanical, photocopying, recording or otherwise, without the prior permission of the publishers.

Printed in Germany.

ISBN 3-8322-0290-0

ISSN 0945-0890

Shaker Verlag GmbH • P.O. BOX 1290 - D-52013 Aachen

Phone: 0049/2407/9596-0 - Telefax: 0049/2407/9596-9

Internet: www.shaker.de·eMail: info@shaker.de 
For Ilias 



\section{Table of contents}

$1 \quad$ Introduction

Functional imaging of vision

2

Amblyopia

Cerebral correlates of impaired grating perception in human amblyopia

Blindsight

Sustained extrastriate cortical activation without visual awareness revealed by fMRI studies of hemianopic patients

Higher-level-motion I

Integration of multiple motion vectors over space:

An fMRI study of transparent motion perception

Higher-level-motion II

The constructive nature of vision:

Direct evidence from fMRI studies of apparent motion and motion imagery

Higher-level-motion III

Apparent motion:

Event related fMRI of perceptual switches and states

Summary

Samenvatting

Acknowledgments 


\section{Introduction}

\section{Functional imaging of vision}

This chapter corresponds to:

Lars Muckli ${ }^{1}$ and Rainer Goebel ${ }^{2}$ (in press) Functional imaging of vision. In Francesco DiSale (Eds).NN

${ }^{1}$ Max Planck Institute for Brain Research, Neurophysiology, Frankfurt am Main, Germany ${ }^{2}$ Department of Psychology, Neurocognition, Universiteit Maastricht, Maastricht. The Netherlands. 


\section{Functional imaging of vision}

This chapter is a short neurophysiological introduction to the visual system of humans. Results of non-invasive human brain imaging are taken to illustrate the cortical and subcortical visual processing stages. Selected examples show how some visual features emerge at the respective processing stage. As human brain imaging techniques are restricted in their spatial and temporal resolution it is essential to establish close comparisons to the visual system of animals like monkeys and cats for which other neurophysiological methods can be used. It will be shown that the cortical processing stages in brains of monkeys and humans match nicely for the first few cortical regions, and that variabilities between homologous brain regions increase for higher visual areas. Functionally specialized cortico-cortical pathways were first described in the 80's (Ungerleider and Mishkin, 1982; Mishkin et al., 1983) on grounds of anatomical and electrophysiological animal experiments. Today, functionally specialized pathways can be mapped within the same individuals and compared to individuals of other species using the same brain imaging techniques. 


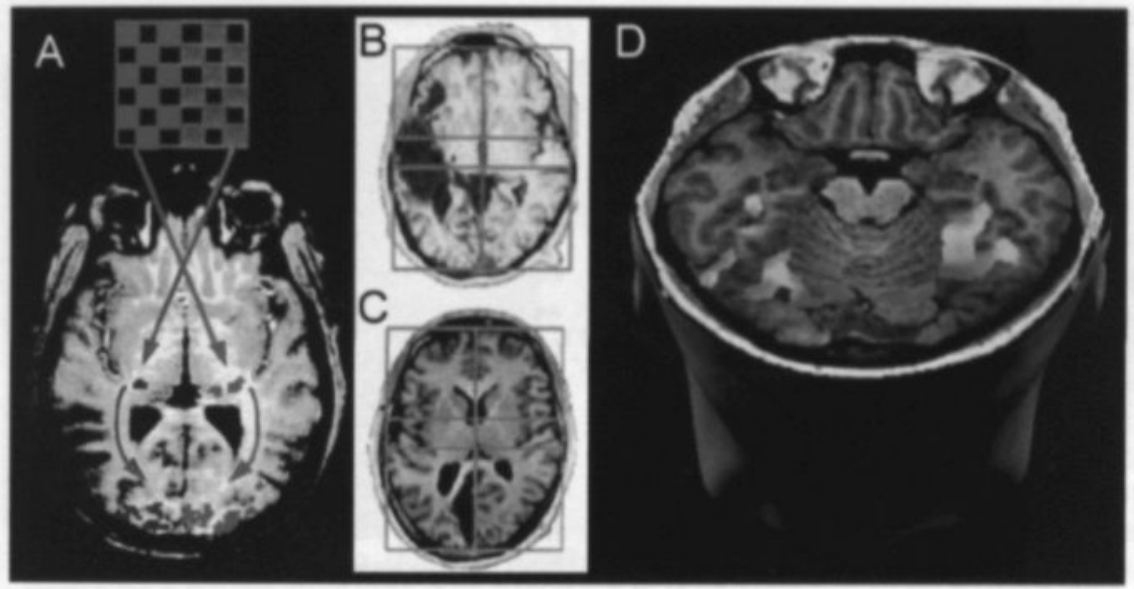

Figure 1. Subcortical and cortical activation of left and right visual field. (A, adapted from Chen et al 1999) Functional images of subcortical activation in the LGN (middle) and in the occipital visual cortex (lower) at high magnetic field strength (4 Tesla) by Chen et al. (2001). Visual stimuli presented in the left visual field is projected to the right hemisphere (red). This rearrangement is organized at the optic chiasm where the nasal ganglion cells cross hemispheres (in this case comming from the left eye). (B, C) Anatomical MRI images of two blindsight patients with lesions affecting (B) the left optic radiation or (C) the primary visual cortex V1 (taken from Goebel et al. 2001). (D) Visualization of brain activity in extrastriate visual areas along the occipito-ventral pathway of a normal subject perceiving visual objects presented in left (green) or right (red) visual field. (control subject in Goebel et al., 2001).

\section{The visual processing stream}

\section{Visual cortex}

The occipital cortex accommodates several visual areas. Each visual area has an entire representation of the visual field. If functional magnetic resonance imaging (IMRI) is used to separate visual areas, a mapping procedure of the visual field representation needs to be utilized (see below). The first visual area (V1) lies in the depth of the calcarine fissure, a prominent anatomical structure in the occipital lobe. The exact location of V1 within the calcarine sulcus can be seen post-mortem in histological slices and in living beings using retinotopic mapping with fMRI. Cytoarchitectonically, V1 (Brodmans area 17, Brodmann, 1905) is characterized by a thick cortical layer IV (the layer which primarily receives afferent input), the stria Genari (Stripe of Genari, Gennari, 1782). Because this stripe is such a distinct structure, V1 is often called the striate cortex whereas the other visual areas form the extrastriate cortex.

\section{Subcortical structure}

Before visual information reaches the cortex light has been transduced to neuronal codes in the photoreceptors of the retina, ganglion cells of the optic nerve project to the thalamus (lateral geniculate nucleus, LGN), from where the signal is passed on to the cortex, mainly to V1. In humans, each eye covers most of the visual field so that $75 \%$ of the visual field can bee seen binocularly. Projec- 
tions from each eye to the LGN are rearranged at the optic chiasm so that all visual information from one side of the visual field is projected to the contralateral hemisphere. This property of the LGN is demonstrated in a fMRI experiment shown in Fig. 1 (left). If one side of the visual field is stimulated the contralateral LGN is activated. It is difficult to acquire functional MR images of the LGN because of its small size and its deep location in the brain. High-field-fMRT (at 4 Tesla field strength) was used to demonstrate retinotopic structure in the LGN (Chen et al., 1999). The optics of the eyes cause the light to be projected up-side-down onto the retina. In the LGN, upper regions respond likewise to the lower visual field and vica versa. Visual information is projected further to the cortex preserving the up-side-down and left-right inverted retinotopy.

Disruption of the projection somewhere between the retina and the cortex leads to circumscribed symptoms: monocularity if anterior to the optic chiasm, blindness in part of one hemifield if a disruption occurs in the optic tract (between optic chiasm and LGN). and cortical blindness for disruption between LGN and V1. Two examples of cortical blindness from our own studies are shown in the middle of Fig. 1 (Goebel et al., 2001). Disruption in the left optic radiation in patient FS (top) and the lesion in the left primary visual cortex (V1) in patient GY (bottom) both lead to blindness in the right visual field (hemianopia). Intriguingly, these patients show residual visual function in the blind hemifield. Both subjects are able to guess the location of objects, or discriminate between objects. without having visual awareness of them when they are presented in their blind hemi. fields. This residual visual function is called blindsight (Weiskrantz et al., 1974). It demonstrates that alternative projection routes from LGN to cortex exist besides the main route to V1. Functional activity can be found in spe- cialized extrastriate visual areas of blindsight subjects if stimulated in their blind field regardless of their own conscious experience.

\section{Retinotopic visual areas}

Before looking more precisely at visual brain activation in the cortex of blindsight subjects, an introduction to the retinotopic visual areas might help to give an orientation to the visual cortex. As mentioned earlier, retinotopic mapping with AMRI is the only method that can identify the extent of V1, and extrastriate visual areas in living humans. It utilizes the fact that every visual area has a full representation of the visual field. If a salient stimulus like the pie-shaped chequerboard in Fig. 2 (a) rotates slowly through the visual field, activity in retinotopic regions migrates continuously through visual areas, preserving the topography of neighborhood relations but magnifying the central visual field known as the cortical magnification factor (Sereno 1995; Popovic 2001). Interestingly the representations from adjacent areas are mirror inverted in the visual cortex. If the stimulation starts at the lower vertical meridian and rotates clockwise to the upper vertical meridian (Fig. 2 a) activity migrates counterclockwise (from a posterior viewpoint) in right hemispheric V1, V3d and VP (green in Fig 2 b) and clockwise in V2d, V2v, V3a and V4 (blue in Fig 2 b; Sereno 1995). This change in activity gradients can be used to separate visual areas graphically as it has been illustrated in Fig. $2 b$.

When the cortical organization is mapped on the flattened cortical sheet of individual hemispheres it can be compared to flattened hemispheres of monkeys that are prepared equally. The cortex is flexible and allows mechanical distortions around $16 \%$ needed in histological and virtual flattening procedures. Size and location of $\mathbf{V} 1$ and $\mathbf{V} 2$ matches quite nicely between humans and monkeys. The cortical sheet is folded differ- 

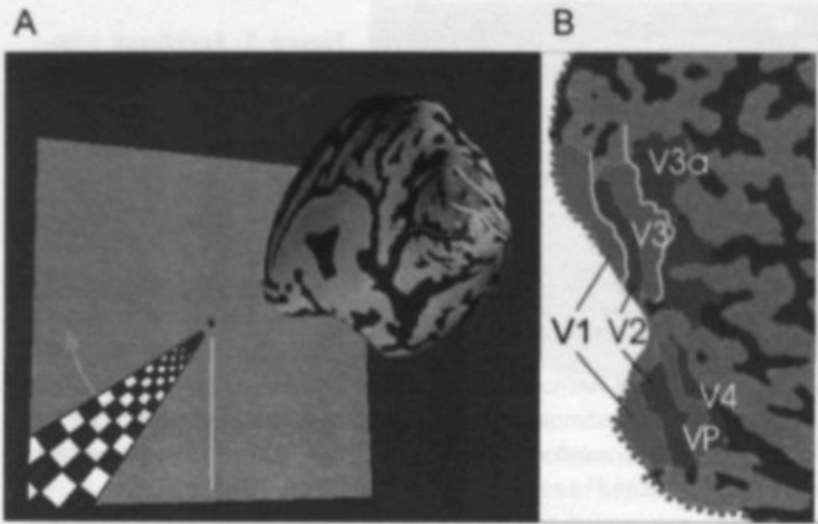

C

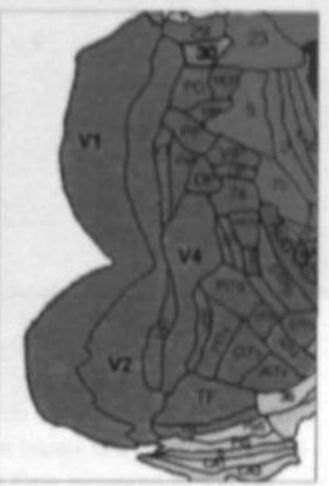

Figure 2. Retinotopic mapping. (A) Visual stimuli in the lower left visual field activate retinotopic visual areas in the right dorsal hemisphere, here shown as an inflated cortical sphere. Convexity coloring (dark) indicates the original sulcus pattern. If the stimulation starts at the lower vertical meridian (yellow line) and rotates clockwise - activity is first seen in right dorsal hemisphere at the V1/N2 and the V3N3a boarder (yellow line on cortex in A and B) and migrates counterclockwise in V1 and V3 (green arrows) and clockwise in V2 and V3a (blue arrows). (B) To flatten the inflated right hemisphere the cortical sphere is cut along the sulcus calcarinus (dotted red line) and medial aspects are turned inside out. Visual areas of normal representation (blue) and mirror image representation (green) are now seen adjacently in the occipital cortex. (C) Comparison to a flattened monkey hemi. sphere atlas taken from Van Essen (http://stp. wustl. edu/; Van Essen et al. 2001). Visual brain areas are labeled and shown in blue.

ently in humans and monkeys and the size differs strongly - comparison of areas would be difficult and misleading if done on grounds of 3D coordinates. Surface based atlases provide a promising alternative for comparing individually inflated and flattened hemispheres (VanEssen et al. 2001, Muckli et al. 2001).

\section{Lower tier visual areas}

$\mathrm{V} 1$ is endowed with a rich columnar structure that arranges maps of preferences for eyes (ocular-dominance columns), orientation (orientation-preference columns), direction and several other features in a complex manner (Swindale, 2000). V2 is organized in a comparable fashion but comprises another compartmentalization of thin and thick stripes that involve different myelination which is leading to different transmission speeds and different functional specialization (see below). From this rich structure human fMRI can only detect the biggest map given by the ocular dominance columns in V1 (Menon et al., 1997). In cats, MMRI has been used to show much smaller columns (orientation) at high magnetic field strength (Kim et al., 2000), but for humans this resolution remains unaccomplished.

Response properties often change gradually between $V_{1}$ and $V_{2}$. For example V1 prefers higher spatial frequencies (Singh et al., 2000) than V2. Illusory contours activate V2 and other higher tier visual areas more than V1 (Goebel et al., 1998a, Mendola et al., 1999). Another clear difference has been shown by modulation of contrast which monotonically 


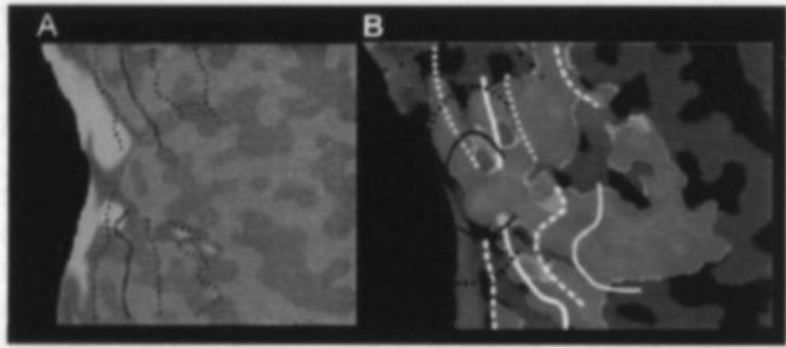

Figure 3. Functional properties of area V1. (A, adapted from Tootell et al. 1998) Response increase to high luminance contrast gratings as compared to low luminance contrast gratings. Big increase is seen in V1 but not in higher visual areas which are less

susceptible to contrast increase. (B) response to monocular stimulation of the amblyopic eye and non-amblyopic eye. Activity that is higher while stimulated through the amblyopic eye is indicated in red. Activity that is higher during normal eye stimulation is seen in green and blue. Lines in ( $A$ and $\mathbf{B}$ ) indicate boarders of visual areas (V1, V2, V3d/P as in Fig 2).

increases in V1 and quickly saturates in higher tier visual areas (Tootell et al., 1998, Fig 3 A).

If, early in life, the visual cortex is stimulated inadequately by both eyes (misalignment of the visual axes - strabismus, or unequal refractory power - anisometropia) the visual system can engage in interocular suppression leading to reduced visual acuity in one eye that is otherwise healthy and property corrected (amblyopia). This interocular suppression is taking effect after area V1. As can be seen in Fig 3 (b) the suppressed -amblyopic eye can activate V1 in some cases even stronger than the normal eye (Muckli et al. 1998b). Higher visual areas after V1, however, are less activated. This attenuation has also been observed in anesthetized amblyopic cats (Schroder et al., 2002).

\section{Higher tier visual areas}

As mentioned above anatomical and functional properties of V1 and V2 match those of monkeys (Foster et al., 1985; Tootell et al. 1998). Higher tier areas beyond V2 however differ in anatomical respect (Van Essen et al., 2001. Fig. 2 C) and functional properties. Area $\sqrt{3}$ in humans is less motion sensitive than those of monkeys, area V3a in contrast is more motion sensitive than $\mathrm{V} 3 \mathrm{a}$ in monkeys (Tootell et al., 1997).

In higher tier visual areas the retinotopic structure dissolves. An example is given in Fig. 1 (right): Early visual areas process the information strictly in the contralateral visual field. In higher tier areas visual processing is responding more and more to both hemifields (equal activation is indicated by yellow color and can be seen on both hemispheres). At this level functional specificy becomes essential for classifying new visual areas. Functional specialization in ventral visual areas can be seen for object categories like faces (Puce et al., 1996; Kanwisher et al., 1997) and places (PPA Epstein et al., 1999), and in dorsal visual areas for spatial transformation (Goebel et al., 1998b) and motion processing (Goebel et al., 1998a). The human motion complex for example can be mapped by contrasting activity to static dots against activity to moving dots (Tootell et al., 1995). It is very likely that the human motion complex contains more areas than just the human homologue to monkey motion selective area MT. Adjacent visual areas MST, MSTd, and FST are selective to higher order motion in the monkey but have not yet been separated reliably in humans. Functional specialization to rotatory motion has been used to tentatively 


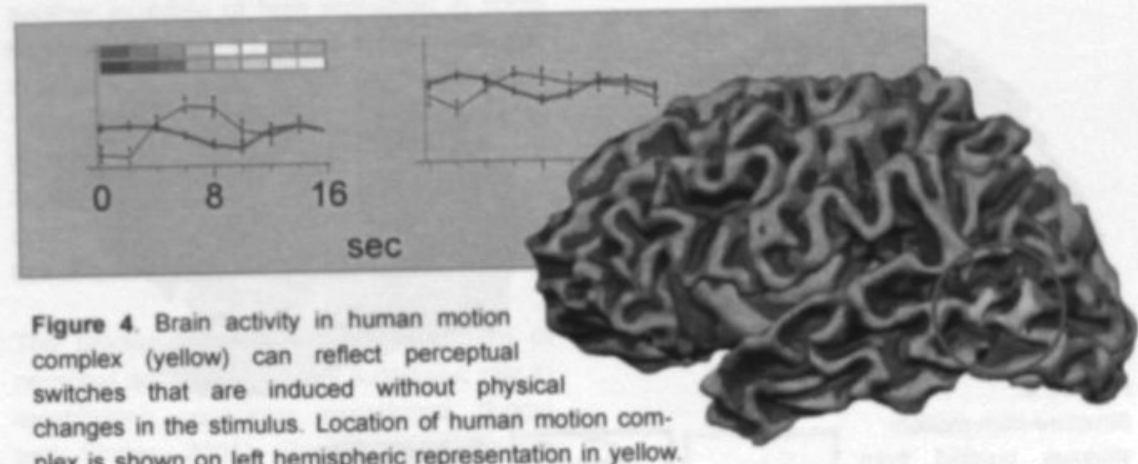
plex is shown on left hemispheric representation in yellow. The BOLD-time-course shown above for left and right motion-complex is time triggered to the perceptual switches indicated by the subjects with button presses. Colored bar indicate the average duration of the percepts. Adaptation to apparent motion stimulus can lead to phases in which blinking is perceived instead of motion. Subsequently activity decreases (blue line after a perceptuai switch) in hMT+. After a short duration the percept of apparent motion comes back again and with the expected delay of several seconds, the BOLD signal from hMT+ rises again (red line; Muckli et al. 2002).

separate human MST from within the human motion complex (Muckli et al., 1998a). Retinotopic mapping of the peripheral representation has been utilized in another approach (Khan et al., 1999).

Functional specialization can be sensitive to the subjective content of ambiguous figures. In studies comprising motion perception, or motion imagery, experiments have been conducted in the absence of changes to the stimulus purely on internal switches (Goebel et al. 1998, Muckli et al. 2002). In Fig. 4 an example is shown for one subject seeing a bistable stimulus that appears to be moving for several seconds until phases occur in which blinking is perceived instead of motion. Perceptual switches occur spontaneously and subjectively. Switches between perceptual states are faithfully reflected in activity changes of area hMT+N5.

\section{Functionally specialized path- ways}

Functional specialization is organized along two separate pathways that have been named after the anatomical location ventrolateral- and dorso-parietal-pathway, or after their most conspicuous functions what- and where pathway (Ungerleider and Mishkin, 1982), or after their assigned task for the visual system, pathway for perception and pathway for action (Milner and Goodale, 1993). Originally different pathways were proposed on grounds of lesion studies, anatomical connections and electrical recordings of many cells in different regions gathered over different experiments on several animals (Ungerleider and Mishkin, 1982; Mishkin et al. 1983). In Fig. 5 functional specialization of the pathways is demonstrated in a human (left) and a monkey (right). Motion specific regions along the dorsal-pathway and face selective areas in the ventral-pathway are shown. A specially designed stimulus was taken to examine how these separate path- 

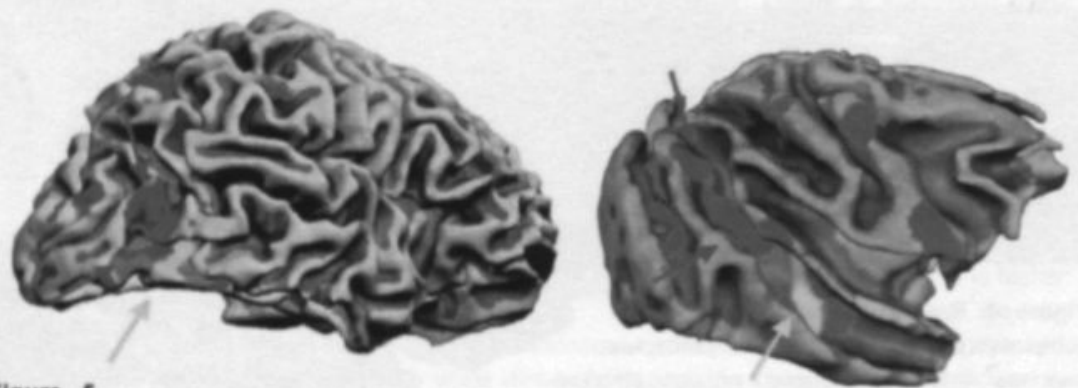

Figure 5.

Structure-from-motionstimulus created from transparent dot displays (green). Each dot corresponds to a point on the surface of a rotating head. Subjects perceive
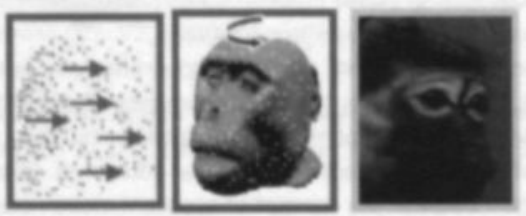

the rotating head as long as the dots are moving consistently. Specialization of brain activation is shown separately for humans (left) and monkeys (right). Red regions correspond to strongest activity during motion. Green regions show areas that are more activated during 3D-structurefrom-motion as compared to simple motion. The green region overlaps with regions specialized for face recognition (yellow). Motion vectors are analyzed preferentially in the where-pathway (red) and faces in the what-pathway (yellow). For the detection and recognition of heads-defined-bymotion (green) both specialized pathways are engaged.

ways cooperate. Moving dots induce the vivid impression of a rotating head which can only be perceived as long as the dots are in motion (Naumer et al., 2000; Muckli et al., 2000; Goebel et al., 2000). Such stimuli are called structure-from-motion and are especially well suited to investigate interactions between motion processing and object recognition (Goebel et al., 1997).

\section{Brain activation and conscious- ness}

So far brain activation measured with fMRI has been shown to reflect functional specificy that is consciously perceived. However this is not always the case. Coming back to the blindsight subjects introduced above some striking observation can be made. The ven- tral and the dorsal visual pathway can be stimulated selectively with visual objects and motion stimuli although the subjects perceive themselves as blind in the respective part of the visual field. Some feature that is not captured by brain imaging is missing to explain why this selective activation in specialized brain region is not resulting in a conscious percept. In our interpretation, which is defended elsewhere (Goebel et al., 2001). the brain activation found in the ventral and dorsal regions ipsilateral to the lesion is isolated from other visual processing regions and looses therefore its effectiveness. It is therefore not the activation above a certain threshold that will allow conscious experience (Paimer 1999) but recurrent loops of neuronal activity between higher and early visual areas (Engel and Singer, 2001). 
The study of amblyopic subjects provides another example of high activation in some brain region, here $\mathrm{V} 1$, which stays ineffective in projecting its activity forward (Muckli et al., 1998b). In cats this neuronal incapacity (Schroder et al., 2002) has been related to a lack of temporal coordination (i.e. reduced synchronization) between neuronal responses (Roelfsema et al., 1994).

The precise temporal response pattern of neuronal activity might play a decisive role in establishing conscious experience (Engel and Singer, 2001). Recent developments have shown that EEG and MEG are capable to find induced synchrony of neuronal responses that can reflect perception of illusory contours or short-term-memory of visual content (Tallon-Baudry et al., 1997; TallonBaudry et al., 1999; Wailz, 2001). A combination of both techniques should be highly complementary, since MEG has an excellent temporal and fMRI a superior spatial resolution (Scherg et al., 1999).

\section{Conclusion}

Brain imaging techniques have shown similarities but also divergence in the arrangement and hierarchy of human visual brain areas as compared to monkey's visual brain areas. Brain imaging techniques have been used to detect activation changes directly linked to subjective changes of perception an approach that is very difficult to achieve in trained monkeys (Leopold and Logothetis, 1996; Dodd et al., 2001). Simultaneous imaging of the whole brain allows to highlight func. tionally specialized pathways and their interaction - without fMRI these comparisons are restricted to pre-selected areas. 


\section{References}

Brodmann K (1905) Beitrage zur historischen Lokalisation der Großhirnrinde. Dritte Mitteilung. Die Rindenfeider der niederen Affen. Journal der Psychologie und Neurologie 4: 177-226.

Chen W, Zhu XH, Thulborn KR, Ugurbil K (1999) Retinotopic mapping of lateral geniculate nucleus in humans using functional magnetic resonance imaging. Proc Natl Acad Sci U S A 96: 2430-2434.

Dodd JV, Krug K, Cumming BG, Parker AJ (2001) Perceptually bistable three-dimensional figures evoke high choice probabilities in cortical area mt. J Neurosci 21: 4809-4821.

Engel AK, Singer W (2001) Temporal binding and the neural correlates of sensory awareness. Trends Cogn Sci 5: 16-25.

Epstein R, Harris A, Stanley D, Kanwisher N (1999) The parahippocampal place area: recognition, navigation, or encoding? Neuron 23: 115 125.

Foster KH, Gaska JP, Nagler M, Pollen DA (1985) Spatial and temporal frequency selectivity of neurones in visual cortical areas V1 and V2 of the macaque monkey. J Physiol 365: 331-363.

Gennari F (1782) Francisci Gennari Parmensis Medicinae Doctoris Collegiati de Peculiari Structura Cerebri Nonnullisque Eius Morbis-Paucae Aliae Anatom Observat Accedunt. Parma, Italy: Regio Typographeo.

Goebel, R., Khorram-Sefat, D. Hacker, H., and Singer, W. Going beyond the information given: the neuronal substrate of phi-motion and shapefrom-motion as revealed by functional magnetic resonance imaging. Neurolmage . 1997.

Goebel R, Khorram-Sefat D, Muckli L, Hacker H, Singer W (1998a) The constructive nature of vision: direct evidence from functional magnetic resonance imaging studies of apparent motion and motion imagery. Eur $J$ Neurosci 10: 1563 1573.

Goebel R, Linden DEJ, Lanfermann $\mathrm{H}$, Zanella FE, Singer W (1998b) Functional imaging of mirror and inverse reading reveals separate coact. vated networks for oculomotion and spatial transformations. Neuroreport 9: 713-719.
Goebel R, Muckli L, Zanella FE, Singer W, Stoerig P (2001) Sustained extrastriate cortical activation without visual awareness revealed by fMRI studies of hemianopic patients. Vision Res 41: 1459. 1474.

Goebel, R., Naumer, M., Muckli, L., van Meer, H., Engel, A. K., Zanella, F. E., and Singer, W. Structure-from-motion of three dimensional objects differentially activates specialized areas in the ventral processing stream. Soc.Neurosci.Abstr. 26[2], 1584. 2000.

Kanwisher N, McDermott J, Chun MM (1997) The fusiform face area: a module in human extrastriate cortex specialized for face perception. J Neurosci 17: 4302-4311.

Khan, R. M., dougherty, R. F., Wandell, B. A., Newsome, W. T., and Heeger, D. J. Functionally distinct motion areas in human visual cortex. Soc. Neurosci.Abstr. 25[1], 275. 1999.

Kim DS, Duong TQ, Kim SG (2000) High-resolution mapping of iso-orientation columns by MRI. Nat Neurosci 3: 164-169.

Leopold DA, Logothetis NK (1996) Activity changes in early visual cortex reflect monkeys' percepts during binocular rivaliry. Nature 379: 549 553.

Mendola JD, Dale AM, Fischl B, Liu AK, Tootell RB (1999) The representation of illusory and real contours in human cortical visual areas revealed by functional magnetic resonance imaging. J Neurosci 19: 8560-8572.

Menon RS, Ogawa S, Strupp JP, Ugurbil K (1997) Ocular dominance in human V1 demonstrated by functional magnetic resonance imaging. J Neurophysiol 77: 2780-2787.

Milner AD, Goodale MA (1993) Visual pathways to perception and action. Prog Brain Res 95: 317. 337.

Mishkin M, Ungerleider LG, Macko KA (1983) Object vision and spatial vision. two cortical pathways. Trends Neurosci 6: 414-417.

Muckli, L., Goebel, R., Singer, W., and Munk, M. H. J. Functional MRI of Anesthetized Monikeys using Three dimensional structure from motion stimuli. Soc.Neurosci.Abstr. 26[2], 1583. 2000. 
Muckli L, Kriegeskorte N, Lanferman H, Zanella FE, Singer W, Goebel R (2002) Apparent motion: Event-related MRI of perceptual switches and states. J Neurosci. in press.

Muckli, L., Munk, M. H. J, Staedtler, E., Baron, J., Fiesser, A. and Singer, W. (2001) Functional and structural comparison of human and monkey brains by cortex based morphing. Soc.Neurosci. Abstr.

Muckli, L., Singer, W., Zanella, F. E., and Goebel, R. (1998a) Rotation selective regions in the human motion complex and its satellites. Soc.Neurosci.Abstr. 24[Part 2], 1746.

Muckli, L., Tonhausen, N., Goebel, R., Lanfermann, H., Zanella, F. E., Singer, W. and Sireteanu, R. (1998b). Reduced fMRI signal response to monocular amblyopic eye stimulation in extrastriate visual areas. Neurolmage 7[4], S19.

Naumer, M., Bonte, M., Grether, M., Muckli, L. Engel, A., Zanella, F. E., Singer, W, and Goebel, R. (2000) Three-dimensional structure-frommotion activates shape-specific areas in the ventral visual processing stream. Neurolmage 11[5]. S691.

Puce A, Allison T, Asgari M, Gore JC, McCarthy G (1996) Differential sensitivity of human visual cortex to faces, letterstrings, and textures: a functional magnetic resonance imaging study. J Neurosci 16: 5205-5215.

Roelfsema PR, Konig P, Engel AK, Sireteanu R, Singer W (1994) Reduced synchronization in the visual cortex of cats with strabismic amblyopia. Eur J Neurosci 6: 1645-1655.

Scherg, M., Linden, D. E. J, Muckli, L., Roth, R. Druen, K. Ille, N., Zanella, F. E., Singer, W., and Goebel, R. (1999) Combining MEG with AMRI in studies of the human visual system. Advances in Biomagnetism. Sendai, Tohoku University Press.

Schroder JH, Fries P, Roelfsema PR, Singer W, Engel AK (2002) Ocular dominance in extrastriate cortex of strabismic amblyopic cats. Vision Res 42: 29-39

Sereno MI, Dale AM, Reppas JB, Kwong KK, Belliveau JW, Brady TJ, Rosen BR, Tootell RB (1995) Borders of multiple visual areas in humans revealed by functional magnetic resonance imag. ing. Science 268: 889-893.
Singh KD, Smith AT, Greenlee MW (2000) Spatiotemporal frequency and direction sensitivities of human visual areas measured using MRRI. Neuroimage 12: 550-564.

Swindale NV (2000) How many maps are there in visual cortex? Cereb Cortex 10: 633-643.

Tallon-Baudry C, Bertrand O, Wenbruch C, Ross B. Pantev C (1997) Combined EEG and MEG recordings of visual $40 \mathrm{~Hz}$ responses to illusory triangles in human. Neuroreport 8: 1103-1107.

Tallon-Baudry C, Kreiter A, Bertrand O (1999) Sustained and transient oscillatory responses in the gamma and beta bands in a visual short-term memory task in humans. Vis Neurosci: 449-459.

Tootell RB, Hadjikhani NK, Vanduffel W, Liu AK, Mendola JD, Sereno MI, Dale AM (1998) Functional analysis of primary visual cortex (V1) in humans. Proc Natl Acad Sci U S A 95: 811-817.

Tootell RB, Mendola JD, Hadjikhani NK, Ledden PJ, Liu AK, Reppas JB, Sereno MI, Dale AM (1997) Functional analysis of V $3 A$ and related areas in human visual cortex. J Neurosci 17: 7060-7078.

Tootell RB, Reppas JB, Kwong KK, Malach R, Born RT, Brady TJ, Rosen BR, Belliveau JW (1995) Functional analysis of human MT and related visual cortical areas using magnetic resonance imaging. J Neurosci 15: 3215-3230.

Ungerleider LG, Mishkin M (1982) Two cortical visual systems. In: Analysis of visual behaviour (Ingle DJ, Goodale MA, Mansfield RJW, eds), pp 549-586. Cambridge: MIT Press.

Van Essen DC, Lewis JW, Drury HA, Hadjikhani N. Tootell RB, Bakircioglu M, Miller MI (2001) Mapping visual cortex in monkeys and humans using surface-based atlases. Vision Res 41: 1359-1378

Waltz JA, Linden DEJ, Muckdi L, Witrowski A, Prvulovic D, Goebel R, Singer W, Munk MHJ. Correspondence between EEG band power and BOLD signal in humans performing a visual short term memory task. Cogn Neurosci.Abstr. 2001

Weiskrantz L., Warrington EK, Sanders MD, Marshall J (1974) Visual capacity in the hemianopic field following a restricted occipital ablation. Brain 97: 709-728. 


\section{Chapter 2}

\section{Cerebral correlates of impaired grating perception in human amblyopia}

This chapter corresponds to:

Lars Muckli ${ }^{1}$, Ruxandra Sireteanu ${ }^{2}$, Nathalie Tonhausen ${ }^{2}$, Stefan KieB ${ }^{1}$, Heinrich Lanfermann ${ }^{3}$, Wolf Singer ${ }^{1}$ and Rainer Goebel ${ }^{4}$ (2002) Cerebral correlates of impared grating perception in human amblyopia. (submitted)

${ }^{1}$ Max Planck Institute for Brain Research, Department of Neurophysiology, Frankfurt a. M.. Germany. ${ }^{2}$ Department Physiological Psychology - Biopsychology. Faculty of Psychology ${ }^{3}$ Department of Neuroradiology. Johann Wolfgang Goethe-Universitat, Frankfurt a. M., Germany ${ }^{4}$ Department of Neurocognition, Faculty of Psychology. Maastricht University, Maastricht. Netherlands. 


\section{Abstract}

Amblyopia designates visual deficits that result from abnormalities in the experience dependent development of cortical functions. We investigated in human subjects neuronal correlates of amblyopic deficits resulting from early onset strabismus or anisometropia by monitoring visual responses in early visual areas V1, V2, V3, VP, V3a, V4/N8 with functional magnetic resonance imaging (fMRI). Areas of the visual cortex were identified by mapping retinotopic representations and amblyopic deficits were measured by activating cortex with square wave gratings of four spatial frequencies $(0.5,4.0,8.0$ and $16.0 \mathrm{c} / \mathrm{deg})$. In lower visual areas (V1 / V2) stimuli presented to the normal and the amblyopic eye evoked strong responses irrespective of spatial frequency while responses to the amblyopic eye were reduced in higher areas of the ventral visual stream (V4 / V8; lateral occipital complex, LOC), especially for high frequency gratings. This suggests that transmission of activity from the amblyopic eye is increasingly impaired while it is relayed towards higher processing levels. 


\section{Introduction}

Amblyopia is a developmental deficit caused by inadequate visual stimulation early in life. It is defined clinically by a reduction of visual acuity in otherwise healthy and properly corrected eyes. The most common factors leading to amblyopia are a misalignment of the visual axes of the two eyes (strabismus). unequal refractive power in the two eyes (anisometropia), or a physical obstruction, like a lens opacity (cataract) or a drooping eyelid (ptosis), preventing visual signals from reaching the retina of one or both eyes (visual deprivation). Strabismic subjects use only one of the two eyes at a time for vision in order to avoid double images. If, rather than alternating between the two eyes, they use always the same eye, the dominant eye, the other develops amblyopia because the cortical networks connected to this suppressed eye do not develop normally. In most cases of amblyopia, binocular functions are lost or at least severely reduced and when both eyes are open, signals from the amblyopic eye are usually suppressed and not perceived. When the normal eye is closed subjects report reduced visual acuity and difficulties with figure ground segregation when viewing with the amblyopic eye.

The different ethiologies can lead to different deficits: in anisometropic and deprivation amblyopia reduced acuity is the dominant disturbance. Dark adaptation affects visual function in anisometropic, but not in strabismic amblyopia (Hess and Howell, 1978; Hess et al., 1980). With strabismic amblyopia, by contrast, there may be additional symptoms such as spatial distortions (Lagreze and Sireteanu, 1991), difficulties to separate nearby visual contours (crowding: Hess et al., 2000a; Hess et al., 2000b), and anomalous retinal correspondence (Duke-Elder 1973). Furthermore, the patterns of interocular suppression associated with the two kinds of amblyopia differ (Sireteanu et al., 1981).
Therefore, it has been suggested that strabismic and anisometropic amblyopia form two separate syndromes, possibly involving different neural substrates: while anisometropic amblyopia was believed to be a mild form of deprivation amblyopia, possibly involving a reduction in the activity of single cells in the striate visual cortex (area 17, or V1), strabismic amblyopia was proposed to result from active suppression and to involve different cortical mechanisms. Early studies sug. gested a reduction and scattering of conduction velocities in thalamo-cortical and intracortical transmission (Rauschecker and Singer, 1979; Singer et al., 1980), or spatial scrambling of the cortical representation of the amblyopic eye (Hess and Field, 1994).

Physiological studies in cats rendered amblyopic by defocusing one eye or by inducing strabismus support the suggestion of a functional dichotomy between strabismic and anisometropic amblyopia: in strabismic kittens with a demonstrated loss of function, the frequency of cells driven by the amblyopic eye is only mildly reduced in areas 17; a substantial loss of cells driven by the affected eye occurs only in extrastriate visual areas such as the lateral suprasylvian sulcus area PLLS (Sireteanu and Best, 1992) and area 21a (Schroder et al., 2002). By contrast anisometropic and visually deprived cats show a reduction in the relative frequency of single cells driven by the affected eye already in area 17 (Hubel and Wiesel, 1965; Wiesel and Hubel, 1965; Movshon et al., 1987).

In the monkey, the search for a cortical substrate of strabismic amblyopia has been confined to area V1. Kiorpes, Kiper, O'Keefe, Cavaanagh and Movshon (1998; Kiorpes et al., 1998) found that the two eyes were about equally effective in driving cortical cells but neurons driven by the amblyopic eye had reduced spatial resolution. However, optimal spatial frequency and peak contrast sensitiv. 
ity of these neurons were better than expected from behavioral examination. From this the authors concluded that a full account of the physiological basis of amblyopia would require the investigation of processing stages beyond striate cortex (for a review see Kiorpes and McKee, 1999).

Recent evidence from our laboratory led to the hypothesis that strabismic amblyopia might be associated with a selective loss of response synchronisation among otherwise well responding striate cortex cells driven by the amblyopic eye (Roelfsema et al., 1994). This lack of temporal structure between members of active cell assemblies in primary visual cortex has been proposed to be the cause for the reduced neuronal activity in extrastriate visual regions (Sireteanu and Best, 1992; see also Schroder et al., 2002).

In humans, mechanisms underlying amblyopia have been investigated with psychophysical methods (Sireteanu, 2000), post mortem studies (Horton and Stryker, 1993; Horton and Hocking. 1996), electrophysiological techniques (Levi and Manny 1982), and recently, functional imaging (Goodyear et al. 2000). The results of imaging studies are somewhat contradictory: A study using SPECT reported significant reductions of activity in occipital visual cortex when the amblyopic rather than the normal eye was stimulated with strobe flashes or reversing checkerboards (Kabaskal et al. 1995). In this study it was not possible to delineate the various subdivisions of the visual cortex. Preliminary studies combining magnetoencephalography (MEG) with visual stimulation using gratings of different spatial frequencies suggested a selective loss already in area V1 (Anderson et al. 1999). In contrast, studies with the Positron Emission Tomography (PET) technique suggest that the amblyopic deficit manifests itself only at the level of prestriate areas 18 and 19 (V2 and V3; Imamura et al., 1997). In this study, as in the pre- vious ones, conclusions are based on pooled results from a heterogeneous group of amblyopic subjects, and a precise localization of activity to particular cortical areas was not possible.

Goodyear et al. (2000) used fMRI to analyze responses in V1 to $22 \%$ contrast gratings at various spatial frequencies and found that fewer voxels were activated following stimulation of the amblyopic eye but with unreduced amplitude. This was interpreted as reflection of an elevated contrast threshold but unimpaired contrast sensitivity. The assumption was that the amplitude of activated voxels corresponds to the firing rate of the responsive neurons, and once the detection signal-to-noise ratio of neural activity has been surpassed, the perception of contrast depends on the average firing rate of the responsive neurons. Again no attempts had been made to identify the borders between visual areas by retinotopic mapping nor have any extrastriate regions been considered in the analysis.

Here we applied fMRI to identify visual areas with a retinotopic mapping procedure and to investigate the sites of activation deficits in strabismic and anisometropic amblyopes that have been characterized by prior orthoptic and psychophysical tests. We used high frequency gratings that induce perceptual deficits for the amblyopic eye and related the fMRI signal of retinotopic visual areas V1, V2, V3, VP, V3a, V4/V8 and LO to these deficits.

Preliminary data of this study were published in abstract form (Sireteanu et al.1998; Muckli et al. 1998).

\section{Methods}

\section{Subjects}

The study was performed with 4 anisometropic amblyopes (2 females, 2 males), 4 


\begin{tabular}{|c|c|c|c|c|c|c|c|c|c|c|c|}
\hline \multirow{3}{*}{$\beta$} & \multirow{3}{*}{ Subj } & \multirow{3}{*}{$\frac{\left\{\begin{array}{l}\text { sex } / \\
\text { age }\end{array}\right.}{f / 25}$} & \multirow{2}{*}{ Eye } & \multicolumn{2}{|c|}{$\begin{array}{l}\text { Refraction } \\
\text { sph - cyl }\end{array}$} & \multirow{2}{*}{\begin{tabular}{|c|} 
visus \\
1.25 \\
$\ldots \ldots .$.
\end{tabular}} & \multirow{3}{*}{\begin{tabular}{|c|}
$\begin{array}{c}\text { fixation } \\
\text { f: foveolar }\end{array}$ \\
$f$ \\
$7-8$ nasal
\end{tabular}} & \multicolumn{2}{|c|}{$\begin{array}{c}\text { strabismus } \\
\text { far / near }\end{array}$} & \multicolumn{2}{|r|}{ observations } \\
\hline & & & & -3.25 & $0.25 / 30^{\circ}$ & & & \multirow[t]{2}{*}{$+12^{*}$} & \multirow[t]{2}{*}{$+15^{\circ}$} & \multirow[t]{2}{*}{ are } & \multirow{2}{*}{$\begin{array}{c}\text { squint onset } 2-3 \text { years, no family } \\
\text { history, no surgery, first RX } 4 \\
\text { years }\end{array}$} \\
\hline & & & $L$ & -3.25 & $\cdot$ & 0.20 & & & & & \\
\hline & \multirow{2}{*}{ KSM } & \multirow{2}{*}{$f / 49$} & $\boldsymbol{R}$ & -0.25 & - & 0.40 & $3^{*}$ nasal & \multirow{2}{*}{$+3^{\circ}$} & \multirow{2}{*}{$+3^{\circ}$} & \multirow{2}{*}{ hare } & \multirow{2}{*}{$\begin{array}{l}\text { family history, no surgery, } \\
\text { microstra-bismus with identity }\end{array}$} \\
\hline & & & $\mathrm{L}$ & & $-0.25 / 0^{\circ}$ & 1.00 & $f$ & & & & \\
\hline \multirow[t]{2}{*}{$s$} & \multirow{2}{*}{ PZ } & \multirow{2}{*}{$\mathrm{m} / 36$} & $R$ & +1.75 & - & 1.00 & $f$ & \multirow{2}{*}{$+10^{\circ}$} & \multirow{2}{*}{$+12^{*}$} & \multirow{2}{*}{ nhare } & \multirow{2}{*}{$\begin{array}{l}\text { family history, no surgery. } \\
\text { occlusion therapy }\end{array}$} \\
\hline & & & $i$ & +3.00 & - & 0.40 & $i$ & & & & \\
\hline \multirow[t]{2}{*}{5} & \multirow[t]{2}{*}{ RS } & \multirow[t]{2}{*}{$\mathrm{m} / 29$} & $R$ & +3.50 & $10 / 150^{\circ}$ & 0.30 & (f) unstable & \multirow[t]{2}{*}{$+1^{\circ}$} & \multirow{2}{*}{$+2^{\circ}$} & \multirow{2}{*}{ harc } & \multirow{2}{*}{$\begin{array}{c}\text { no family history, no surpery. } \\
\text { occlusion therapy } 6 \text { years. } \\
\text { microstrabismus }\end{array}$} \\
\hline & & & $\mathrm{L}$ & +2.25 & - & 1.60 & $f$ & & & & \\
\hline \multirow{2}{*}{ 夏 } & EM & $f / 44$ & $\mathbf{R}$ & $+0,25$ & $0,50 / 0^{\circ}$ & 1.40 & $f$ & non & & noc & family history, no surgery, first $R X$ \\
\hline & & 1174 & $L$ & +3.25 & $1.25 / 160^{\circ}$ & 0.50 & $f$ & non & & nic & \\
\hline & $\mathrm{MaM}$ & $f / 67$ & $R$ & -7.00 & $2.00 / 170^{\circ}$ & 0.08 & $f$ & \begin{tabular}{|l|}
$-1^{\circ}$ \\
$+\mathrm{VD}$
\end{tabular} & & . & no surgery, no therapy. first RX 50 \\
\hline & & & $\mathrm{L}$ & +1.50 & - & 1.00 & $f$ & $5^{\circ}$ & + VD $5^{\circ}$ & ( & \\
\hline 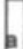 & MM & $\mathrm{m} / 24$ & $\boldsymbol{R}$ & +3.00 & $1.25 / 145^{\circ}$ & 0.60 & $f$ & $-12^{\circ}$ & $-10^{\circ}$ & $\operatorname{arc} 7^{\circ}$ & Squint onset $3-4$ years, family \\
\hline & $\min$ & $m / 24$ & $\mathrm{i}$ & +0.75 & $1.75 / 0^{\circ}$ & 1.25 & i & $|-12|$ & & nasal & therapy \\
\hline 旦 & RK & $m / 34$ & $\mathbf{R}$ & $+3,50$ & & 1.25 & $f$ & none & $+1^{\circ}$ & nre & family history, no surgery, first $R X$ \\
\hline & & & $L$ & $+6,00$ & & 0.80 & $f$ & & & & \\
\hline
\end{tabular}

Table 1. Orthoptic data of amblyopic subjects particpating in the fMRI studies. s.strabismic; a: anisometropic; L:left eye; R: right eye; sph: dioptre sphere; cyl: cylindrical lenses

strabismic amblyopes (3 females, 3 males) and 4 normally-sighted, emmetropic subjects ( 3 females, one male) matched for age with the experimental group. All subjects underwent orthoptic examination by a professional orthoptist. Tests included: objective refraction; Snellen visus for near and far; fixation pattern; squint angle; stereopsis; retinal correspondence and clinical history. The subjects were classified as being primarily anisometropic if they had a refractive difference between the two eyes of at least $2 \mathrm{D}$ spherical equivalent. To be included in the experimental group. subjects were required to have an amblyopic deficit of at least two lines on a Snellen chart test. Orthoptic details of the amblyopic subjects are shown in Tab 1.

Prior to psychophysical testing, the subjects' contrast sensitivity was tested for near and far, using the Vistech charts. Written informed consent was obtained from all subjects prior to the study after the procedure was fully explained.

\section{MRI Data Acquisition}

Functional magnetic resonance imaging was performed at $1.5 \mathrm{~T}$ (Siemens Magnetom Vision, with a gradient overdrive) using the standard head coil and a gradient echo EPI sequence ( 11 slices, $T E=69 \mathrm{~ms}, T R=3000$ $\mathrm{ms} ; \mathrm{FA}=90^{\circ}, \mathrm{FOV}=200 \times 200 \mathrm{~mm}^{2}$, voxel size $1.6 \times 1.6 \times 3-4 \mathrm{~mm}^{3}$ ). For binocular mapping of area boundaries an alternative sequence was used $(16$ slices, $T R=2000$ $\mathrm{ms} ; \mathrm{FA}=90^{\circ}$, voxel $3.2 \times 3.2 \times 4 \mathrm{~mm}^{\circ}$ ). A T1 . weighted $3 D$ magnetization-prepared rapid acquisition gradient echo sequence (MP. RAGE, $T R=9.7, T E=4 \mathrm{~ms}, F A=12^{\circ}$ ) lasting 
8 minutes was recorded in the same session as the functional measurements for later matching of anatomical coordinates (voxel size $1.0 \times 1.0 \times 1.0 \mathrm{~mm}^{3}$ ). An additional T1weighted $3 \mathrm{D}$ data set, tuned to optimise the contrast between grey versus white matter, was recorded in a separate recording session lasting 24 minutes (fast low angle shot [FLASH] sequence), and was used for high resolution 3D reconstruction. Visual stimuli were delivered under computer control (Digital DECpc Celebris XL 590 or DELL Inspiron 7500 ) via a LCD projector (EIKI LC-6000 or Sony VPL XP 20). Visual stimuli were generated using the Microsoft DirectX graphics library. The image was back-projected onto a screen positioned at the foot end of the scanner.

\section{Eye separation}

To ensure that during fMRI experiments the two eyes could be stimulated separately, subjects wore custom-made light-tight red-green goggles with carefully matched luminance transmittances and stimuli were presented in the corresponding colors. To control for possible individual variance in spectral sensitivity. pretests were performed with four pairs of goggles: red-green (red in front of the dominant eye), green-red (green in front of the dominant eye), red-red, and green-green. Filters made of the same material as the goggles were flipped in alternation in front of the projector to limit vision for one eye. Balance of luminance was obtained by use of additional neutral density filters.

\section{Psychophysics}

The psychophysical tests consisted of monocular and binocular measurements of grating acuity. Measured variables were detection threshold and response latencies. Stimuli consisted of high-contrast (98\%) square-wave gratings of different spatial fre- quencies (0.5 - 36 cycles / degree [cpd]). All gratings were presented through a round aperture of 9 deg diameter on a computer screen in $205 \mathrm{~cm}$ distance. Gratings of different spatial frequencies were presented to the subject in random order and in different orientations (vertical or horizontal) and interleaved with blank screens. The task of the subject was to press one of two buttons of the computer mouse to indicate the orientation of the grating. Half of the subjects used the index finger to indicate vertical orientation and the middle finger to indicate horizontal orientation of the grating: for the other half of the subjects, the finger coding was reversed. When no grating was visible, the subject was asked to press a random button (forcedchoice procedure). Each subject was presented with gratings of at least six different spatial frequencies. To assure that at least two gratings were subthreshold and two suprathreshold, the range of spatial frequencies was adjusted for each subject and each eye. About 40 assessments were made for each spatial frequency. Each subject was tested wearing his/her prescribed spectacles for corrections of refrective errors. Each subject participated in two experimental sessions (a binocular and a monocular session). In each session, all possible combinations of goggles were used (red-green and green-red for the monocular measurements; red-red and green-green for the binocular measurements).

\section{Imaging experiments}

Each subject participated in at least two fMRI sessions, each of them including the monocular mapping of retinotopic coordinates (polar angle and eccentricity. see below). a threedimensional anatomical mapping and the assessment of monocular response sensitivity to gratings of four different spatial frequencies $(0.5,4.0,8.0$ and $16.0 \mathrm{cpd})$. To achieve monocular stimulation, the subjects wore one 
of the red-green goggles, and red or green filters with matched transmittances were placed in front of the projector. Red-green and green-red goggles were switched between sessions. Gratings of a given spatial frequency were presented for periods of 24 seconds and alternated between horizontal and vertical every 2 seconds. Each stimulus sequence was followed by 24 seconds of fixation, after which the other eye was stimulated with a grating of the same spatial frequency. The dominant eye was always stimulated before the non-dominant eye. Gratings could be presented either in ascending or in descending order of spatial frequency. Each eye was stimulated at least twice at a particular spatial frequency. Stimuli were back-projected onto a screen positioned at the foot end of the scanner and spanned maximally $15 \times 20$ deg.

Phase encoded retinotopic mapping was assessed in each subject and included mapping of eccentricity and polar angle (Sereno et al., 1995: Goebel et al., 1998a; Linden et al., 1999; Goebel et al., 2001). In the eccentricity mapping experiment, black and white checkerboard patterns were presented in a ring-shaped configuration and were flickered at a rate of $4 \mathrm{~Hz}$. The ring started with a radius of $1^{\circ}$ and slowly expanded to a radius of $12^{\circ}$ within 96 seconds. In the polar angle mapping experiment, the checkerboard pattern consisted of a ray-shaped disk segment subtending $22.5^{\circ}$ of polar angle. The ray started at the left horizontal meridian and slowly rotated clockwise for a full cycle of $360^{\circ}$ within $96 \mathrm{sec}$. Each mapping experiment consisted of four repetitions of a full expansion or rotation, respectively. Each subject participated in two monocular mapping sessions. Four subjects (EM, RK, KSM, PZ) participated additionally in a binocular mapping session. Binocular mapping included 10 repetitions of the rotating ray-shaped stimulus and 7 repetitions of the expanding ring. each cycle lasting for $64 \mathrm{sec}$.

\section{Data analysis}

Data were analysed with BrainVoyager 2000 (BrainInnovation, Maastricht. Netherlands: Goebel et al., 1998) and included removal of low-frequency drifts, 3D motion detection and correction, determination of Talairach coordinates. multiple regression analysis, cortex reconstruction, inflation and flattening. For the statistical analysis of retinotopic mapping experiments, parametric and non-parametric cross-correlation analysis was applied (Linden et al., 1999). Sites activated at particular eccentricities and polar angles were identified through selecting the lag value that resulted in the highest cross-correlation value for a particular voxel. The obtained lag values at particular voxels were encoded in pseudocolour on corresponding surface patches (triangles) of the reconstructed cortical sheet.

In the group analysis pixels were included into the statistical map if the obtained multiple correlation value was significant at an alpha level of 0.05 . P-values were corrected for multiple comparison using cortex-based Bonferroni adjustment, i.e. the number of voxels included for correction were limited to gray matter voxels (38400).

Retinotopic mapping experiments were analysed with a lowered threshold $r>0.14$ and were restricted to the occipital cortex. Individual analysis followed the retinotopic mapping and was restricted to the functionally defined borders of visual areas for the ROI-statistics (see below) or to the occipital cortex for the analysis of contrasts

Based on the polar angle mapping experiment. the boundaries of retinotopic cortical areas V1, V2, V3, VP, V3A and V4V were estimated on the flattened cortical surface (see below).

The main experiment was analysed with a multiple regression model consisting of four 
predictors for dominant- (d-) and amblyopic(a-) eye stimulation at low ( $\mathrm{I}-0.5$ and $4 \mathrm{cpd}$ ) and high (h- 8 and $16 \mathrm{cpd}$ ) spatial frequency (thus: dl, al, dh, ah). The overall model fit was assessed using a $F$-statistic. The relative contribution of two of the four predictors RC = $(\mathrm{b} 1$ - b2) $/(\mathrm{b} 1$ + b2) was visualised with a redyellow-blue-green pseudo-colour scale (b1 and b2 corresponding to dl and al or to dh and ah). The middle values yellow and blue correspond to minimal differences. Contrast maps were used for the visualisation of individual results. Contrast maps ignore regions with identical contribution of b1 and b2 and indicate higher activity in pseudo-colour scale pink-red for higher activity during amblyopic eye stimulation or green-blue for higher activity during dominant eye stimulation, where pink and blue indicate only a slight tendency of higher activity $(p<0.20)$ and red and blue a significantly higher activity $(p<0.05)$.

Statistical maps were superimposed onto the original functional scans and incorporated into the high-resolution 3D MRI data sets through interpolation to the same resolution (voxel size: $1.0 \times 1.0 \times 1.0 \mathrm{~mm}$ ). This allowed us to produce 3D-reconstructions of the brain with superimposed 30 statistical maps. Since the 20 functional and 30 structural measurements were performed within the same recording session, coregistration of the respective data sets could be computed directly based on the Siemens slice position parameters of the $12^{*}$-weighted measurement (number of slices, slice thickness, distance factor, Tra-Cor angle, FOV, shift mean, off-center read, off-center phase, in plane resolution) and the T1-weighted 30 MP RAGE measurement (number of sagittal partitions, shift mean, off-center read, off-center phase, resolution). In order to compare actvated brain regions across sessions, anatomical and functional 30 data sets were transformed into Talairach space. Results were visualized by superimposing 3D statistical maps on reconstructions of the cortical sheet.

The recorded high resolution T1-weighted 3D recordings were used for surface reconstruction of both cortical hemispheres of each subject (for details Kriegeskorte and Goebel, 2001). Results of the group analysis were presented on inflated surface reconstructions of the MNI-template brain (courtesy of the Montreal Neurological Institute). The white/ grey matter border was segmented with a region-growing method preceded by inhomogeneity correction of signal intensity across space. The borders of the two resulting segmented subvolumes were tessellated to produce a surface reconstruction of each cortical hemisphere. The tessellation of the white/ grey matter boundary of a single hemisphere typically consists of approximately 250,000 triangles. An iterative 30 morphing algorithm was used to let the surface grow smoothly into the grey matter. Through visual inspection, this process was halted when the surface reached the middle of grey matter corresponding approximately to layer 4 of the cortex. The resulting surface was used as the reference mesh for projecting functional data on folded, inflated or flattened representations. A morphed surface has always a link to the folded reference mesh so that functional data can be shown at the correct location of an inflated as well as flattened representation. This link was also used to keep geometric distortions to a minimum during inflation and flattening through inclusion of a morphing force that keeps the distance between vertices and the area of each triangle of the morphed surface as close as possible to the respective values of the folded reference mesh. 


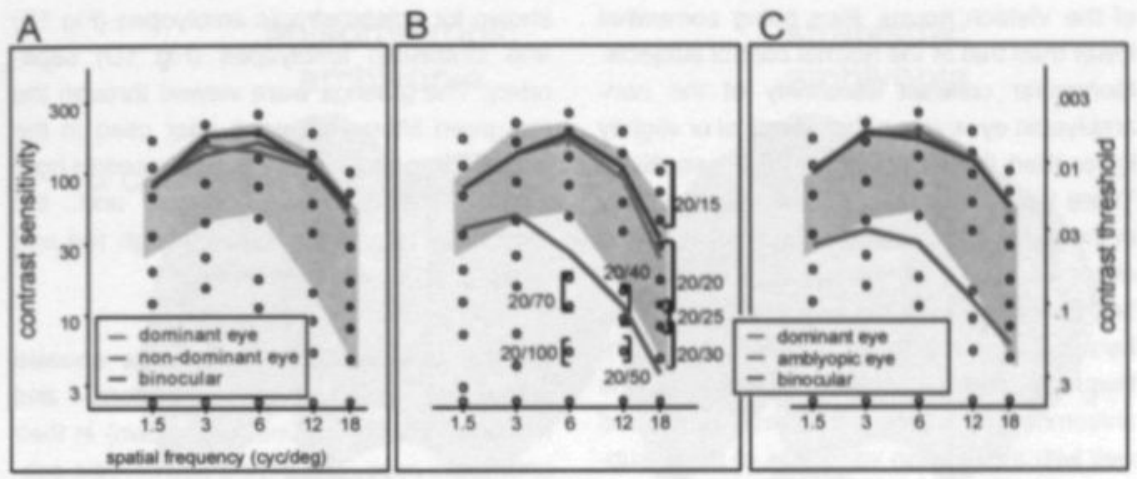

D

$E$

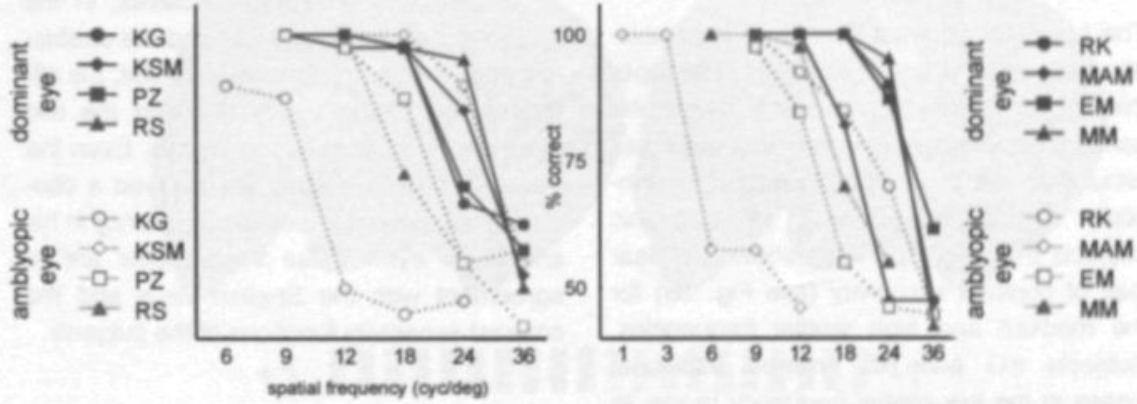

Figure 1. Contrast sensitivity and psychometric functions. Monocular and binocular contrast sensifivity at different spatial frequencies averaged for control subjects (A), for strabismic amblyopes (B) and for anisometropic amblyopes (C). Contrast sensitivity functions were acquired using Vistech charts. Normal contrast sensitivity as provided by the Vistech norms is indicated by gray shading. Corresponding Snellen acuity is exemplified with numbers in (B). Amblyopic subjects show clear deficit with their amblyopic eye (B, D). Contrast sensitivity for the dominant eyes and for binocular vision are comparable to age matched controls (A). Individual performance of orientation discriminafion experiment for the group of strabismic amblyopia (D) and anisometropic amblyopia (E). Percent correct responses are plotted as a function of spatial frequencies for the monocular conditions (red dashed: amblyopic eyes; green lines dominant eyes).

\section{Results}

\section{Psychophysics}

\section{Contrast sensitivity}

The averaged binocular and monocular contrast sensitivity of the control subjects and the two groups of amblyopic subjects is shown in Fig 1A-C. For the control subjects (Fig. 1A) the monocular values were equal in the two eyes and, for the younger subjects LC, NT and TS, they tended to fall in the upper range or slightly above the upper limits of the Vistech norms. For the elderly subject EB, the monocular contrast sensitivity values fell within the Vistech norms. Binocular values were equal to the monocular values.

In anisometropic amblyopes (Fig. 1C), binocular contrast sensitivity fell in the upper range 
of the Vistech norms, thus being somewhat lower than that of the normal control subjects. Monocular contrast sensitivity of the nonamblyopic eyes was either identical or slightly lower than the binocular contrast sensitivity. There was a clear loss of contrast sensitivity in the amblyopic eyes (see Fig. 1B), involving either all spatial frequencies (subjects MaM and EM) or only the medium and high spatial frequencies (subjects MM and RK). The highfrequency loss in contrast sensitivity in the anisometropic amblyopic subjects correlated well with the Snellen visus loss of these subjects (see Table 1).

The binocular contrast sensitivity in strabismic amblyopes (Fig. 1B) also fell in the upper third of the Vistech norms. The monocular contrast sensitivity of the dominant eyes was either identical or slightly lower than the binocular contrast sensitivity. In all strabismic subjects the amblyopic eyes showed a clear loss of contrast sensitivity (see Fig. 18) for the medium and high spatial frequencies. Subjects KG and RS showed additional losses in the low spatial frequency range. In subjects PZ and KSM, the low spatial frequencies were relatively spared. The high spatial frequency losses in the amblyopic eyes correlated well with the losses obtained using Snellen visus charts (see Table 1).

One of the strabismic subjects $(\mathrm{MH})$ had a history of amblyopia but now used both eyes in alternation for fixation. Her monocular contrast sensitivity was equal in the two eyes and fell in the upper third of the Vistech norms: binocular contrast sensitivity was in the same range.

\section{Psychometric functions}

The psychometric functions, relating percent correct responses to the spatial frequency of high-contrast. square-wave gratings, are shown for anisometropic amblyopes (Fig 1E) and strabismic amblyopes (Fig 1D) separately. The gratings were viewed through the red-green filters that were later used in the imaging experiments. The responses to horizontal and vertical gratings and the responses to gratings seen through red and green goggles were averaged.

All four anisometropic amblyopes showed subnormal psychometric functions and response latency curves (not shown) in their amblyopic eyes. The densely amblyopic subject MaM showed an interocular grating acuity difference of about two octaves; in the anisometropic amblyope EM and the strabismic and anisometropic amblyope MM, the difference in grating acuity between the two eyes was greater than one octave. Even the mildly amblyopic subject RK showed a consistent impairment in acuity and latency in his amblyopic eye. These impairments are in agreement with the Snellen visus and the contrast sensitivity functions of the subjects.

The psychometric functions of the strabismic amblyopes dissociated in some subjects from the Snellen visus and the contrast sensitivity functions. In the densely amblyopic subjects KG and RS, acuity and response latency were definitely subnormal. By contrast, in subjects KSM and PZ acuity losses and latency increases were much less pronounced than expected from the orthoptic data.

For the normal subjects the psychometric functions and the response latency curves were identical in the two eyes (not shown). The monocular grating acuily, defined as the spatial frequency yielding $75 \%$ correct responses, ranged between 36 cpd for subject NT and 20 cpd for subject EB. The nonamblyopic eyes of the anisometropic or strabismic amblyopes showed psychometric 

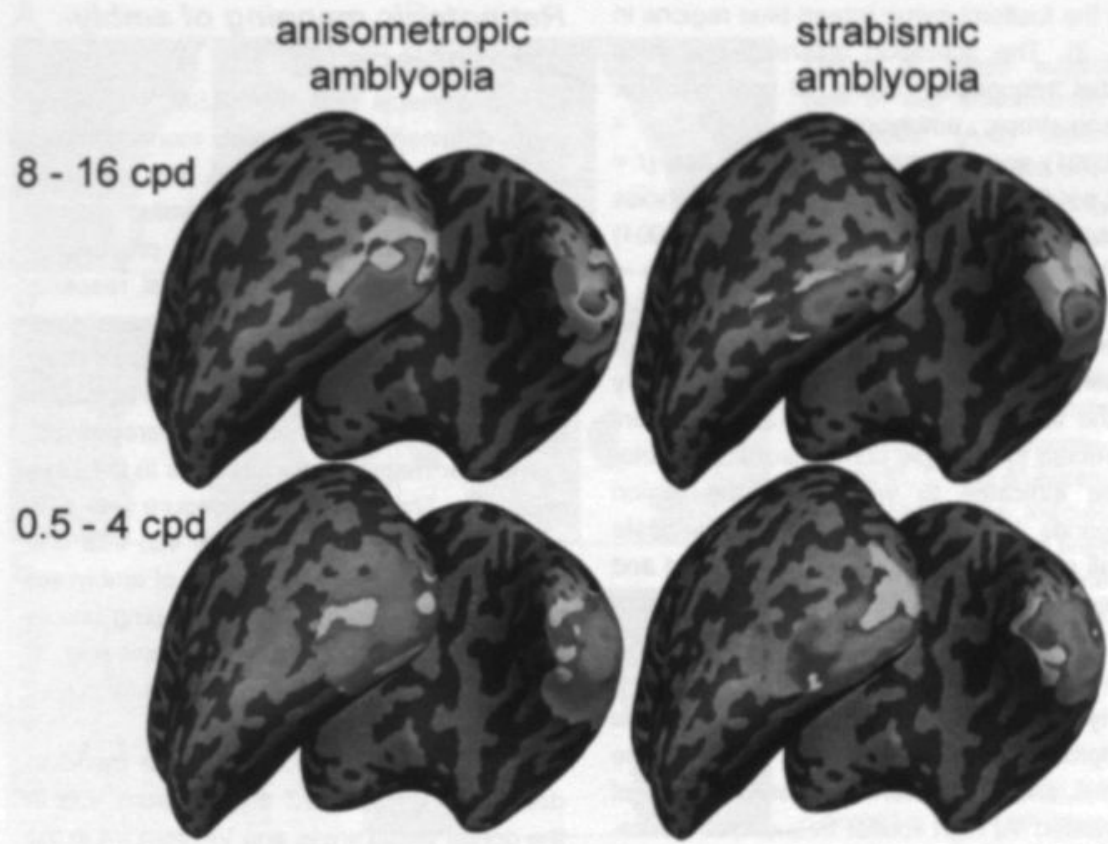

\section{+1 |IIIIIIIIIIIIIIIIII -1}

sound eye - amblyopic eye

sound eye + amblyopic eye

Figure 2. Group analysis of cortical activation of anisometropic and strabismic amblyopes. Relative contribution maps are derived from GLM-analysis and shown superimposed on inflated representation of template brain hemispheres. Maps are given for high (top row) and low (bottom row) spatial frequency and for anisometropic and (left column) and strabismic amblyopia (right) separately. Relative contribution is color coded and can vary between 1 (sound eye only) and -1 (amblyopic eye only). Color codes vary between green $(+1)$ and red $(-1)$ as indicated. Threshold for activated region is significant $(p<0.05)$ after Bonferroni correction for multiple comparison.

functions and response latency curves which were similar to those of the normal subjects ranging between $20 \mathrm{cpd}$ for MaM and $32 \mathrm{cpd}$ for RS.

\section{Group results of fMRI study}

In the groups of anisometropic and strabismic amblyopes cortical activation exhibited clear interocular differences - especially for gratings with high spatial frequencies ( $8,16 \mathrm{cpd})$. The largest interocular differences were observed in occipito-temporal regions located at the posterior end of the occipito-temporalsulcus. In these areas, the responses evoked through the amblyopic eye were consistently reduced. These regions extended laterally into the inferior temporal gyrus and ventrally 


\section{4}

into the fusiform gyrus (green-blue regions in Fig. 2). The activation differences at high spatial frequencies ( 8 and $16 \mathrm{cpd}$ ) were for anisometropic amblyopes $(t=4.7 ; p<$ $0.00001)$ and for strabismic amblyopes ( $t=$ 4.9; $p<0.000001)$. At low spatial frequencies contrast t-values were 7.2 ( $p<0.0000001)$ for anisometropic amblyopes and 5.6 (p < 0.0000001 ) for strabismic amblyopes. In Fig. 2. relative contribution maps are shown for regions that are significantly activated in any of the tested conditions ( $p<0.05$; Bonferroni corrected for multiple comparison). The color scale indicates to which eye the region responds better. Blue and yellow indicate small changes $(t<3 ; p>0.01)$, and red and green pronounced differences $(t>3 ; p<$ 0.01 ).

Early visual areas in the medial region of the occipital lobe, located around the calcarine sulcus, showed a distinctly different pattern of activation. At high spatial frequencies activation was stronger for the amblyopic than the normal eye, this difference was more pronounced for anisometropic (red color around sulcus calcarinus in Fig. 2) than for the strabismic subjects (yellow and red region in Fig. 2 top row). At low spatial frequencies activation in this region was similar for the two eyes (blue and yellow colors). In strabismic amblyopes stronger responses to the amblyopic eye was also observed in anterior parts of the sulcus calcarinus, suggesting that the peripheral sections of early visual areas might respond more strongly to the amblyopic eye than to the normal eye (see below for individual data).

In the control subjects left and right eye stimulation revealed stronger activation in the respective contralateral occipital cortex $(p<0.001)$. This laterality effect is counterbalanced in the amblyopic groups by averaging across hemispheres.

\section{Retinotopic mapping of ambly- opic subjects}

In four subjects (EM, RK, KSM, PZ) maps were obtained not only with monocular but also with binocular stimulation. The two mapping procedures gave very similar results. However, binocular mapping procedures were optimized in some technical respects (number of repetitions, TR, voxel-resolution) that improved the resolution of maps. Thus. in the four subjects we relied on the binocular maps, and in the others we averaged the monocular maps across sessions to increase reliability. Through this procedure we succeeded to map areas V1, V2, V3, V3a and VP, V4/N8 in all 16 hemispheres of amblyopic subjects. The results of this mapping procedure are shown for two anisometropic (Fig. 3) and two strabismic subjects (Fig. 4).

The representation of the vertical meridian delineates V1 from V2 and V3 from V3a in the dorsal visual areas and VP from V4 in the ventral visual areas. In Figs. 3 and 4 the representation of the lower vertical meridian appears in red and for the upper visual field in green. The horizontal meridian separates V2d from V3 and V2v from VP, respectively. Here the colors yellow and blue refer to lower (yellow) and upper (blue) visual field representations, respectively (for color codes see the small inlays of Fig $3 \mathrm{CD}$ and Fig $4 \mathrm{CD}$ ). The estimated borders are indicated by hand drawn white lines (continuous lines for horizontal meridian, dotted lines for lower vertical meridian, dashed lines for upper vertical meridian).

Areas V3a and V8 are clearly characterized by a complete hemifield representation. How ever, the anterior boundaries to V3a and V8 are difficult to map. One reason is that we did not cover the whole region in all measurements with our slab thickness of $3.3 \mathrm{~cm}$. another reason is that many repetitions are needed to reveal retinotopic maps beyond 

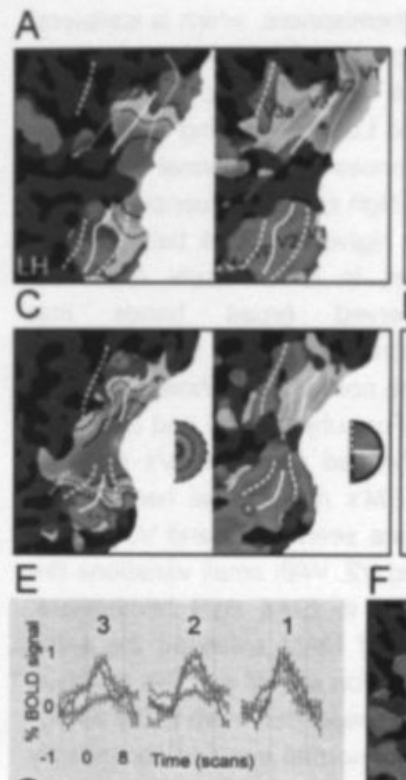

G
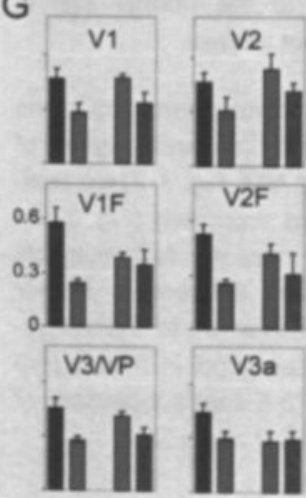
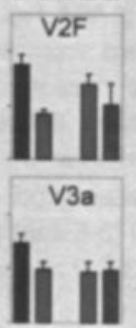

$\mathrm{F}$
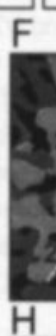

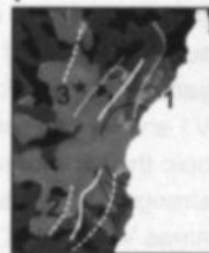

D
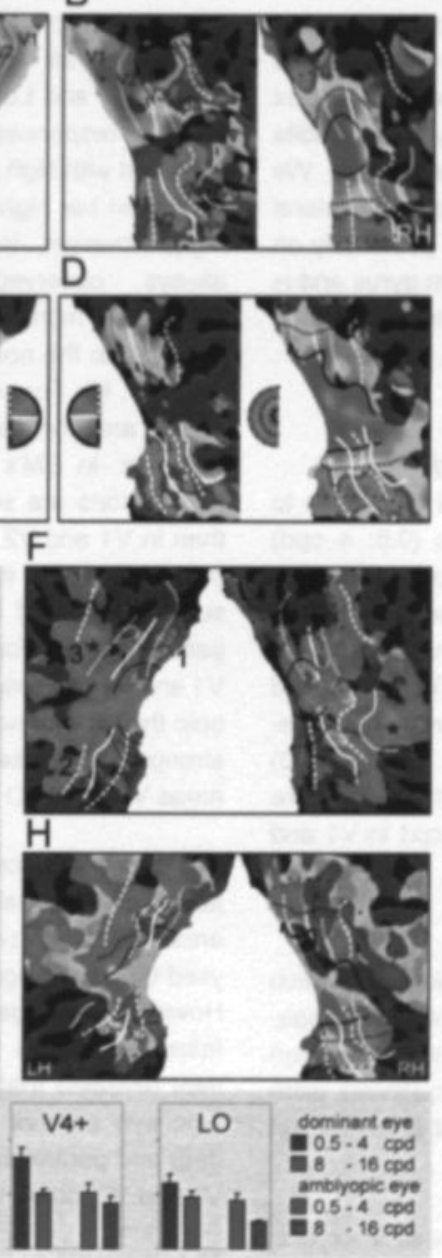

dominart tore

20.5-4 cpd a $8.16 \mathrm{cpd}$ amblyopic ere D. 0.5 .4 cpd

Figure 3. Single subject data of two anisometropic amblyopes. (A-D) retinotopic mapping of two subjects. (A) Eccentricity mapping (left) and polar angle mapping (middle) of subject MM superimposed on a flattened representation of his left occipital cortex. Dark lines indicate eccentricity lines at approximately $3^{\circ}, 5^{\circ}$ and $8^{\circ}$ eccentricity. White lines indicate area boarders (dashed lines - vertical meridian; solid line horizontal meridian). V1 is cut along its horizontal meridian (not shown) which is corresponding mostly to the calcarine sulcus. Color codes for polar mapping are indicated in (C) for left hemisphere and in (D) for the right hemisphere. The upper visual field is represented in colors from green to blue corresponding to gradients from the vertical to the horizontal meridian representation. the lower visual field is represented by colors red to yellow corresponding to gradients from vertical to horizontal meridian representation. Eccentricity color gradients vary between red

(fovea) to green (157) periphery. (B) Retinotopic mapping of the right hemisphere of subject MM. Polar-mapping middle and eccentricity mapping is given in the right penal. (C-D) Retinotopic mapping of subject EM respectively. (F, H) Contrast maps indicate regions that respond more strongly to dominant eye stimulation (green-blue) or more strongly to amblyopic eye stimulation (pink-red). using high spatial frequency gratings. (E) Examples of ROI-time courses taken from (F). (G) Averaged mean response of V1, V2, V1F (fovea), V2F, V3 and VP, V3a, V4+ and LO.

the level of V3a (Tootell et al., 1997; Tootell et al., 1998; Tootell and Hadjikhani, 2001). We suspect that the borders of V3a to presumed $V 7$ and other functional regions in the supe- rior-occipital sulcus as well as the border to V8 in the fusiform gyrus were not mapped reliably. We could ascertain, however, that the foveal representation of V8 lies ventrally 
to the foveal representation of V1-V 3 in most subjects. In cases were we could not delineate V4 and V8 we used V4+ to label the whole complex. The lateral occipital cortex was not mapped retinotopically as it exhibits only a crude retinotopic organisation. We refer to it as the region around the lateral occipital sulcus, that is located posteriorly on the lateral aspect of the fusiform gyrus and is not belonging to any of the regions exhibiting retinotopic maps (Malach et al., 1995b).

\section{Anisometropic amblyopes}

For the normal eye the cortical responses to low spatial frequency gratings $(0.5 ; 4 \mathrm{cpd})$ were always stronger than responses to high spatial frequencies (8; 16 cpd; Fig. $3 \mathrm{G}$ ). while responses to $0.5 \mathrm{cpd}$ and $4 \mathrm{cpd}$ were largely the same (not shown). This is in good agreement with the expected peak spatial frequencies reported in humans (Singh 2000) and monkeys (Foster et al., 1985) that are believed to be between 1 - $4 \mathrm{cpd}$ in V1 and slightly lower in areas V2, V3, VP (Singh et al. 2000).

Paradoxically, for the amblyopic eyes, which exhibited differential impairment for high spatial frequencies, the differences between responses to high and low frequencies were less pronounced in most visual areas except LO.

In order to assess the differences in cortical activation most closely related to the amblyopic deficit we first compared the responses to the two eyes at high spatial frequencies (8: $16 \mathrm{cpd}$ ) on flattened hemispheres for two representative subjects (Fig. 3). Regions activated more strongly from the normal than the amblyopic eye cover large parts of the occipital cortex. In subject MM differences were maximal in $\mathrm{V} 3 \mathrm{a}$. $\mathrm{V} 4+$ and $\mathrm{LO}$ of both hemispheres (Fig. $3 \mathrm{~F}$, blue region; $\mathrm{F}(1,507)>5$; $p<0.05$ ) and for subject EM in $\mathrm{V} 3 \mathrm{a}, \mathrm{V} 4+$ and
LO of the left hemisphere, which is ipsilateral to the amblyopic eye (Fig. $3 \mathrm{H} \mathrm{p}<0.05$ ). Averaged over all anisometropic subjects the areas $\mathrm{V} 4+$ and LO are showing consistently stronger responses to the normal eye when activated with high spatial frequencies (Fig. 3. G - green bar higher than pink bar). Surprisingly, however, in lower visual areas we always observed broad bands that responded more strongly to the amblyopic eye than to the normal eye (shown in pink in Fig. 3. F, H). For subjects MM and EM these bands are confined mainly to $\mathrm{V}_{1}$ and $\mathrm{V} 2$. However, in EM's right dorsal hemisphere, pink regions are seen in V3 and V3a rather than in V1 and V2. With small variations like those observed in EM's right hemisphere, subjects RK and MAM exhibited the same pattern of activation as MM and EM: bands in V1 and V2 that responded more to the amblyopic than to the normal eye and consistently stronger responses to the normal eye in areas $\mathrm{V} 4+$ and LO (not shown).

Because we noticed some differences in the activation of foveal and parafoveal regions of areas V1 and V2 (e.g. MM, Fig. 3. F) we analysed those subregions separately (Fig. 3. G). However, averages over all four subjects failed to confirm these differences. Rather they revealed that responses to the amblyopic eye are not attenuated in foveal (1-3 deg) and parafoveal (3-5 deg) subsections of V1 and V2, nor in V3, VP and V3a.

At low spatial frequencies all regions except parafoveal V1 and V2 showed a clear attenuation of responses evoked from the amblyopic eye (red and blue bars in Fig. 3. G).

\section{Strabismic amblyopes}

In all visual areas responses were stronger when evoked with low than with high frequency gratings. In areas $\mathrm{Vp}$ and $\mathrm{V} 4+$ responses evoked through the amblyopic eye were much weaker than those evoked 
A

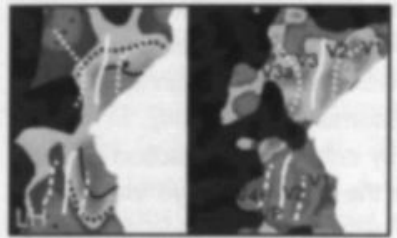

C

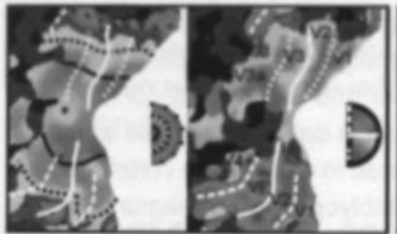

E

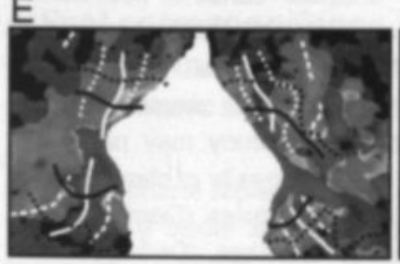

G
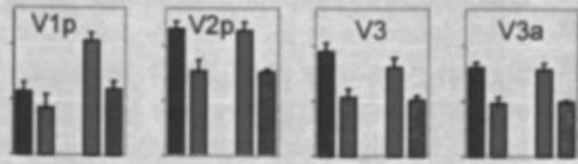

dominant eve P.5.4 cpd - $8-16 \mathrm{cpd}$ amblyopic eye 2.5.4 cpd

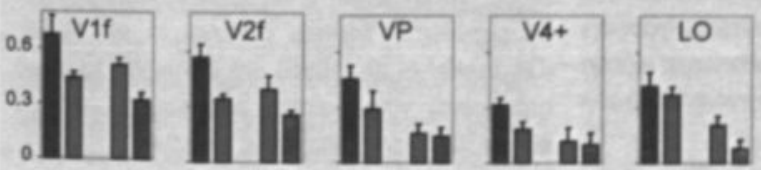

Figure 4. Strabismic amblyopia. Retinotopic mapping and high spatial frequency response shown for two strabismic amblyopes (KG. KSM) on their reconstructed and flatened cortcal sheets (subject KG: A. B, E and subject KSM: C. D. E). Lef hemisphere mapping (A, C) right hemispheres (B, D). Excentricity gradients (outer panels) are coded from red (foveal) to green (periphery). Polarangle (medial paneis) from red (lower vertical meridian) to green (upper vertical meridian). (E, F) Monocular cortical response to high spatial frequency gradients. Regions with higher cortical response to stimulation of the dominant eye are shown in green and blue. Regions with higher cortical response for amblyopic eye stimula. tion are shown in pink and red. (G) Averaged respons for four strabismic amply. opes in V1p (peripheral). V1f (N1-fovea), V2p, V2t, V3, VP, V3a, V4+, LO. through the normal eye and because of the low amplitude of these responses, differences between low and high frequency gratings could not be determined.

In extrastriate regions the activation patterns obtained with high frequency gratings resembled in many aspects those of the anisometropic subjects: Responses to stimulation of the amblyopic eye were attenuated in areas $V_{3}, V_{3} a, V_{p}, V_{4} / V_{8}$ and $L O$.
In the lower visual areas activation patterns evoked with high frequency gratings were more variable than in anisometropic amblyopes. In V1 responses were strong for all conditions but interocular differences were inhomogeneous. Some subregions showed stronger activation after stimulation of the amblyopic eye and, unlike in the anisometropic amblyopes, other subregions responded more vigorously to the normal 
eye. In three subjects there was an overall tendency for stronger responses to the amblyopic eye in regions of early visual areas representing the peripheral visual field. In one subject (KSM, Fig 3), the amblyopic eye evoked stronger responses in the fovea of v1.

\section{Discussion}

\section{Summay'}

A consistent finding was that both in anisometropic and strabismic subjects responses evoked through the amblyopic eye were attenuated in extrastriate visual areas including areas LO and V4+N8. This attenuation was more pronounced for high than for low frequency gratings and is therefore likely to be related to the amblyopic deficit which shows the same dependence on spatial frequency. In contrast, lower tier areas V1 and V2 showed no or only small and mostly foveal attenuation of responses evoked from the amblyopic eye. Unexpectedly, especially in strabismic subjects, most parts of V1 and V2 and in particular the peripheral regions responded more strongly to the amblyopic eye. Overall activation patterns in lower tier areas were more variable and patchy in strabismic than in anisometropic amblyopes while in higher tier areas the patterns of activation were similar in anisometropic and strabismic amblyopes.

\section{Activation deficits in higher visual areas}

Only few studies have investigated neuronal correlates of amblyopia in extrastriate cortical regions. In striate cortex of monkeys changes in neuronal response properties were relatively small compared to the massive behavioral deficits (for review see Kiorpes and McKee, 1999). In cats that had developed strabismic amblyopia the only noticeable def- icits in area 17 and 18 was reduced synchronisation of responses evoked from the amblyopic eye, especially when responses were evoked with gratings of high spatial frequency (Roelfsema et al., 1994). In higher visual areas, by contrast, the fraction of cells responding to the amblyopic eye was markedly reduced (PMLS and PLLS: Sireteanu and Best, 1992, area 21a: Schroder et al., 2002). In imaging studies of human subjects affected by amblyopia, activation differences between striate and extrastriate cortex were not investigated in depth. Response attenuation was reported in extrastriate cortex ipsilateral to the amblyopic eye (Imamura et al.. 1997), in the "occipital" cortex of both hemispheres (Kabasakal et al., 1995) and exclusively in 'V1" (Demer et al., 1988). The discrepancy between these results and the results of the present study may partly be accounted for by the diversity of stimuli. They consisted of motion pictures (Demer et al., 1997), stroboscopic flashes (Demer et al., 1988; Kabasakal et al., 1995), reversing checkerboard patterns (Kabasakal et al., 1995; Imamura et al., 1997) and sinusoidal gratings (Goodyear et al., 2000). Stimuli such as dramatic motion pictures are likely to preferentially activate extrastriate regions (Demer et al., 1988) while unstructured stimuli such as strabismic flashes (Demer et al., 1988; Kabasakal et al., 1995) are expected to drive only lower visual areas and seem not well suited to reveal amblyopic deficits.

Our behavioral tests revealed impaired processing only for high spatial frequency gratings and this perceptual deficit correlated best with the spatial frequency sensitive attenuation of responses in area LO. In the other extrastriate areas this attenuation was less pronounced, as e. $\mathrm{g}$. in V4 of anisometropic amblyopes or it was not dependent on spatial frequency at all as in VP and V4+ of strabismic amblyopes. Thus, the best correlation between perceptual impairment and 
response attenuation was the spatial frequency dependent response attenuation in area LO. Good correlation between perception of visual objects and LO activity has also been observed in normal subjects (Malach et al., 1995a; Grill-Spector et al., 2000; Amedi et al., 2001: Levy et al., 2001). A study of GrillSpector (2001) has shown that activity in LO is enhanced after recognition performance has been improved by training. Attenuation of LO activity in amblyopic subjects might therefore be seen as an effect of reduced training for the non-dominant eye.

\section{Effects of ethiology, anomalous retinal correspondence and fixa- tion behavior on striate response in strabismic amblyopia}

In the peripheral representation of the visual field in early visual areas V1 and V2 of the strabismic subjects the amblyopic eye evoked stronger responses than the normal eye (see group results in Fig. 2 . single subject data in Fig. 4. G. for V1p, V2p). This finding might be related to the non-uniform distribution of visual acuity and interocular suppression across the visual field that has been observed in human subjects (Sireteanu and Fronius, 1981; Sireteanu et al., 1981) and in monkeys affected by strabismic amblyopia (Horton et al., 1999; Thiele et al., 1997). It has been suggested that strabismic amblyopia results from chronic interocular suppression that occurs in regions of the visual field where fusion of the signals from the two eyes is not possible (Sireteanu and Fronius, 1981; Sireteanu, 1982). With small squint angles fusion is prevented only in the central but not in the peripheral parts of the visual field because the larger receptive fields in the periphery permit integration of binocular signals and the development of anomalous correspondence.
This could account for reduced suppression of the amblyopic eye in the periphery of the visual field representation but it does not explain why the amblyopic eye caused stronger activation than the normal eye. One possibility would be attentional modulation, assuming that subjects have to invest more effort when analyzing patterns presented to the amblyopic eye.

The enhanced activation by the amblyopic eye of peripheral field representation was most pronounced in subjects KG and PZ. These had larger squint angles than the other subjects $\left(10^{\circ}-12^{\circ}\right)$. In addition subject KG had eccentric fixation $\left(7^{\circ}-8^{\circ}\right.$ nasal) in the left amblyopic eye and both subjects had anomalous retinal correspondence. This could have enhanced peripheral activation because neurons of which the receptive fields would normally be located outside the stimulated visual field can now be activated because their fields have shifted to more medial positions. As a result a slightly larger sphere of cortex should be activated and this additional activation should manifest itself mainly in the peripheral parts of the visual field. For subjects with small squint angle (KSM and RS) peripheral effects should be small, but due to the cortical magnification factor, foveal effects could be apparent, i.e. for the amblyopic eye foveal representation is slightly larger and for the dominant eye it is more focused.

However this explanation cannot account for the unexpected finding in anisometropic subjects who showed stronger responses to the amblyopic than to the normal eye in areas V1 and V2, especially when presented with high spatial frequency gratings. As proposed above one possibility is that amblyopic subjects need to make special efforts to process the signal from the deficient eye and this could, through attentional mechanisms. enhance the BOLD-signal. That attention can enhance BOLD signals also in lower areas 
including $\mathrm{V} 1$ has been documented by Somers et al. (1999) and Brefczynski and DeYoe. (1999). However, this hypothetical increase of attentional effort did not enhance responses in higher tier visual areas which are particularly susceptible for attentional effects. One possibility is that the amblyopia related response reduction in higher areas is so pronounced that it cannot be compensated by enhanced attention. This interpretation agrees with the indications for transmission failure from lower to higher visual areas in neurophysiological experiments (Schroder et al. 2002) and the present study. However conflicts remain. A good correlation has recently been found between the amplitude of the BOLD signal and the synchronisation of neuronal responses oscillating in the gamma- frequency range (Logothetis et al. 2001). In amblyopia, however, synchronisation of responses evoked from the amblyopic eye is reduced in early visual areas (Roelfsema et al. 1994). Tthis can explain impaired perceptual grouping and reduced transmission of signals from lower to higher visual areas it predicts reduced rather than enhanced BOLD signals also in lower areas. One solution could be that attentional effects can compensate for reduced synchronisation. The experiments on response synchronisation in amblyoic cats were performed in anesthesia that precluded attentional modulation.

\section{Conclusion}

In conclusion, the most consistent result of the present study is the marked reduction of responses to stimulation of the amblyopic eye in higher visual areas of the ventral processing stream both in anisometropic and in strabismic amblyopes. This suggests transmission failure from lower to higher visual areas which is in agreement with recent data from animal experiments (Schroder et al. 2002). One reason for this reduced activation of higher areas could be reduced response synchronisation in lower areas (Roelfsema et al. 1994). Reduced synchrony lowers the saliency of neural responses because it impairs spatial summation in target cells (Usrey and Reid 1999). Moreover it is conceivable that impaired temporal correlation of responses in early areas reduces the match between the output activity of lower areas and the receptive field properties of neurones at higher areas. The response patterns in lower areas were more variable. They are consistent with the electrophysiological evidence that responses to the amblyopic eye are only little if at all affected as far as discharge rates and tuning of individual cells are concerned (Roelfsema et al. 1994). The unexpected enhancement of responses evoked through the amblyopic eye is difficult to explain on the basis of present knowledge about the relation between neuronal activity and the BOLD signal. Moreover, this enhancement exhibited high interindividual variability that we are presently unable to relate to specific perceptual deficits. However, this heterogeneity of activation patterns is not entirely unexpected since the constellation of perceptual deficits in amblyopic subjects are also highly heterogeneous and exhibit large interindividual variability. 


\section{References}

Amedi A, Malach R, Hendler T, Peled S, Zohary E (2001) Visuo-haptic object-related activation in the ventral visual pathway. Nat Neurosci 4: 324 330.

Anderson SJ, Holliday IE, Harding GF (1999) Assessment of cortical dysfunction in human strabismic amblyopia using magnetoencephalography (MEG). Vision Res 39: 1723-1738

Brefczynski JA, DeYoe EA (1999) A physiological correlate of the 'spottight' of visual attention. Nat Neurosci 2: 370-374.

Demer JL, Grafton S. Marg E, Mazziotta JC, Nuwer M (1997) Positron-emission tomographic study of human amblyopia with use of defined visual stimuli. J AAPOS 1: 158-171

Demer JL, von Noorden GK, Volkow ND, Gould KL (1988) Imaging of cerebral blood flow and metabolism in amblyopia by positron emission tomography. Am J Ophthaimol 105: 337-347

Duke-Elder S: System of Ophtaimology. Vol. V: Ocular Motility and Strabismus. London, Kimpton, 1973

Foster KH, Gaska JP, Nagler M, Pollen DA (1985) Spatial and temporal frequency selectivity of neurones in visual cortical areas V1 and V2 of the macaque monkey. J Physiol 365: 331-363

Fries $\mathrm{P}$, Reynolds JH, Rorie AE, Desimone R (2001) Modulation of oscillatory neuronal synchronization by selective visual attention. Science 291: $1560-1563$

Fries P, Roelfsema PR, Engel AK, Konig P, Singer W (1997) Synchronization of oscillatory responses in visual cortex correlates with perception in interocular rivalry. Proc Natl Acad Sci U S A 94: 12699-12704.

Goebel R, Khorram-Sefat D, Muckli L, Hacker H. Singer W (1998a) The constructive nature of vision: direct evidence from functional magnetic resonance imaging studies of apparent motion and motion imagery. Eur $\mathrm{J}$ Neurosci 10: 15631573
Goebel R, Linden DEJ, Lanfermann $H$, Zanella FE, Singer W (1998b) Functional imaging of mirror and inverse reading reveals separate coactivated networks for oculomotion and spatial transformations. Neuroreport 9: 713-719

Goebel R, Muckli L, Zanella FE, Singer W. Stoerig P (2001) Sustained extrastriate cortical activation without visual awareness revealed by fMRI studies of hemianopic patients. Vision Res 41: 1459. 1474

Goodyear BG. Nicolle DA, Humphrey GK, Menon RS (2000) BOLD fMRI response of early visual areas to perceived contrast in human amblyopia. J Neurophysiol 84: 1907-1913

Grill-Spector K, Kushnir T, Hendler T, Malach R (2000) The dynamics of object-selective activation correlate with recognition performance in humans Nat Neurosci 3: 837-843

Hess RF, Campbell FW, Zimmern R (1980) Differences in the neural basis of human amblyopias: the effect of mean luminance. Vision Res 20: 295305

Hess RF, Dakin SC, Kapoor N (2000a) The foveal 'crowding' effect physics or physiology? Vision Res 40: 365-370.

Hess RF, Dakin SC, Kapoor N. Tewfik M (2000b) Contour interaction in fovea and periphery. J Opt Soc Am A Opt Image Sci Vis 17: 1516-1524.

Hess RF. Field DJ (1994) is the spatial deficit in strabismic amblyopia due to loss of cells or an uncalibrated disarray of cells? Vision Res 34 3397-3406

Hess RF. Howell ER (1978) The luminancedependent nature of the visual abnormality in strabismic amblyopia. Vision Res 18: 931-936

Horton JC. Hocking DR (1996) Pattern of ocular dominance columns in human striate cortex in strabismic amblyopia. Vis Neurosci 13: 787-795

Horton JC, Hocking DR, Adams DL (1999) Metabolic mapping of suppression scotomas in striate cortex of macaques with experimental strabismus $\mathrm{J}$ Neurosci 19.7111 .7129

Horton JC. Stryker MP (1993) Amblyopia induced by anisometropia without shrinkage of ocular 
dominance columns in human striate cortex. Proc Natl Acad Sci U S A 90: 5494-5498

Hubel DH, Wiesel TN (1965) Binocular interaction in striate cortex of kittens reared with artificial squint. J Neurophysiol 28: 1041-1059.

Imamura K, Richter H, Fischer H, Lennerstrand G Franzen O, Rydberg A, Andersson J, Schneider $H$, Onoe $H$, Watanabe $Y$, Langstrom B (1997) Reduced activity in the extrastriate visual cortex of individuals with strabismic amblyopia. Neurosci Lett 225: 173-176.

Kabasakal L, Devranoglu K, Arslan O, Erdil TY, Sonmezoglu K, Uslu I, Tolun H, Isitman AT, Ozker K. Onsel C (1995) Brain SPECT evaluation of the visual cortex in amblyopia. J Nucl Med 36: 1170 1174

Kiorpes L, Kiper DC, O'Keefe LP, Cavanaugh JR, Movshon JA (1998) Neuronal correlates of ambly. opia in the visual cortex of macaque monkeys with experimental strabismus and anisometropia J Neurosci 18: 6411-6424

Kiorpes L, McKee SP (1999) Neural mechanisms undertying amblyopia. Curr Opin Neurobiol 9 480-486.

Kriegeskorte N, Goebel R (2001) An efficient algorithm for topologically correct segmentation of the cortical sheet in anatomical MR volumes Neurolmage 14:329-346.

Lagreze WD, Sireteanu R (1991) Two-dimensional spatial distortions in human strabismic amblyopia. Vision Res 31: 1271-1288.

Leopold DA, Logothetis NK (1996) Activity changes in earty visual cortex reflect monkeys' percepts during binocular rivaliry. Nature $379: 549$ 553

Levy I. Hasson U. Avidan G. Hendler T. Malach R (2001) Center-periphery organization of human object areas. Nat Neurosci 4:533-539

Levi DM, Manny RE (1982) The pathophysiology of amblyopia: electrophysiological studies. Ann N Y Acad Sci 388. 243-263.

Linden DEJ, Kallenbach U., Heinecke A. Singer W. Goebel R (1999) The myth of upright vision. A psychphysical and functional imaging study of adaptation to inverting spectacles. Perception 28: 469-481.

Logothetis NK, Schall JD (1989) Neuronal correlates of subjective visual perception. Science 245 : 761-763.

Malach R, Reppas JB, Benson RR, Kwong KK, Jiang $H$, Kennedy WA, Ledden PJ, Brady TJ, Rosen BR, Tootell RB (1995a) Object-related activity revealed by functional magnetic resonance imaging in human occipital cortex. Proc Natl Acad Sci U S A 92: 8135-8139

Malach R, Reppas JB, Benson RR, Kwong KK, Jiang $H$, Kennedy WA, Ledden PJ, Brady TJ, Rosen BR, Tootell RB (1995b) Object-related activity revealed by functional magnetic resonance imaging in human occipital cortex. Proc Natl Acad Sci U S A 92: 8135-8139

Movshon JA, Eggers HM, Gizzi MS, Hendrickson AE, Kiorpes L. Boothe RG (1987) Effects of early unilateral blur on the macaque's visual system. III Physiological observations. J Neurosci 7: 1340 1351

Rauschecker JP, Singer W (1979) Changes in the circuitry of the kitten visual cortex are gated by postsynaptic activity. Nature 280: 58-60.

Roelfsema PR, Konig P, Engel AK, Sireteanu R Singer W (1994) Reduced synchronization in the visual cortex of cats with strabismic amblyopia Eur J Neurosci 6: 1645-1655.

Schroder JH, Fries P, Roelfsema PR. Singer W. Engel AK (2002) Ocular dominance in extrastriate cortex of strabismic amblyopic cats. Vision Res 42: 29-39

Sereno MI, Dale AM, Reppas JB, Kwong KK, Belliveau JW. Brady TJ, Rosen BR, Tootell RB (1995) Borders of multiple visual areas in humans revealed by functional magnetic resonance imag. ing. Science 268: 889-893.

Sharma V. Levi DM. Coletta NJ (1999) Sparsesampling of gratings in the visual cortex of strabismic amblyopes. Vision Res 39: 3526-3536

Sharma V, Levi DM, Klein SA (2000) Undercounting features and missing features: evidence for a high-level deficit in strabismic amblyopia. Nat Neurosci 3: 496-501 
Sheinberg DL, Logothetis NK (1997) The role of temporal cortical areas in perceptual organization. Proc Natl Acad Sci U S A 94: 3408-3413.

Singer W, von Grunau M, Rauschecker J (1980) Functional amblyopia in kittens with unilateral exotropia. I. Electrophysiological assessment Exp Brain Res 40: 294-304

Singh KD, Smith AT, Greenlee MW (2000) Spatiotemporal frequency and direction sensitivities of human visual areas measured using MRI. Neuroimage $12: 550-564$

Levi DM, Manny RE (1982) The pathophysiology of amblyopia: electrophysiological studies. Ann N Y Acad Sci 388: 243-263.

Sireteanu R (1982) Human amblyopia: consequence of chronic interocular suppression. Hum Neurobiol 1: 31-33

Sireteanu R, Best J (1992) Squint-induced modification of visual receotive fields in the lateral suprasylvian cortex of the cat Binocular interaction, vertical effect and anomalous correspondence. Eur J Neurosci 4: 235-242.

Sireteanu R, Fronius M (1981) Naso-temporal asymmetries in human amblyopia consequence of long-term interocular suppression. Vision Res 21: $1055-1063$

Sireteanu R, Fronius M, Singer W (1981) Binocular interaction in the peripheral visual field of humans with strabismic and anisometropic ambly. opia. Vision Res 21: 1065-1074

Somers DC. Dale AM, Seiffert AE, Tootell RB (1999) Functional MRI reveals spatially specific attentional modulation in human primary visual cortex. Proc Natl Acad Sci U S A 96: 1663-1668

Thiele A, Bremmer F, lig UJ. Hoffmann KP (1997) Visual responses of neurons from areas $\mathrm{V}_{1}$ and MT in a monkey with late onset strabismus: a case study. Vision Res 37: 853-863.

Tootell RB, Hadjikhani N (2001) Where is 'dorsal $\mathrm{v4}^{\prime}$ in human visual cortex? retinotopic, topographic and functional evidence. Cereb Cortex 11. 298-311

Tootell RB, Mendola JD, Hadjikhani NK, Ledden PJ, Liu AK, Reppas JB, Sereno MI, Dale AM (1997) Functional analysis of V3A and related areas in human visual cortex. J Neurosci 17 7060-7078

Tootell RBH, Hadjikhani NK, Mendola JD, Marrett S. Dale AM (1998) From retinotopy to recognition: fMRI in human visual cortex. Trends Cognit Sci 2 : 174-183

Usrey WM, Reid RC (1999) Synchronous activity in the visual system. Annu Rev Physiol 61: 435456 .

Wesel TN, Hubel DH (1965) Extent of recovery from the effects of visual deprivation in kittens. J Neurophysiol 28: 1060-1072

\section{Acknowledgments}

We thank David E. J. Linden for valuable comments on the manuscript and Ariane Fiesser for help on data processing. 


\section{Chapter 3}

\section{Sustained extrastriate cortical activation without visual awareness revealed by fMRI studies of hemianopic patients}

This chapter corresponds to:

Rainer Goebel ${ }^{1}$, Lars Muckli ${ }^{2}$, Friedhelm E. Zanella ${ }^{3}$. Wolf Singer ${ }^{2}$, Petra Stoerig ${ }^{4}$ (2001) Sustained extrastriate cortical activation without visual awareness revealed by fMRI studies of hemianopic patients. Vision Research 41, 10-11, 1459-1474.

'Department of Cognitive Neuroscience, Faculty of Psychology, Maastricht University. Maastricht, The Netherlands. ${ }^{2}$ Max Planck Institute for Brain Research, Frankfurt a. M., Germany. ${ }^{3}$ Klinikum der Johann Wolfgang Goethe-Universitat, Institut fur Neuroradiologie, Frankfurt a. M., Germany. ${ }^{4}$ Institute of Experimental Psychology II, Heinrich-Heine-University. Dusseldorf. Germany. 


\section{Abstract}

Patients with lesions in the primary visual cortex (V1) may show processing of visual stimuli presented in their field of cortical blindness even when they report being unaware of the stimuli. To elucidate the neuroanatomical basis of their residual visual functions, we used functional magnetic resonance imaging in two hemianopic patients, FS and GY. In the first experiment, a rotating spiral stimulus was used to assess the responsiveness of dorsal stream areas. Although no response was detectable within denervated or destroyed early visual cortex, motion-sensitive areas (hMT+N5) ipsilateral to the lesion showed a strong sustained hemodynamic response. In GY, this activation was at least as strong as that of his contralesional hMT+/N5 to the stimulus in the normal hemifield. In the second experiment, coloured images of natural objects were used to assess the responsiveness of ventral stream areas. Again, no activity was detectable in ipsilesional early visual areas, but extrastriate areas in the lateral occipital cortex (hMT+N5 and LO) and within the posterior fusiform gyrus (V4/N8) showed a robust sustained hemodynamic response. In both experiments, we observed that ipsilesional areas responded to stimuli presented in either hemifield, whereas the normal hemisphere responded preferentially to stimuli in the sighted hemifield. As only one subject occasionally noticed the onset of stimuluation in the impaired field, the unexpectedly strong sustained activity in ipsilesional dorsal and ventral cortical areas appears to be insufficient to generate conscious vision. 


\section{Introduction}

The term 'blindsight' describes the ability of neurological patients with postgeniculate lesions to detect, localise and discriminate visual stimuli in blind parts of the visual field. although they do not consciously perceive them (Weiskrantz, Warrington, Sanders, \& Marshall, 1974). These residual visual functions must be mediated by those parts of the visual system that retain visual responsiveness despite the lesion that destroys or denervates primary visual cortex (V1) and causes cortical blindness. Data from monkeys have shown that despite retrograde degeneration of projection cells in the dorsal lateral geniculate nucleus (dLGN) and the retina (Van Buren, 1963; Cowey, Stoerig. \& Perry, 1989), subcortical retinorecipient nuclei continue to receive visual input from the parts of the retinae that represent the cortically blind visual field (for a recent review, see Stoerig \& Cowey, 1997). Some of these nuclei project directly to extrastriate visual cortex, which consists of cytoarchitectonically distinct areas that subserve specific visual functions (Zeki, 1974; Zilles \& Clarke, 1997). Evidence indicates that these areas are organized within two main visual pathways (Mishkin, Ungerleider, \& Macko, 1983): a ventral stream or 'what' system devoted to the finegrained analysis of the visual scene, including processing of shape and colour and a dorsal stream or 'where' system, which processes spatial characteristics of the visual scene and analyses motion. Emphasizing that the latter pathway is also involved in visuomotor transformations, Milner and Goodale (1995) proposed to distinguish between 'vision for perception' (ventral pathway) and 'vision for action' (dorsal pathway). The two pathways originate in areas $V_{1}$ and V2 and extend into the temporal (the 'what' system) and parietal (the 'where' system) lobe, respectively. With brain imaging techniques, they have been convincingly demon- strated in the normal human brain (e.g. Haxby et al., 1991) and several specialized areas have been identified on the basis of their stimulus preference profiles.

An extensively studied area in the ventral stream is area V4, which in the macaque, appears to be involved in the processing of chromatic and shape information (see Cowey, 1994 for a review). It has been reported to mediate colour constancy, but nevertheless, its necessity for colour vision is debated on the grounds that ablation of this area does not produce marked deficits in colour discrimination (Heywood \& Cowey. 1987). In man, the colour complex has been located in the fusiform gyrus (Lueck et al., 1989; Hadjikhani, Liu, Dale, Cavanagh, \& Tootell, 1998), in a region that is generally compromised in patients with achromatopsia (Meadows, 1974) and is often referred to as V4 (McKeefry \& Zeki, 1997; Zeki, McKeefry, Bartels, \& Frackowiak, 1998) or V8 (Hadjikhani et al., 1998); we shall refer to it here as the 'colour complex' or V4N8. Other areas located in close proximity to area V4N8 have been characterized, including the fusiform face area (FFA, Kanwisher, Dermott, \& Chun, 1997) and several overlapping regions sensitive to different object categories (e.g. Ishai, Ungerleider, Martin, Schouten \& Haxby, 1999). Less specialized, with respect to object categories but responsive to common objects, abstract sculptures and famous faces is a more dorsal area in a region at the lateral-anterior aspect of the occipital lobe that Malach et al. (1995) called LO (lateral occipital complex).

Of the dorsal stream areas, those that are specialized for motion processing have received particular attention. In monkey cortex, the motion-selective area MT (middle temporal area) is located in the middle temporal sulcus, close to the junction of the occipital, temporal and parietal lobes (Van 
Essen, Maunsell \& Bixby, 1981). Its cells are responsive to the direction and speed of moving stimuli (Snowden, Treue \& Andersen, 1992) and their inactivation causes deficiencies in motion direction discrimination. Area MT is easily identified in histological sections because of its high myelination and it is surrounded by several other specialized areas ('satellites') that process higher-order aspects of stimulus motion. The human visual cortex contains an area that is likely to correspond to monkey area MT and is located in the occipito-temporo-parietal pit. Bilateral lesions, that include this area, cause a severe impairment in detecting the movement of objects (cortical akinetopsia, see Zeki, 1991). Imaging studies have shown that metabolism and blood flow in that region increases more in response to moving than to stationary stimuli, indicating preferential involvement of this area in the processing of motion information (e.g. Watson et al., 1993; Tootell et al., 1995; Goebel, Khorram-Sefat, Muckli, Hacker, \& Singer, 1998a). As functional imaging studies have not yet convincingly differentiated between the human homologue of area MT and its satellites, this motion-selective region is generally referred to as the "human motion complex' ( $\mathrm{hMT}+$ ) or as V5.

Although area MT receives its major input directly or indirectly from the primary visual cortex (V1), destruction or temporary inactvation of V1 in monkeys does not eliminate its visual responsiveness (Rodman, Gross \& Albright, 1989). Although the responses were markedly reduced, a large part of the neuronal population remained visually responsive and even retained directional tuning. Accordingly, one might expect residual visual responsiveness in the motion complex of human patients who have suffered lesions to the primary visual cortex, and indeed, functional imaging studies in patients with lesions of the optic radiation or primary visual cortex have confirmed its continued responsive- ness to moving or flickering stimuli (Barbur, Watson, Frackowiak, \& Zeki, 1993; Stoerig. Goebel, Muckli, Hacker, \& Singer, 1997; Zeki \& ffytche, 1998). In contrast to dorsal stream areas, neurons in the monkey's ventral stream become unresponsive to stimuli in the blind field after V1 lesions (Girard, Salin \& Bullier, 1991; Bullier, Girard \& Salin, 1993). Thus, one would expect little, if any, activity in areas of the ventral stream in patients with lesions to the primary visual cortex. However, in a recent imaging study of two hemianopic patients, we have found that presentation of images of natural objects in the cortically blind visual field can lead to strong sustained activity in the ventral pathway without any detectable activity in primary visual cortex (Goebel et al., 1998b; Goebel, Muckli, Zanella, Singer, \& Stoerig, submitted).

In the present study, we compare the responsiveness of dorsal and ventral (Goebel et al., submitted) stream areas after blind field stimulation in the two hemianopic patients. GY and FS, as measured by echoplanar functional magnetic resonance imaging. To activate dorsal stream areas, a rotating spiral stimulus was presented in the cortically blind visual field. We used a rotating spiral instead of translational motion (Sahraie et al., 1997: Zeki \& ffytche, 1998) because it restricts motion to a limited region of space for prolonged stimulation epochs (e.g. $30 \mathrm{~s})$. This allowed us to separate the hemodynamic response to stimulus onset, which GY can be aware of (see Barbur, Ruddock, \& Waterfield. 1980) from the sustained response to the rotatory motion. For activation of ventral stream areas, coloured images of natural objects (firit and vegetables) were used (Goebel et al., submitted). Both stimulus types were also presented in the sighted visual field of the patients to allow for a direct within-subject comparison of the hemodynamic response to stimulation in the cortically blind and in the intact visual fieid. As a further 


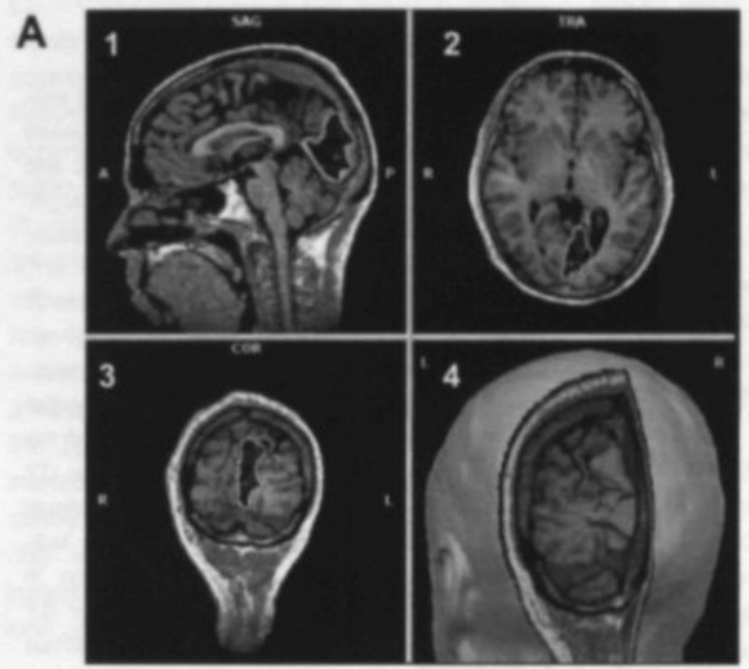

B
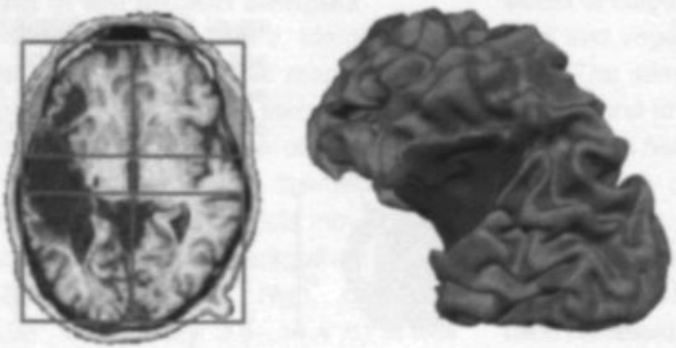

Fig. 1. Lesions of patients GY (A) and FS (B). (A) Slices of an anatomical $\mathrm{T} 1$-weighted MRI scan showing the V1 lesion of GY. The small cross in $(1-3)$ indicates the 30 position of the selected slices. the yellow curve delineate the extend of the lesion within the respective slices. (1) Sagittal slice through the lesion. (2) Axial slice through the lesion. (3) Coronal slice through the lesion. (4) Surface rendering of GYs reconstructed head (yellow) and of the boundary of the V1 lesion (red). The rendered head was partially opened to reveal the size and position of the lesion. (B) Rendering of the reconstructed cortical surface at the boundary between grey and white matter of the affected left hemisphere of patient FS. An image containing a single slice running through the lesion is also shown. control, the same experiments were conducted with two healthy subjects. In addition, retinotopic mapping experiments were performed to describe the precise spatial layout of the functional responsiveness of early visual areas in the normal and lesioned hemisphere.

\section{Methods}

\section{Patients}

FS and GY, who participated in this study, have long-standing post-geniculate lesions of the left hemisphere (see Fig. 1). FS, born in 1937. suffered a severe craniocerebral trauma when he was 42 years of age. GY, born in 1956, was involved in a traffic accident when he was 8 years, and his left primary visual cortex was almost completely destroyed by a vascular incident. The visual field defects that resulted from the lesions are shown in Fig. 2. The wedge-shaped region in FS's right hemifield (black) is absolutely blind, while GY's hemianopia is relative and allows conscious detection of salient visual events. Over many years, GY and FS have participated in studies of their residual visual functions (e.g. for GY: Barbur et al., 1980; Blythe, Bromley, Kennard, \& Ruddock, 1986; Brent, Kennard, \& Ruddock, 1994; Weiskrantz, Barbur, \& Sahraie, 1995; Morland, Ogilvie, Rud- 
A

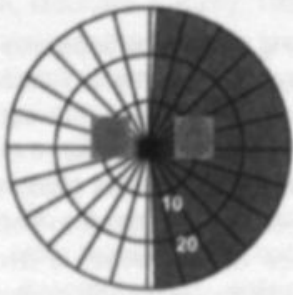

B

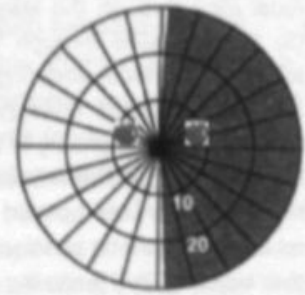

GY

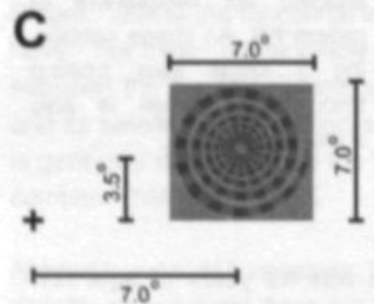

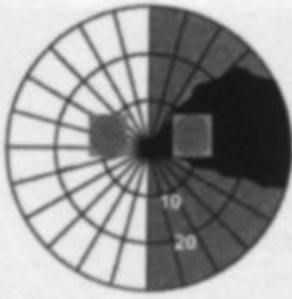

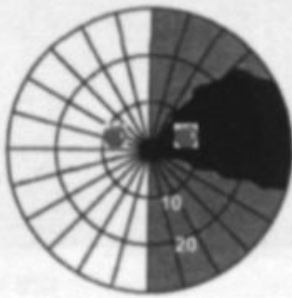

FS

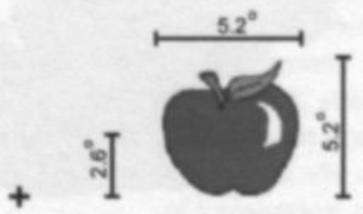

Fig. 2. Plots of visual field sensitivity (A, B) which are based on a combination of static and dynamic perimetry (target: 116', 320 cyc deg ${ }^{-1} \mathrm{~m}^{-2}$, background: $10 \mathrm{cyc}^{-1} \mathrm{deg}^{-1} \mathrm{~m}^{-2}$. white: normal field, black: absolutely blind field, grey. relatively blind region, see text). (A) Visual field sensitivity plot with indicated position of the moving spiral stimulus for GY (left) and FS (right). (B) Visual field sensitivity plot with indicated stimulation position in the objects experiment for $\mathrm{GY}$ (left) and FS (right). Stimuli were shown in separate blocks in either the left or the right visual field. (C) Stimulus size and location in the spiral experiment (left) and in the objects experiment (right).

\section{$70^{\circ}$}

dock, \& Wright, 1996; Finlay, Jones, Morland, Ogilvie, \& Ruddock, 1997; Marcel, 1998; Guo, Benson, \& Blakemore, 1998; Benson, Guo, \& Blakemore, 1998; Kentridge, Heywood, \& Weiskrantz, 1999 and for FS: Poppel, 1985, 1986; Stoerig. 1993; Stoerig \& Cowey, 1997; Stoerig. Kleinschmidt, \& Frahm, 1998). The lesions were visualized by MR-imaging (Fig. 1) using a high-resolution T1-weighted 3D FLASH sequence (voxel size $1 \times 1 \times 1 \mathrm{~mm})$ recorded with a $1.5 \mathrm{~T}$ scanner (Siemens Magnetom Vision). FS's extensive lesion primarily affects the temporal lobe and includes the optic radiation in the vicinily of the LGN. In GY, the medial occipital lobe of the left hemisphere is destroyed, including most of V1, as well as surrounding extrastriate visual cortex and the underlying white matter, but sparing the occipital pole.

\section{Tasks}

\section{Retinotopic mapping}

The responsiveness and delineation of early visual areas V1, V2, V3, VP, V3A. V4V and $\mathrm{V} 4 \mathrm{~d}$ was investigated with retinotopic mapping scans (cf. Sereno, Dale, Reppas. Kwong, Belliveau, Brady, Rosen, \& Tootell, 1995), sampling 12 contiguous slices approximately in parallel to the calcarine fissure. 
Eccentricity and polar angle mapping experiments were performed in the same recording session (for details see Linden, Kallenbach, Heinecke, Singer, \& Goebel, 1999). For eccentricity mapping, a ring-shaped configuration of black and white contrast-reversing $(8 \mathrm{~Hz})$ checkers was presented centered around the fixation point. The ring started with a radius of $1^{\circ}$ visual angle and expanded to a radius of $12^{\circ}$ within $96 \mathrm{~s}$. For polar angle mapping, the same pattern was configured as a wedge subtending $22.5^{\circ}$ in polar angle with the tip at the fixation cross. The wedge started at the left horizontal meridian and slowly rotated clockwise for a full cycle of $360^{\circ}$ within $96 \mathrm{~s}$. Each mapping experiment consisted of four repetitions of a full expansion and a full rotation.

\section{Mapping of the motion complex}

Motion-selective areas were identified by comparing the hemodynamic response during presentation of a moving stimulus with the response during presentation of a stationary control stimulus. We used a flowfield stimulus, consisting of $\mathbf{4 0 0}$ white dots moving radially outward on a black background (visual field: $28^{\circ}$ wide by $20^{\circ}$ high, dot size: $0.06 \times 0.06^{\circ}$, dot velocity: $3.6-14.4^{\circ} / \mathrm{s}$ ). It was contrasted with a stationary dot display to produce a clear response of motion-sensitive areas (Tootell et al., 1995).

\section{Motion experiment (Experiment 1)}

While the flowfield stimulated both hemifields simultaneously, the main stimulus was presented $6.8^{\circ}$ off-axis in one hemifield at a time. It consisted of a blue-and-red (run 1) or black-and-white (run 2) checkered spiral (Fig. 2A,C), $7^{*}$ in diameter, rotating counter-clockwise around its centre $\left(180^{\circ} / \mathrm{s}\right)$. Each stimulafion block lasted for $30 \mathrm{~s}$ and was repeated four times within each run. In an additional condition, a stationary spiral was shown four times in four blocks of $30 \mathrm{~s}$. All stimulation blocks were separated by fixation blocks of equal length. The stimulus was presented on a grey background to prevent light scatter from the stimulus into the sighted hemifield. A schematic drawing of the stimulus configuration is shown in Fig. 2(A,C).

\section{Object experiment (Experiment 2)}

Coloured images of natural objects (fruit and vegetables) subtending $5.2 \times 5.2^{*}$ were presented in the left (normal) or right (impaired) upper visual field, $7^{*}$ offaxis (Fig. 2B). Each stimulation block lasted for $30 \mathrm{~s}$ and was repeated four times within each recording session. Stimulation blocks were separated by fixation blocks of equal length. Within a stimulation block, an image was shown for 1 $s$ and then replaced by the next image without an interstimulus interval. Images consisted of coloured drawings of natural objects (fruit and vegetables, Corel Draw clip art gallery). The stimuli were presented on a white background to minimize stray light falling into the sighted hemifield. A schematic drawing of the stimulus configuration is shown in Fig. 2(B,C).

In order to assess whether the subjects were aware of the stimuli presented in the cortically blind hemifield, we asked them to respond by button press when they detected anything in the right visual hemifield.

\section{MRI acquisition}

Functional magnetic resonance imaging was performed at 1.5 T (Siemens Magnetom Vision) using the standard head coil and a gradient echo EPI sequence. The Siemens Magnetom gradient overdrive allowed functional scans with high spatial and temporal resolution ( $T E=69 \mathrm{~ms}, \mathrm{TR}=3000 \mathrm{~ms}, \mathrm{FA}=90^{\circ}$. $F O V=200 \times 200 \mathrm{~mm}^{2}$, matrix size: $128 \times 128$, voxel size: $1.6 \times 1.6 \times 3-5 \mathrm{~mm}^{3}$ ). A T1weighted $3 D$ MP RAGE scan lasting 8 min was performed in the same session (voxel size $1 \times 1 \times 1 \mathrm{~mm}^{3}$ ). An additional T1-weighted 
3D data set tuned to optimize the contrast between gray and white matter was recorded in a separate recording session lasting 24 min (FLASH sequence). The intrasession MP RAGE 3D data set was automatically aligned with the extrasession T1 FLASH data set. Visual stimuli were delivered under computer control (Digital DECpc Celebris XL 590) to a high-luminance LCD projector (EIKI LC6000 ). The image was backprojected onto a frosted screen positioned at the foot of the scanner. Visual stimuli were generated using the Microsoft DirectX graphics library and a Matrox Mystique graphics board.

\section{Data analysis}

FMRI data analysis was performed using BrainVoyager 3.x/2000 (Brain Innovation. Maastricht, The Netherlands, unw. BrainVoyager.com) and included removal of low-frequency drifts, 3D motion detection and correction, determination of Talairach coordinates, multiple regression analysis, cortex reconstruction, inflation and flattening. For the retinotopic mapping experiments, crosscorrelation analysis was applied (for details see Linden et al., 1999). The eccentricity and polar angle represented by a given cortical site was determined by finding the lag value maximizing the cross-correlation. The obtained lag values at each voxel, corresponding to the eccentricity or polar angle of optimal stimulation, were encoded in pseudocolours on slices as well as on surface patches (triangles) of the reconstructed cortical sheet (see below). Pixels were included into the statistical map if the obtained crosscorrelation value $r$ was $>0.4 \quad(P<0.0001$, uncorrected). In order to detect weak activity within and surrounding the lesioned or denervated regions, retinotopic mapping experiments were also analysed with a lowered correlation threshold of $r>0.2$. Based on the polar angle, mapping the boundaries of retinotopic cortical areas V1, V2, V3, VP, V3A and
$\mathrm{V} 4 \mathrm{v}$ were estimated on the flattened cortical surface maps (see below) of each patient's non-lesioned hemisphere and of both hemispheres in each control subject. The motion complex mapping experiment was analysed with a simple correlation analysis contrasting the flowfield stimulus with the stationary control stimulus.

The two main experiments were analysed with a multiple regression model consisting of two predictors, one for the presentation of the stimulus (moving spiral/ images of natural object) in the left and one for the presentation of the stimulus in the right visual field. The overall model fit was assessed using an F statistic. The obtained P-values were corrected for multiple comparisons using a cortex-based Bonferroni adjustment, i.e. the number of voxels included for correction was limited to grey matter voxels (Goebel \& Singer, 1999). The relative contribution of each of the two predictors $R C=(b 1-b 2)$ / (b1+b2) was visualised with a red-yellowgreen pseudocolour scale. Statistical maps were superimposed on the original functional scans and incorporated into the high-resolution 3D MRI data sets through interpolation to the same resolution (voxel size: $1 \times 1 \times 1 \mathrm{~mm}$ ). Since the 2D functional and 3D structural measurements were acquired in the same recording session, coregistration of the respective data sets could be performed on the basis of the Siemens slice position parameters of the $\mathrm{T} 2^{*}$-weighted measurement (number of slices, slice thickness, distance factor, Tra- Cor angle, FOV, shift mean, offcenter read, offcenter phase, in-plane resolution) and the T1-weighted 3D MP RAGE measurement (number of sagittal partitions, shift mean, offcenter read, offcenter phase, resolution). In order to compare activated brain regions across sessions, anatomical and functional $3 D$ data sets were transformed into Talairach space (Talairach \& Tournaux, 1988). Results were visualised by superim- 
posing 3D statistical maps on reconstructions of each patient's cortical sheet. Areas were identified based on their anatomical position and Talairach coordinates.

The recorded high-resolution T1-weighted 3D recordings were used for surface reconstruction of both cortical hemispheres of the control subjects and patients (for details see Linden et al., 1999; Kriegeskorte \& Goebel, 2001). The white/grey matter border was segmented with a region-growing method preceded by inhomogeneity correction of signal intensity across space. The border of the resulting segmented subvolume was tessellated to produce a polygon-mesh surface reconstruction of each cortical hemisphere. The tessellation of the white/grey matter boundary of a single hemisphere typically consists of $\sim 250000$ triangles. An iterative 3D morphing algorithm (Goebel et al., 1998a) was used to move the vertices outward along the surface normals into the grey matter. Through visual inspection, this process was halted when the surface reached the middle of the gray matter corresponding approximately to layer 4 of the cortex. The resulting surface was used as the reference mesh for projecting functional data on folded, inflated or flattened representations. A morphed surface was always linked to its folded reference mesh, so that functional data could be shown at the correct location of an inflated or flattened representation. This link was also used to minimize geometric distortions during inflation and flattening (Goebel, 2000) by inclusion of a morphing force that keeps the distances between vertices of each triangle of the morphed surface as close as possible to the respective values of the folded reference mesh.

\section{Results}

Results from a control subject of the retinotopic mapping. motion complex mapping and objects experiment are shown in Fig. 3 .
Results of the two patients are shown in Figs. 4-6.

\section{Retinotopic mapping}

The topography of the early visual areas is shown in Fig. 3a for a normal observer. In the patients, the contralesional V1 appeared normal, but no visual responsiveness was seen in its lesioned (GY) or denervated (FS) counterpart $(r>0.4, P<0.00001)$. Only a small region at the occipital pole was activated in GY (Fig. 5a, see also Baseler, Morland, \& Wandell, 1999). This is in agreement with GY's visual field perimetry that shows a $3^{\circ}$ macular sparing in the lower quadrant of the hemianopic right visual field (Barbur et al., 1980).

\section{Motion complex mapping}

The comparison of the flowfield stimulus with the stationary dot stimulus revealed several motion-selective areas in both hemispheres including the motion complex, area V $3 \mathrm{~A}$ and an area at the border between occipital and parietal lobes (Fig. 3b). In GY, the motion complex in the ipsilesional hemisphere is located more posteriorly and more ventrally than in the contralesional hemisphere. The position of the motion complex in both patients (Table 1) agrees with previous reports in normal observers (Tootell et al., 1995; Goebel et al., 1998a).

\section{Motion experiment}

In the control subjects, the foci of activation in the lateral occipital cortex produced by passive viewing of the moving spiral were functionally identified as hMT+N5 because they overlapped with the regions activated by the flowfield stimulus (not shown). When the moving spiral stimulus was presented in the cortically blind (right) visual field of the patients, the ipsilesional (left) motion complex 


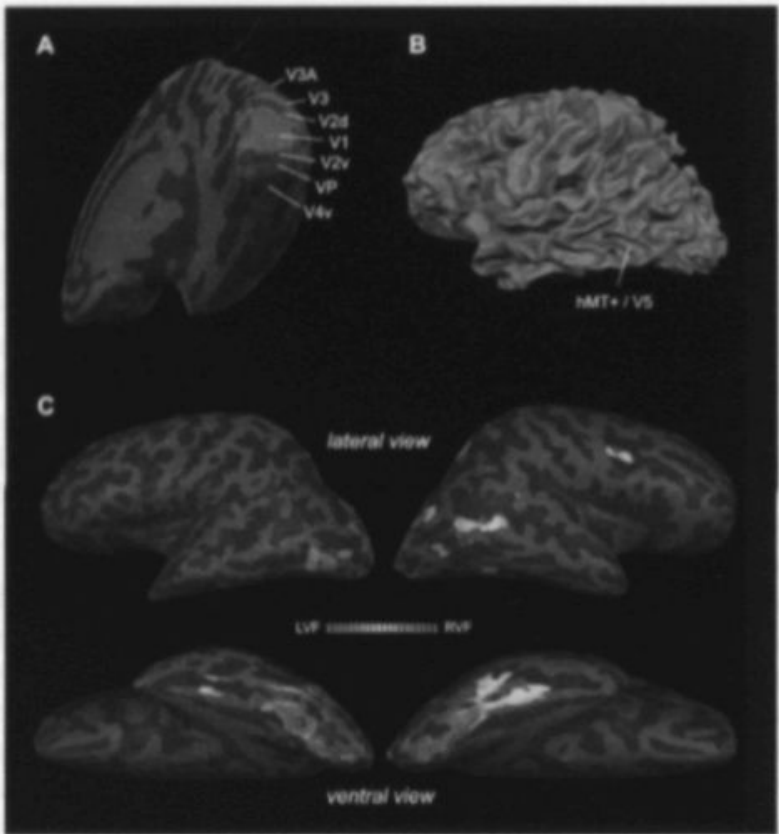

Fig. 3. Results of a control subject of the retinotopic mapping experiment (A). motion complex mapping experiment (B) and objects experiment (C). (A) Boundaries of retinotopic cortical areas were determined through field sign map computations (cf. Sereno et al. 1995) based on eccentricity and polar angle mapping. (B) Lateral view of the reconstructed cortical sheet. The statistical map comparing the flowfieid condition with the stationary dots condition is projected on the surface revealing motion-selective areas including V3A and hMT+N5. (C) Lateral and ventral view of "inflated" cortical hemispheres. Activated regions responding solely to ts presented in the left visual field are coloured green; regions responding solely to objects presented in ight visual field are coloured red. Areas responding with equal strength to stimuli in either hemifield are $m$ in yellow (for graded values, see colour scale).

was strongly activated (Fig. 4) despite the fact that there was no detectable activation within and around the lesioned or denervated region. A weaker but significant response was also observed in the contralesional motion complex. When the spiral stimulus was presented in the left (sighted) hemifield, both the contralesional motion complex, as well as the ipsilesional motion complex, responded strongly (see Fig. 4). Thus, the motion complex at the ipsilesional side responded equally strong (FS) or stronger (GY) to the moving spiral presented in the cortically blind visual field, whereas the response of the contralesional motion complex was stronger to the spiral in the sighted (left) visual field in both patients.

In $<20 \%$ of the presentations of the rotating spiral in the cortically blind hemifield, GY pressed the button for a short duration following the onset of the spiral, indicating that he was aware of a stimulus change. An analysis of the data restricted to the first $15 \mathrm{~s}$ of the 30 s stimulation epochs produced almost identical results as the analysis restricted to the last 15 s. FS never indicated awareness when stimuli were presented in his blind visual field.

In GY, the comparison of the hemodynamic response to the presentation of the moving spiral in the cortically blind visual fieid and the sighted visual field revealed that the extent of the functionally defined motion complex in the lesioned hemisphere was greater than in the intact hemisphere (Table 2). Interestingly. this difference was more pronounced in the 


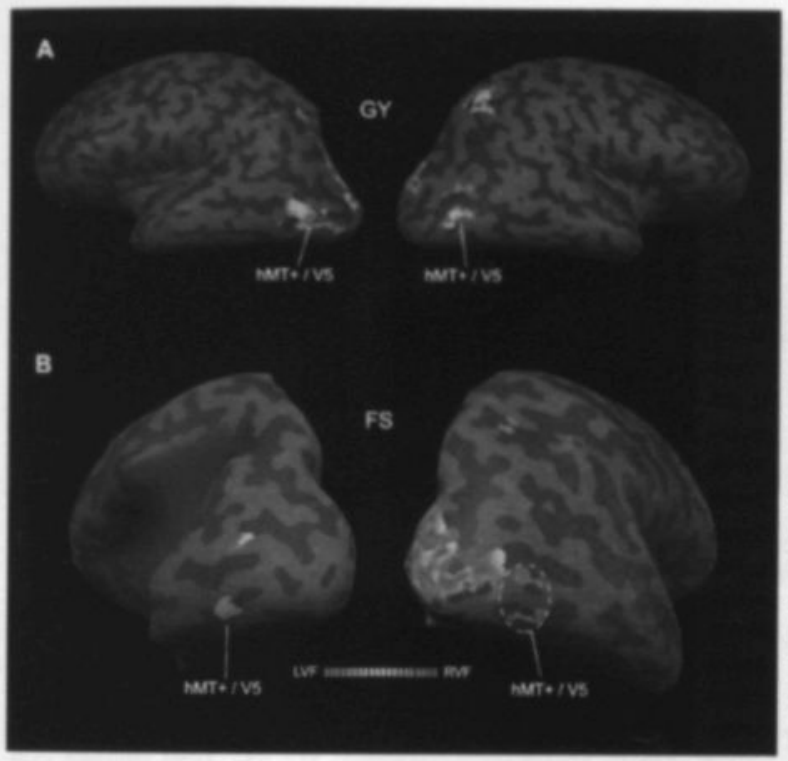

Fig. 4. Multiple regression results of the black-and-white spiral experiment of patient GY (A) and patient FS (B), shown on inflated representation of the cortical sheet. Activated regions responding solely to the spiral presented in the left visual field are coloured green; regions responding solely to the spiral presented in the right visual field are coloured red. Areas responding with equal strength to the spiral in either hemifield are shown in yellow (for graded values, see colour scale). (A) Activated regions in the right hemisphere are dominated by green colors whereas regions in the lesioned left hemisphere are dominated by yellow colors. The extent of the functionally defined motion complex is greater in the ipsilesional hemisphere than in the intact hemisphere. Time courses of left and right hemispheric motion complex (hMT+N5) for left hemifield stimulation (green curves) and right hemifield stimulation (red curves). (B) Activated regions in the right hemisphere are dominated by green colors whereas regions in the lesioned left hemisphere are dominated by yellow colors.

black-white spiral experiment than in the redblue spiral experiment. The red-blue spiral, although not isoluminant, possessed much less luminance contrast than the black-white spiral. We quantified the observation of an extended ipsilesional motion complex in GY using the number of activated voxels as well as the maximal correlation values in the obtained statistical maps (Table 2). During presentation of the moving spiral in the cortically blind visual field, the maximum singlevoxel correlation value was $r_{\max }=0.62$ (blackwhite spiral) and $r_{\max }=0.61$ (red-blue spiral) and located in the ipsilesional motion complex. With a fixed single-voxel correlation value of $>0.4$ ( $P<0.00001$, uncorrected), the cluster size (CS=number of activated voxels) was $C_{r>0.4}=93$ (black-white spiral) and $\mathrm{CS}_{p 0.4}=61$ (red-blue spiral). During presen- tation of the moving spiral in the sighted visual hemifield, the maximum single-voxel correlation value was $r_{\max }=0.55$ (black-white spiral) and $r_{\max }=0.60$ (red-blue spiral) and located in the contralesional motion complex. With a value of $C S_{n 04}=40$, the cluster size was also larger for the red-blue than the black-white spiral $\left(\mathrm{CS}_{p 04}=26\right)$, indicating that in the normal hemisphere, chromatic contrast is more effective than in the lesioned one.

\section{Object experiment}

In the control subjects, activated regions in early visual areas responded solely to objects presented in the contralateral visual field (Fig. $3 c$ ). With increasing distance from V1, visual areas responded also to stimuli in the ipsilat- 


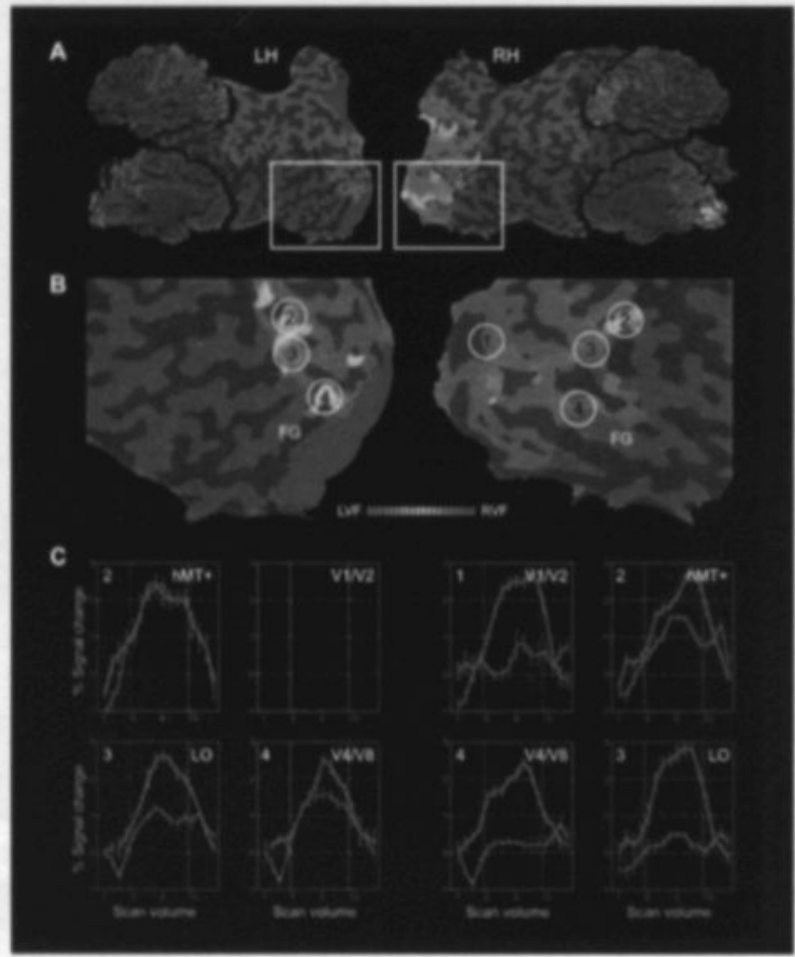

Fig. 5. Multiple regression results of object experiment of patient GY. Dark red regions in $(A)$ and $(B)$ correspond to the boundary of the cortex and the lesion; LH=left hemisphere, $\mathrm{RH}=$ right hemisphere. (A) Folded (small insets) and flattened representation of the reconstructed cortical sheet with superimposed results of eccentricity mapping: foveal to peripheral visual field representations are coded in colour from red to yellow to blue to green. (B) Results of the object experiment superimposed on a subpart of the flattened cortex representation of each hemisphere as indicated with white rectangles in (A). Activated regions responding solely to objects presented in the left visual field are coloured green, regions responding solely to objects presented in the right visual field are coloured red. Areas responding with equal strength to stimuli in either hemifield are shown in yellow (for graded values, see colour scale); FG=fusiform gyrus. (C) Time courses of right hemispheric area V1N2 and left and right hemispheric areas hMT+N5, presumed LO and presumed V4N8 for left hemifield stimulation (green curves) and right hemifield stimulation (red curves); location of plotted areas are indicated by numbers $1-4$ in (B).

eral visual field as indicated in Fig. $3 \mathrm{c}$ by the change from red to yellow color (left hemisphere) and green-to-yellow color (right hemisphere). Regions responding equally well to stimuli in both visual fields (yellow) were more extended in the right hemisphere. The human motion complex, which is known to respond not only to motion but also to flickering stimuli (Tootell et al., 1995) also responded strongly to the presentation of the images. The motion complex in either hemisphere responded to stimuli in both visual fields albeit with a stronger response to the respective contralateral hemifield.
The normal hemisphere in the patients responded to stimuli in the normal hemifield with a similar activation pattern as that seen in the control subjects, and includes early visual areas, the lateral occipital cortical region and a region in the fusiform gyrus (Fig. $5 \mathrm{~b}$ and Fig. 6b), right hemisphere). Activity in the lesioned hemisphere caused by stimuli in the normal field (Fig. 5b-c and Fig. 6b-c, green curves) was less extended than in the controls, but in GY, the region in the fusiform gyrus responded as well as it did to stimuli in the normal hemifield. Much to our surprise, 


\begin{tabular}{lllllllllllll}
\hline \multicolumn{10}{c}{ GY LH } & \multicolumn{1}{c}{ GY RH } & \multicolumn{1}{c}{ FS LH } & \multicolumn{1}{c}{ FS RH } \\
\hline hMT+/V5 & -36 & -67 & 1 & 47 & -63 & 0 & -48 & -76 & 0 & 47 & -64 & -3 \\
LO & -39 & -73 & -15 & 45 & -65 & -20 & -38 & -67 & -8 & 43 & -65 & -16 \\
V4/V8 & -25 & -65 & -12 & 22 & -62 & -21 & -35 & -62 & -18 & 28 & -53 & -17 \\
\hline
\end{tabular}

Table 1. Talairach coordinates $(x$, $y, z)$ of regions hMT+N5, LO, and V4N8 in the lef (LH) and right (RH) hemisphere of patients $G Y$ and FS.

ventral areas in the lesioned hemishere responded quite strongly to images presented in the impaired (right) hemifield (Fig. $5 \mathrm{c}$ and Fig. 6c, red curves).. In GY, both a region in the fusiform gyrus and the lateral occipital region were activated. These foci presumably correspond to V4/V8 and area LO, respectively, as their Talairach coordinates (Table 1) closely match those reported in the literature (Malach et al., 1995; Hadjikhani et al., 1998). Time courses show that the ipsilesional response strength in area V4/ V8 is nearly the same for stimuli in the blind and sighted field, but the response of area LO is weaker for stimuli in the blind field (Fig. 5c). In FS, the lateral occipital region in the lesioned hemisphere responded roughly half as strong to images in the impaired hemifield compared to the response after sighted field stimulation (Fig. 6c), but there was only little activation in V4N8. In both subjects, some activation was also seen in hMT+N5. In GY, bilateral hMT+N5 activity was observed after stimulation in either hemifield (Fig. 5b-c), but in FS, only stimulation of the normal hemifield elicited bilateral hMT+N5; no significant activity was observed after stimulation in the blind visual field (Fig. 6b-c). Throughout, the normal hemisphere showed very little activafion in response to stimuli in the impaired field, indicating that the coactivation of corresponding areas commonly seen in higher extrastriate cortical areas is compromised. In contrast to control subjects, no activity was observed anterior to V4N8 in the left. lesioned hemisphere in either patient.

In $<10 \%$ of the presentations of the objects in the cortically blind hemifield, GY pressed the

\begin{tabular}{|c|c|c|c|c|}
\hline & \multicolumn{2}{|c|}{ Black-white spiral } & \multicolumn{2}{|c|}{ Red-blue spiral } \\
\hline & $I_{\max }$ & $\mathrm{CS}_{\mathrm{ma4}}$ & $I_{\text {mex }}$ & $\mathrm{CS}_{\mathrm{n}=04}$ \\
\hline RH & -39 & -73 & 45 & -65 \\
\hline LH & 0.02 & 93 & 22 & -62 \\
\hline
\end{tabular}

Table 2. Two measures characterizing the response strenght in hMT+N5 in the left, lesioned hemisphere after blind field stimulation and in the right hemisphere after stimula. tion in the sighted hemifield maximum observed correlation value $\left(r_{\max }\right)$ and cluster size (number of connected, activated pixels) at a specified correlation value of $r=0.4\left(\mathrm{CS}_{m=0.4}\right)$

button for a short duration following the onset of the image sequence, indicating that he was aware of a stimulus change. An eventrelated analysis of 'aware' versus 'unaware' epochs produced almost identical results. As in the motion experiment, FS never indicated awareness when stimuli were presented in his blind visual field.

\section{Discussion}

\section{Summary}

We studied activation patterns in dorsal and ventral cortical areas in response to a rotating spiral and to images of natural objects presented in the intact and cortically blind fields of two hemianopic patients. We found surprisingly strong responses to blind-field stimulation in ipsilesional extrastriate areas including the motion complex, but also in ventral areas, which probably correspond to area LO and V4/N8. There was no detectable activity in the early visual cortical areas of the 


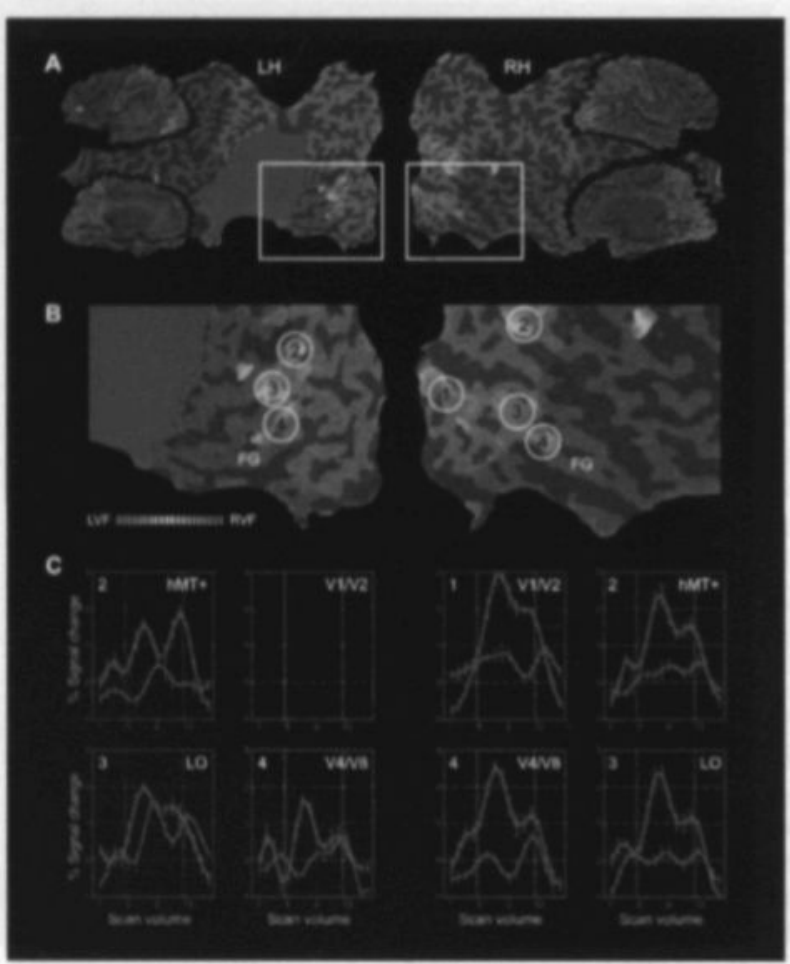

Fig. 6. Multiple regression results of object experiment of patient FS. Dark red regions in (A) and (B) correspond to the boundary of the cortex and the lesion; LH=left hemisphere, RH=right hemisphere. (A) Folded (small insets) and flattened representation of the reconstructed cortical sheet with superimposed results of the main experiment. (B) Results of the object experiment superimposed on a subpart of the flattened cortex representation of each hemisphere as indicated with white rectangles in (A). Activated regions responding solely to objects presented in the left visual field are coloured green, regions responding solely to objects presented in the right visual field are coloured red (for graded values, see colour scale): FG=fusiform gyrus. (C) Time course plots of areas of interest as in Fig. 5; location of plotted areas are indicated by numbers $1-4$ in (B)

lesioned hemisphere and with the exception of GY's occasional button press at stimulus onset, the patients indicated no awareness of the stimuli. In addition, the data revealed an unexpected asymmetry: the areas of the intact hemisphere responded well to stimulation of the contralateral, sighted hemifield but very little to stimulation of the impaired hemifield. In contrast, the corresponding areas in the lesioned hemisphere responded to stimulation of either hemifield.

\section{Extrastriate cortical activation without V 1}

A strong and sustained activity in extrastriate areas is not what one would have expected on the basis of previous monkey experiments which showed that responses of neurons in MT, while still present, are markedly reduced (Rodman et al., 1989) and that neurons in ventral stream areas become unresponsive to visual stimuli presented in the affected hemifield (Girard et al., 1991; Bullier et al., 1993). It appears unlikely that the observed activation is mediated by only partially damaged V1 tissue (Fendrich, Wessinger, \& Gazzaniga, 1992; Wessinger, Fendrich, \& Gazzaniga, 1997) since several other functional imaging studies have also shown an absence of detectable activation in the lesioned cortex in GY (see Barbur et al. 1993; Sahraie et al., 1997; Zeki \& fytche. 1998: Baseler, Morland, \& Wandell, 1999: 
Keiser, Wittsack, Niedeggen, Goebel, \& Stoerig. 2001) and the denervated region in FS (Stoerig et al., 1998; Kleiser et al., 2001) after blind field stimulation. Nevertheless, of the two findings, the dorsal activation of hMT + is less surprising. It has been reported before (Barbur et al., 1993; Sahraie et al., 1997; Stoerig et al., 1997; Zeki \& ffytche, 1998) and it agrees well with psychophysical data showing that 'dorsal' functions, such as localization (Poppel, Frost \& Held, 1973; Weiskrantz et al., 1974) and motion processing (Barbur et al., 1980; Poppel, 1985; Blythe et al., 1986; Perenin, 1991; Benson et al., 1998) can be demonstrated in cortically blind fieids. In view of the physiological results from monkey studies (Rodman, Gross \& Albright, 1990), it is likely that the information reaches hMT+ via the colliculo-pulvinoextrastriate cortical pathway. What is unexpected is the extent of ipsilesional hMT+ activation in GY, which, different from FS, is actually as pronounced as that of the motion complex in the intact hemisphere when it is stimulated from the normal hemifield. The strength of activation runs counter the reduction seen in the physiological data from monkeys with ablated or cooled $\mathrm{V} 1$ and may indicate plastic changes of the system compensating for the loss of V1 which is normally the major (direct and indirect) source of hMT+ input. When compared to FS, in whom the activation was weaker on the lesioned side, one may suggest that GY's much earlier lesion allowed for better reorganization. That it is luminance rather than chromatic contrast that reveals the extent of GY's hMT+ activation could be seen as a consequence of the degenerative effects of a $\mathrm{V} 1$ lesion on the colour-opponent retino-geniculo-striate cortical system that originates in the PB-ganglion cells of the retina (Cowey et al., 1989): The magnocellular luminance- and motion-processing system feeding into the dorsal pathway is much less compromised.
In view of the data from monkeys, whose V1 had been deactivated (Bullier et al., 1993). the activation of ventral cortical areas from stimulation of the impaired field is most unexpected. In the absence of V1, the information could reach the ventral areas either via direct subcortical projections, as those from the degenerated lateral geniculate nucleus (Yukie \& Iwai, 1981; Cowey \& Stoerig. 1989). or it could arise via lateral dorso-ventral connections. Our data do not allow refutation of either hypothesis, although the latter is rendered somewhat less likely by the weakness of the object-induced activity seen in hMT+. Preliminarily, we have attributed this hMT+ activation to the image presentation frequency of $1 \mathrm{~Hz}$ which represents a slow flicker. Ventral' functions, such as chromatic (Poppel, 1986; Stoerig. 1987; Stoerig \& Cowey, 1992; Brent et al., 1994) and shape discrimination (Weiskrantz et al., 1974; Weiskrantz, 1987; Marcel, 1998; Stoerig, in preparation, 1998) have also been demonstrated in cortically blind visual fields, although they are generally more difficult to elicit than the dorsal functions, and require more testing to reveal weaker, albeit sometimes highly significant, results. Our results indicate that it may be the human colour complex V4N8 that mediates the residual processing of chromatic information, as chromatic information activates this region (Lueck et al., 1989; Hadjikhani et al., 1998) and its destruction entails achromatopsia (Meadows, 1974). This assumption fits well with findings showing good chromatic processing in GY (Brent et al., 1994 Barbur et al., 1999 Stoerig, in preparation), but poorer processing in FS (Stoerig. 1987; in preparation) whose V4/V8 was not detectably activated during blind field stimulation. The ipsilesional lateral occipital area LO that responded in both subjects could be involved in residual form pro- cessing, although the psychophysical predictions suggested by the 
response properties of LO - better response to objects than textures, comparable responses to faces, objects, and abstract sculptures (Malach et al., 1995), and reduction of activity in response to scrambled natural objects (Grill-Spector et al., 1998) - have not yet been tested.

\section{Asymmetry of extrastriate corti- cal activation}

While we found that each hemisphere was activated by its contralateral visual hemifield. activation by the ipsilateral hemifield was asymmetrical for the two hemispheres. Though this pattern was less obvious in dorsal areas, the ventral foci of the intact hemisphere responded much less to stimulation of the impaired hemifield than did those of the lesioned hemisphere to stimulation of the normal hemifield. In addition, the activation in the lesioned ventral areas is quite focal: it does not extend forward or backward as it does in the intact hemisphere. It is possible that these extrastriate areas, while able to respond to stimuli in the impaired field, are incapacitated with respect to conveying activity forward, backward, or to the other hemisphere. Such an inability to effectively transmit signals to other areas might be caused by inactivation of recurrent loops between higher and early visual areas. Recurrent loops can organize neuronal activity into stable resonant states. which have been proposed as the neural correlate of conscious vision (e.g. Tononi \& Edelman, 1998: Grossberg. 1999; Engel \& Singer, 2001). As we found sustained and pronounced cortical activity in both subjects without awareness of the presented stimuli, our data are in agreement with such 'resonance' theories, but not with simple 'activation threshold theories' (Palmer, 1999), which assume that any cortical neural activity sufficiently strong or lasting will produce conscious experience of the content it represents. 


\section{References}

Barbur, J. L., Ruddock, K. H., \& Waterfield, V. A. (1980). Human visual responses in the absence of the geniculo-calcarine projection. Brain, 103, 905-928.

Barbur, J. L., Watson, J. D., Frackowiak, R. S., \& Zeki, S. (1993). Conscious visual perception without V1. Brain, 116, 1293-1302.

Baseler, H. A., Morland, A. B., \& Wandell, B. A. (1999). Topographic organization of human visual areas in the absence of input from primary cortex. Journal of Neuroscience, 19, 2619-2627.

Benson, P. J., Guo, K., \& Blakemore, C. (1998). Direction discrimination of moving gratings and plaids and coherence in dot displays without primary visual cortex (V1). European Journal of Neuroscience, 10. 3767-3772.

Blythe, I. M., Bromley, J. M., Kennard, C., \& Ruddock, K. H. (1986). Visual discrimination of target displacement remains after damage to the striate cortex in humans. Nature, 320 . $619-621$.

Brent, P. J., Kennard, C., \& Ruddock, K. H. (1994). Residual colour vision in a human hemianope: spectral responses and colour discrimination. Proceedings of the Royal Society London. Series B : Biology Science, 256, 219-225.

Bullier, J., Girard, P., \& Salin, P.-A. (1993). The role of area 17 in the transfer of information to extrastriate visual cortex. In A. Peters, \& K. S. Rockland. Cerebral cortex, vol. 10 (pp. 301-330). New York: Plenum Press.

Cowey, A. (1994). Cortical visual areas and the neurobiology of higher visual processes. In M. J. Farah, \& G. Ratcliff, The Neuropsychology of high-level Vision (pp. 3-31). Hillsdale, $\mathrm{NJ}$ : Lawrence Eribaum.

Cowey, A., \& Stoerig. P. (1989). Projection patterns of surviving neurons in the dorsal lateral genicualte nucleus following discrete lesions of striate cortex: implications for residual vision. Experimental Brain Research, $75,631-638$

Cowey, A., Stoerig. P., \& Perry, V. H. (1989). Transneuronal retrograde degeneration of retinal ganglion cells after damage to striate cortex in macaque monkeys: selective loss of P beta cells. Journal of Neuroscience, 29. 65-80.

Engel, A. K., \& Singer, W. (2001). Temporal binding and the neural correlates of sensory awareness. Trends in Cognitive Sciences, 5. 16-25.

Fendrich, R., Wessinger, C. M., \& Gazzaniga, M. S. (1992). Residual vision in a scotoma. Implications for blindsight. Science, 258. 1489-1491.

Finlay, A. L., Jones, S. R., Morland, A. B., Ogilvie, J. A. \& Ruddock, K. H. (1997). Movement in the normal visual hemifield induces a percept in the 'blind' hemifield of a human hemianope. Proceedings of the Royal Society London. Series B, 264, 267-275

Girard, P., Salin, P. A. \& Bullier, J. (1991). Visual activity in macaque area $V_{4}$ depends on area 17 input. Neuroreport, 2, 81-84.

Goebel, R. (2000). A fast automated method for flattening cortical surfaces. Neurolmage. 11. S680 Abstract.

Goebel, R., \& Singer, W. (1999). Cortical surface-based statistical analysis of functional magnetic resonance imaging data. Neurolmage, 9, S64 Abstract.

Goebel, R., Khorram-Sefat, D., Muckli, L., Hacker, H., \& Singer, W. (1998a). The constructive nature of vision: direct evidence from functional magnetic resonance imaging studies of apparent motion and motion imagery. European Journal of Neuroscience, 10. 1563-1573.

Goebel, R., Stoerig. P., Muckli, L., Zanella, F. E., \& Singer, W. (1998b). Ipsilesional visual activation in ventral extrastriate cortex in patients with blindsight. Society for Neuroscience Abstracts, 24, 1508

Goebel, R., Muckli, L, Zanella, F.E., Singer, W. \& Stoerig. P. (submitted). Sustained acti- 
vation without visual awareness in ipsilesional ventral cortical areas of hemianopic patients.

Grill-Spector, K., Kushnir, T., Hendler, T., Edelman, S., Itzchak, Y., \& Malach, R. (1998). A sequence of object-processing stages revealed by fMRI in the human occipital lobe. Human Brain Mapping. 6, 316-328.

Grossberg. S. (1999). The link between brain learning, attention, and consciousness. Conscious Cognition, 8, 1-44.

Guo, K., Benson, P. J., \& Blakemore, C. (1998). Residual motion discrimination using colour information without primary visual cortex. NeuroReport, 9, 2103-2106.

Hadjikhani, N., Liu, A. K., Dale, A. M., Cavanagh, P., \& Tootell, R. B. H. (1998). Retinotopy and color sensitivity in human visual cortical area V8. Nature Neuroscience, 1. 235-241.

Haxby, J. V., Grady, C. L., Horwitz, B., Ungerleider, L. B., Mishkin, M., Carson, R. E., Herscovitch, P., Schapiro, M. B., \& Rapoport, S. I. (1991). Dissociation of spatial and object visual processing pathways in human extrastriate cortex. Proceedings of the Natural Academy of Science USA, 88, 1621-1625.

Heywood, C. A., \& Cowey, A. (1987). On the role of cortical area V4 in the discrimination of hue and pattern in macaque monkeys. Journal of Neuroscience, 7, 2601-2617.

Ishai, A., Ungerleider, L. G. Martin, A. Schouten, J. L., \& Haxby, J. V. (1999). Distributed representation of objects in the human ventral visual pathway. Proceedings of the Natural Academy of Science USA. 96(16), 9379-9384.

Kanwisher, N., McDermott. J., \& Chun, M. M. (1997). The fusiform face area: a module in human extrastriate cortex specialized for face perception. Journal of Neuroscience, 17. 4302-4311

Kentridge, R. W. Heywood, C. A., \& Weiskrantz. L. (1999). Effects of temporal cueing on residual visual discrimination in blindsight. Neuropsychologia, 37, 479-483.

Kleiser, R., Wittsack, J., Niedeggen, M., Goebel, R. \& Stoerig. P. (2001). Is V1 necessary for conscious vision in areas of relative cortical blindness? Neurolmage, 13(4), 654 661.

Kriegeskorte, N. \& Goebel, R. (2001). An efficient algorithm for topologically correct segmentation of the cortical sheet in anatomical MR volumes. Neurolmage, 14 (2), 329-346.

Linden, D. E. J., Kallenbach, U., Heinecke, A., Singer, W., \& Goebel, R. (1999). The myth of upright vision. A psychophysical and functional imaging study of adaptation to inverting spectacles. Perception, 28, 469-481.

Lueck, C. J., Zeki, S., Friston, K. J., Deiber. M. P., Cope, P., Cunningham, V. J., Lammertsma, A. A., Kennard, C., \& Frackowiak. R. S. (1989). The colour centre in the cerebral cortex of man. Nature, 340, 386-389.

Malach, R., Reppas, J. B., Benson, R. R. Kwong, K. K., Jiang. H., Kennedy, W. A., Ledden, P. J., Brady, T. J., Rosen, B. R., \& Tootell, R. B. (1995). Object-related activity revealed by functional magnetic resonance imaging in human occipital cortex. Proceedings of the Natural Academy of Science USA. 92. 8135-8139.

Marcel, A. J. (1998). Blindsight and shape perception: deficit of visual consciousness or of visual function? Brain, 121, 1565-1588.

McKeefry, D. J., \& Zeki, S. (1997). The position and topography of the human colour centre as revealed by functional magnetic resonance imaging. Brain, 120, 2229-2242.

Meadows, J. C. (1974). Disturbed perception of colours associated with localized cerebral lesions. Brain, 97, 615-632.

Milner, A. D.. \& Goodale, M. A. (1995). The visual brain in action. Oxford: Oxford University Press. Mishkin, M., Ungerleider, L. G. \& Macko, K. A. (1983). Object vision and spatial 
vision: two cortical pathways. Trends in Neuroscience, $6,414-417$.

Morland, A. B., Ogilvie, J. A., Ruddock, K. H., \& Wright, J. R. (1996). Orientation discrimination is impaired in the absence of the striate cortical contribution to human vision. Proceedings of the Royal Society London. Series B, 263, 633-640.

Palmer, S. E. (1999). Vision science. Cambridge, MA: MIT Press. Perenin, M. T. (1991). Discrimination of motion direction in perimetrically blind fields. Neuroreport, 2, 397-400.

Poppel, E. (1985). Bridging a neuronal gap. Perceptual completion across a cortical scotoma is dependent on stimulus motion. Naturwissenschaften, 72, 599-600.

Poppel, E. (1986). Long-range colour-generating interactions across the retina. Nature, $320,523-525$.

Poppel, E., Frost, D., \& Held, R. (1973). Residual visual function after brain wounds involving the central visual pathways in man. Nature, 243, 295-296.

Rodman, H. R., Gross, C. G. \& Albright. T. D. (1989). Afferent basis of visual response properties in area MT of the macaque. I. Effects of striate cortex removal. Journal of Neuroscience, 9, 2033-2050.

Rodman, H. R., Gross, C. G. \& Albright, T. D. (1990). Afferent basis of visual response properties in area MT of the macaque: 2 . Effects of superior colliculus removal. Journal of Neuroscience, 10, 1154-1164.

Sahraie, A., Weiskrantz, L., Barbur, J. L., Simmons, A., Williams, S. C. R., \& Brammer, M. J. (1997). Pattern of neuronal activity associated with conscious and unconscious processing of visual signals. Proceedings of the Natural Academy of Science USA, 94. 9406-9411.

Sereno, M. I., Dale, A. M., Reppas, J. B., Kwong. K. K., Belliveau. J. W. Brady, T. J. Rosen, B. R., \& Tootell, R. B. H. (1995). Borders of multiple visual areas in humans revealed by functional magnetic resonance imaging. Science, 268, 889-893.

Snowden, R. J., Treue, S., \& Andersen, R. A. (1992). The response of neurons in areas V1 and MT of the alert rhesus monkey to moving random dot patterns. Experimental Brain Research, 88, 389-400.

Stoerig. P. (1987). Chromaticity and achromaticity. Evidence for a functional differentiation in visual field defects. Brain, 110, 869886.

Stoerig. P. (1993). Spatial summation in blindsight. Vision Neuroscience, 10, 1141-1149.

Stoerig. P. (in prep.) Apples and oranges: Discrimination of images of natural objects in patients with retro-geniculate lesions.

Stoerig. P., \& Cowey, A. (1992). Wavelength discrimination in blindsight. Brain, 115, 425444. Stoerig. P., \& Cowey, A. (1997). Blindsight in man and monkey. Brain, 120, 535559.

Stoerig, P., Goebel, R., Muckli, L., Hacker, H., \& Singer, W. (1997). On the functional neuroanatomy of blindsight. Social Neuroscience Abstracts, 23, 845.

Stoerig. P., Kleinschmidt, A., \& Frahm, J. (1998). No visual responses in denervated V1: high-resolution functional magnetic resonance imaging of a blindsight patient. Neuroreport, 9, 21-25.

Talairach, J., \& Tournaux, P. (1988). Co-planar stereotaxic atlas of the human brain. New York, NY: Thieme.

Tononi, G. \& Edelman, G. M. (1998). Consciousness and complexity. Science, 282. 1846-1851.

Tootell, R. B. H., Reppas, J. B., Kwong. K. K., Malach, R., Born, R. T., Brady, T. J., Rosen, B. R., \& Belliveau, J. W. (1995). Functional analysis of human MT and related visual cortical areas using magnetic resonance imaging. Journal of Neuroscience, 15, 3215-3230.

Van Buren, J. M. (1963). Trans-synaptic retrograde degeneration in the visual system of 
primates. Journal of Neurology, Neurosurgery and Psychiatry, 34, 140-147.

Van Essen, D. C., Maunsell, J. H. R., \& Bixby, J. L. (1981). The middle temporal visual area in the macaque: myeloarchitecture, connections, functional properties and topographic organization. Journal of Cognitive Neurology. 201, 81-98.

Watson, J. D. G. Myers, R., Frackowiak, R. S. J., Hajnal, J. V., Woods, R. P., Mazziotta, J. C., Shipp, S. \& Zeki, S. (1993). Area V5 of the human brain: evidence from a combined study using positron emmission tomography and magnetic resonance imaging. Cerebral Cortex, 3, 79-94.

Wessinger, C. M., Fendrich, R., \& Gazzaniga, M. S. (1997). Islands of residual vision in hemianopic patients. Journal of Comparative Neuroscience, 9, 203-221.

Weiskrantz, L. (1987). Residual vision in a scotoma. A follow-up study of 'form' discrimination. Brain. 110, 77-92.

Weiskrantz, L., Warrington, E. K., Sanders, M. D., \& Marshall, J. (1974). Visual capacity in the hemianopic field following a restricted occipital ablation. Brain, 97, 709-728.

Weiskrantz, L., Barbur, J. L., \& Sahraie, A. (1995). Parameters affecting conscious versus unconscious visual discrimination in a patient with damage to the visual cortex (V1). Proceedings of the Natural Academy of Science USA, 92, 6122-6126.

Yukie, M., \& Iwai, E. (1981). Direct projection from the dorsal lateral geniculate nucleus to the prestriate cortex in macaque monkeys. Journal of Comparative Neurology. 291, 8197.

Zeki, S. (1974). In R. G. E. G. Bellairs, Essays on the nervous system - A Festschrit for Professor J.Z. Young (pp. 327-343). Oxford: Clarendon Press.

Zeki, S. (1991). Cerebral akinetopsia (visual motion blindness): a review. Brain, 114, 811824.
Zeki, S., \& ffytche, D. H. (1998). The Riddoch syndrome: insights into the neurobiology of conscious vision. Brain, 121, 25-45.

Zeki, S., McKeefry, D. J., Bartels, A., \& Frackowiak, R. S. (1998). Has a new color area been discovered? Nature Neuroscience, 1. 335-336.

Zilles, K., \& Clarke, S. (1997). Architecture, connectivity, and transmitter receptors of human extrastriate visual cortex: comparison with nonhuman primates. In K. S. Rockland. J. H. Kaas, \& A. Peters. Cerebral cortex, vol 12 (pp. 673-742). New York: Plenum Press.

\section{Acknowledgements}

It is a pleasure to thank FS and GY for participation in this study. We thank Claudia I. Goebel for her help in performing this study and Niko Kriegeskorte for helpful comments on the manuscript. This work was supported by the Max Planck Society and a HumanCapital and Mobility network grant from the European Community. 


\section{Chapter 4}

\section{Integration of multiple motion vectors over space:}

An fMRI study of transparent motion perception

This chapter corresponds to:

Lars Muckli ${ }^{1}$, Wolf Singer ${ }^{1}$, Friedhelm E. Zanella ${ }^{2}$ and Rainer Goebel ${ }^{3}$ (2002). Integration of multiple motion vectors over space: An fMRI study of transparent motion perception. Neurolmage, in press.

${ }^{1}$ Max Planck Institute for Brain Research, Neurophysiology. Frankfurt am Main, Germany. ${ }^{2}$ Department of Neuroradiology, Johann Wolfgang Goethe-Universitat. Frankfurt am Main, Germany. ${ }^{3}$ Department of Psychology. Neurocognition, Universiteit Maastricht. Maastricht. The Netherlands. 


\section{Abstract}

Visual scenes are frequently composed of objects that move in different directions. To segment such scenes into distinct objects or image planes, local motion cues have to be evaluated and integrated according to criteria of global coherence. When several populations of coherently moving random dots penetrate each other, the visual system tends to assign them to different planes - perceived as transparent motion. This process of integration was studied by changing the angle of motion trajectories with which groups of dots penetrate each other or by varying the spatial constellation of dots moving in opponent directions. Psychophysical testing revealed that stimuli providing almost identical local motion cues could be perceived in three very different ways: 1.) as a matrix of stationary flickering dots 2.) as a single surface of coherently moving dots and 3.) as two transparent dot matrices moving in different directions. Behaviorally controlled functional magnetic resonance imaging (fMRI) was used to identify brain regions that contribute to the integration of local motion cues into coherently moving surfaces. Activation of the human motion complex ( $\mathrm{hMT}+\mathrm{N} 5$ ) and of areas in the fusiform gyrus (FG) as well as in the intraparietal sulcus (IPS-occ) was correlated with the perception of coherent motion and especially $\mathrm{hMT}+\mathrm{N} 5$ took a central role in differentiating transparent motion from single-surface coherent motion. 


\section{Introduction}

Coherent movement of random dots is commonly perceived as motion of a single textured surface. If two superimposed random dot patterns move in different directions, the visual system represents two surfaces (Wallach and O'Connell, 1953). Surfaces that share the same region of visual space without occluding one another are consistently perceived as two transparent surfaces, each defined by a set of dots, moving continuously and independently across each other (Gibson et al., 1959). If additional segmentation cues are available, i.e. contrast, color or binocular disparity (Adelson and Movshon, 1982; Krauskopf and Farell, 1990; Qian, 1997), it is possible to perceive several transparent surfaces in the same region of the visual field. However, in the typical laboratory condition where coherently moving populations of randomly spaced dots are used, no more than two moving surfaces can be perceived simultaneously (Andersen, 1989; Mulligan, 1992). In order to evoke the percept of transparent motion, coherently moving dots need to be bound together perceptually and assigned to the same surface, implying segregation of dots that move in different directions, even if they are closely spaced. Yet this mechanism gets compromised if every dot that moves in one direction is precisely paired for the time of movement by a nearby dot that is moving in the opposite direction (Qian et al., 1994a). In such paired conditions perception of coherent motion vanishes for either set of dots. This effect has been used to study the visual processes that enable transparent motion perception, and it has been used to identify transparent motion sensitive neurons in the monkey visual cortex (Qian and Andersen, 1994; Heeger et al., 1999). Responses of the neurons that are involved in segregating different motion vectors must not be compromised by other nearby dots that move in the opposite direction. In other words, the receptive fields of the neurons evaluating the motion cues must be smaller than the distance between oppositely moving dots so that their responses do not get antagonized. Direction selective cells with these properties have been found in the primary visual cortex (area V1) of monkeys (Snowden et al., 1991; Qian and Andersen, 1994). Surprisingly, however, neurons in $\mathrm{V}_{1}$ preserve uncompromised responses even at dot distances that are so small that transparent motion is no longer perceived. This indicates that the binding process that mediates the association of coherently moving dots to distinct surfaces operates at a different spatial scale i. e. with lower spatial resolution than the segregation process that extracts the motion cues, suggesting that the binding function is achieved by another cortical area. Since the responses of neurons in monkey MTN5 have been shown to change when modifications in the distance between oppositely moving dots lead to perceptual changes (Qian and Andersen, 1994; Heeger et al., 1999), it is likely that the binding function is realized in this cortical area.

Our study is designed to investigate in detail, which areas participate in the cortical network that is responsible for this type of motion integration. Two parameters in the dot displays were systematically varied in order to induce different percepts on the basis of almost identical local motion cues. The orientation of motion trajectories was varied in order to induce either the perception of one coherently moving surface or two transparently moving surfaces. Small orientation differences led to the percept of coherent motion. large differences to transparent motion. The vicinity of oppositely moving dots was varied to induce the percept of either transparent motion, or the perception of a non-moving pattern.

Perceptual thresholds were obtained prior to the AMRI experiments, and were in addition controlled during one of the AMRI experi- 


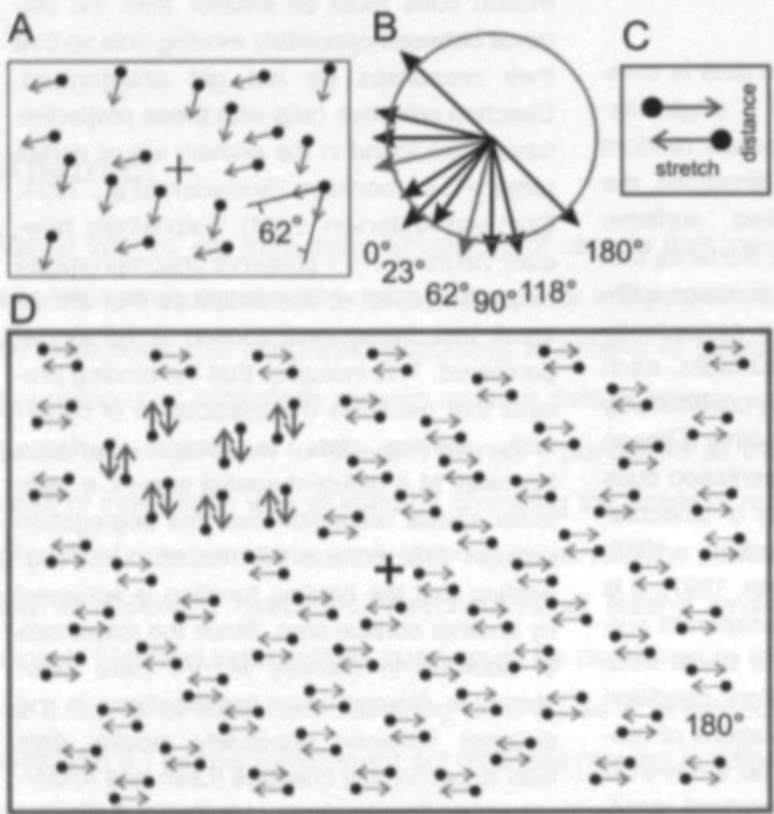

Fig. 1: Transparent motion stimuli consist of two groups of random dot patterns that move in different directions. For the example shown in (A). the motion trajectories of the two groups differs by an angle of $62^{\circ}$. (C) In the fMRI-a experiment 6 different motion angles of motion trajectories were used $\left(0^{\circ}, 23^{\circ}, 62^{\circ}, 90^{\circ}\right.$, $118^{\circ}$, and $180^{\circ}$ ). The motion trajectories were oriented around the left downwards diagonal and resulted in approximately the same velocities (indicated by the length of the arrows). (D) Opponent moving dot displays consisted of randomly distributed pairs of dots that moved in opposite directions. The stimulus is a variation of the 'paired' transparent motion display used by Kolb and Braun (1995) and Qian et al. (1994a). Target regions are defined by the contrast in motion direction. Dots within the target region (shown in the left upper quadrant) move along trajectories that are orthogonal to the surrounding background. (C) The perception of the display depends on the vicinity of dots moving in opponent directions. Vicinity can be varied along the motion direction (stretch) and orthogonal to the motion direction (distance). Both of these parameters were varied during psychometric testing. Only the distance was changed during the IMRI scanning

ments. Blood oxygenation level dependent (BOLD) changes were related to changes in the perceptual condition. Brain activity related to transparent motion perception was crossvalidated under passive viewing conditions.

\section{Materials and methods}

\section{Subjects}

Two psychophysical experiments (PI and PII) and two fMRI experiments (MRI-a and AMRlb) were performed. Ten subjects (eight males, two females, age range: $25-35$ years) participated in the psychophysical studies (PI: 4; PIt: 6) and fourteen subjects (eight males, six females, age range: 25 - 35 years) in the functional imaging studies (seven each fMRI experiment). Four subjects that participated in the fMRI-b participated also in the psychophysical studies (PI: 2: PII: 2). All subjects had normal or corrected to normal vision, had no neurological history and had given their informed consent.

\section{Viewing conditions}

The stimuli were computer generated (Digital DECpc Celebris XL 590, Digital Equipment. Munich, Germany) using a special graphics adapter (ELSA Winner 2000 Pro $X$ ) and the Elsa Powerlib C library. In the AMRI experiments stimuli were projected onto a frosted screen with an LCD projector (EIKI LC-6000, 
Eiki GmbH, Idstein, Germany; graphic resolution $640 \times 480$ pixels; vertical refresh rate 60 $\mathrm{Hz}$ ). Subjects viewed the screen through a mirror mounted to the head coil. The visual field size was $17^{\circ} \times 12^{\circ}$ ( $\mathrm{fMRl-a)}$ and $29^{\circ} \times$ $22^{\circ}$ (IMRI-b). The dot size was $2^{\prime} 50^{\prime \prime}$ ( $\mathrm{fMRI}-\mathrm{a}$ and $\mathrm{MRI}-\mathrm{b})$.

Psychophysical experiments (PI and PII) were similar in most respects but the stimuli were presented with different graphical resolutions. The viewing conditions of the PI experiment (viewing distance: $0.75 \mathrm{~m}$ ) and the AMRI-b experiment (viewing distance 1.30 m) were matched precisely to the same stimulus size and spatio-temporal dot parameters (field size: $29^{\circ} \times 22^{\circ}$, dot size: $2^{\prime} 50^{\prime \prime}$, dot velocity: $2.8^{\circ} \sec -1$ ). In the $\mathrm{PI}$ experiment a low graphic resolution mode was used to deliver the stimuli $(640 \times 480$ pixels; vertical refresh rate: $60 \mathrm{~Hz} ; 21$ inch monitor, Hitachi Accuvue, HM 4921-D). The PII experiment was designed to test the stimuli on a finer spatial scale. Therefore the graphical resolution and the viewing distance were increased for PII (1152 x 864 pixels; refresh rate: 130 $\mathrm{Hz}$, viewing distance $2 \mathrm{~m}$, same monitor) so that the size of the stimuli and their components were markedly reduced (visual field size: $10^{\circ} \times 10^{\circ}$, dot size: $40^{\circ}$, dot velocity: $2^{\circ}$ $\sec -1$ ).

\section{Stimuli}

Stimuli consisted of randomly distributed pairs of dots. In the fMRl-a experiment the distances between the paired dots were kept constant ( 54 ) but the angle between the dots of a pair was varied (see below, and Fig 1ab). No behavioral responses were requested during fMRI-a. In PI, PII, and IMRI-b the dots of a pair moved in opposite directions and contained a target region. Target regions were located in one of the four quadrants of the stimuli (Fig 1d) and were defined by a contrast of motion directions. Dots within the target region moved along trajectories orthogonal to those of the background. The subjects ability to locate the target was examined as a function of the distance between the paired dots.

\section{Stimuli for fMRI-A}

In the fMRI-a experiment the distance between paired moving dots was kept constant but the angles between the motion trajectories of dot pairs were varied in a parametric design. Stimuli consisted of 24 pairs of moving white dots (dot pair density: $0.5 /$ deg2). The distance and the stretch of paired moving dots were set to a constant value of $54^{\circ}$. Angles varied between $0^{\circ}, 23^{\circ}$, $62^{\circ}, 90^{\circ}, 118^{\circ}$, and $180^{\circ}$. The two motion trajectories were oriented symmetrically around the left downwards diagonal (polar angle: p3/4; see Fig. 1a-b). These angles were selected because they could be generated on a computer screen with pixel wise displacements that resulted in approximately the same velocities: $11.8^{\circ}, 10.1^{\circ}, 11.5^{\circ}, 11.2^{\circ}$, $11.5^{\circ}, 11.8^{\circ} / \mathrm{s}$. Note that the two simultaneously presented motion fieids always moved with the same speed and only varied between trials with respect to the angle of the motion trajectories. Blocks lasted for 16 seconds and were presented interleaved with displays of stationary random dot patterns (48 dots) or fixation. The protocol consisted of three repetitions of a $16 \mathrm{sec}$ long sequence consisting of: fixation, static, TM- $0^{\circ}$, static,

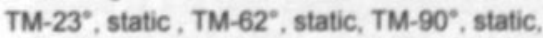
$\mathrm{TM}-118^{\circ}$, static, $\mathrm{TM}-180^{\circ}$, static, fixation. Subjects performed two runs of the same protocol with fixation conditions replacing the static ones in one half of the runs.

\section{Stimuli for P I and P II}

Pairs of white dots move on dark backgrounds ( $\mathrm{Pl}: 300$ pairs, dot pair density $0.5 /$ $\mathrm{deg}^{2}$ / PII: 500 pairs, dot pair density: $5 / \mathrm{deg}^{2}$ ). 


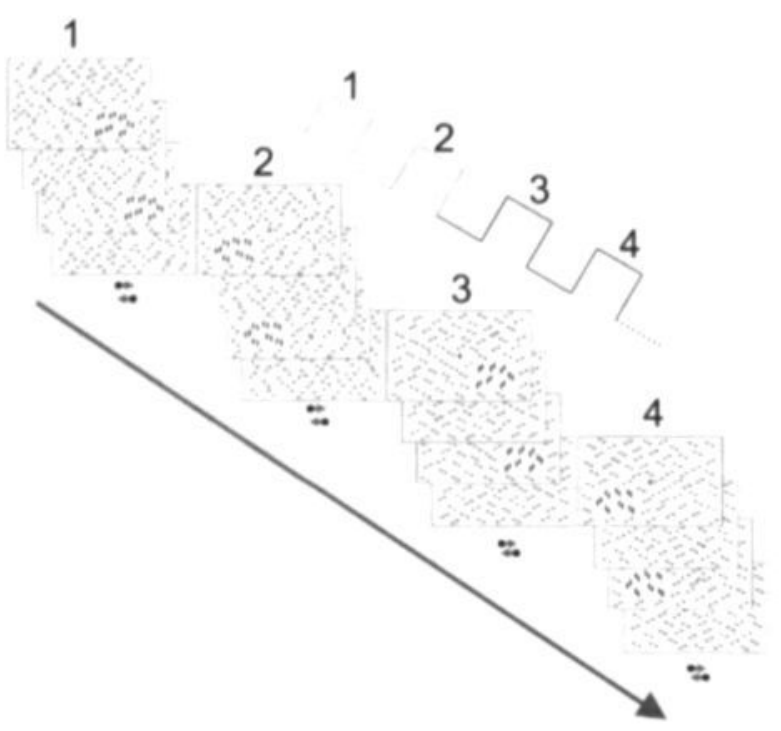

Figure 2: Procedure of fMRI-b experiment. Experimental conditions change after blocks of 32 seconds between opponent motion conditions (1, 2, 3, 4) and fixation conditions. Displays of $\mathrm{TM}(1,2)$ and $\mathrm{NT}(3,4)$ are identical in local motion vectors but the distance of opponent moving vectors is reduced in the NT conditions. Targets appeared and disappeared at random intervals in the left or right lower visual field with a strong tendency $(85 \%)$ to one hemifield. The preferred hemifield changed between blocks of presentation (left in 1 and 3 ; right in 2 and 4). The AMRI-b experiment consisted of four repetitions of this sequence.
Each pair consisted of two nearby dots that appeared simultaneously. moved towards one another, passed each other and disappeared. In the next frame, the pair was presented at another randomly selected screen position, starting the same sequence.

The perception of motion transparency depends on the spatio-temporal relation between the oppositely moving dots (Qian et al., 1994a). We varied two spatio-temporal stimulus parameters: the stretch along the motion direction (others refer to it as dot-lifetime; see Qian et al., 1994a; Kolb and Braun, 1995) and the distance of dots orthogonal to the motion direction (Fig 1c). Stretch and distance are combined to a vicinity ratio, the ratio of dot distance over dot stretch, which will be used below as a measure to account for both parameters simultaneously. For each experiment the stretch was varied in five steps and ranged over both experiments between $2.1^{\prime}$ and $59^{\prime}\left(\mathrm{PI}: 8.4{ }^{\prime}, 14^{\prime}, 19.6{ }^{\prime}\right.$, 25.2', 58.8' / PII: 2.1', 3.5', 4.9', 6.3, 14.77)
The distance ranged over both experiments between $0.7^{\prime}$ and $25^{\prime}$ ( $\mathrm{PI}: 2.8^{\prime}, 5.6,8.4^{\prime}, 14^{\prime}$, 25.2' / PII: $0.7,1.4^{\prime}, 2.1^{\prime}, 3.5^{\prime}, 6.3^{\prime}$ ). Target regions (size PI: $4.4^{\circ} \times 5.3^{\circ} / \mathrm{PII}: 2^{\circ} \times 2^{\circ}$ ) were centered in one of the four quadrants of the display (vertical eccentricity PI: $4.4^{\circ} / \mathrm{PII}$ : 1.5 $5^{\circ}$, horizontal eccentricities: $\mathrm{PI}: 1.1 \% \mathrm{PII}$ $1.5^{\circ}$ ). In PI the motion trajectories were oriented along the main axes in PII along the main diagonals.

\section{Stimuli for fMRI-B}

Two stimulus conditions from experiment PI were used for fMRI-b measurements. The stimuli consisted of 300 pairs of oppositely moving white dots (visual field size $29^{\circ} \times 22^{\circ}$. dot size: $2^{\prime} 50^{\prime \prime}$, dot velocity: $2.8^{\circ} \mathrm{sec}$, dot pair density: $0.5 / \operatorname{deg} 2$ ). The stretch was kept constant at a value of 19.6', which led also to a constant duty cycle of $8.3 \mathrm{~Hz}$. Distance was varied between two values, $8^{\prime}$ and $25^{\circ}$. From the PI experiment it was evident that this change in distance would suffice to create two distinct conditions of opponent motion. 
With a distance value of $25^{\circ}$ displays were perceived as transparently moving dot displays (TM), while with a distance of 8 'displays were perceived as static incoherent flicker (non transparent: NT). In the latter displays, targets defined by motion contrasts were not detected.

During fMRI scanning TM and NT displays were presented for blocks of 32 seconds (equal to 8 scans of a brain volume covered by 15 slices). Within a block, targets appeared and disappeared at random intervals (mean inter target interval: $2.5 \mathrm{sec}$; mean target presentation time $3.5 \mathrm{sec}$ ). The time required for a build up of a target was $120 \mathrm{~ms}$, as it takes the duration of a dot life cycle until every dot pair has been exchanged. Targets appeared randomised in the left or right lower visual field with a strong bias $(85 \%)$ towards one hemifield. The preferred hemifield changed between blocks of presentation. Conditions with preferred left or right target presentation and TM or NT conditions were alternated and presented interleaved with periods of fixation. A fixation cross was present during all conditions and was the only stimulus during the fixation condition. (Protocol consisted of four repetitions of: TM-left, TM-right, NT-right, NT-left; see Fig. 2)

\section{The task}

In the fMRI-a experiment subjects were instructed to attend to the stimulus while maintaining fixation on a small white cross in the center of the screen.

In the psychophysical experiments stimuli were presented for $250 \mathrm{~ms}$. Subjects were asked to guess the location of the target in one of four quadrants of the display (fouralternative forced-choice testing, subjects entered their selection with the right hand on a number key pad in which the position of the numbers matched analogously to the position of the targets: ' 4 ' left-upper, ' 5 ' 'right-upper.
'2' right-lower, '1':left-lower) and rate their confidence subsequently using categories one to four (indicated by pressing one of four adjacent keys accessible with the left hand on a German PC keyboard: ' $y$ ', ' 'x', 'c', 'v'). At least 870 trials were performed by each subject. A small number of catch trials were presented in some selected conditions. Catch trials contained no targets and allowed the assessment of the baseline for confidence ratings.

In the fMRI-b experiment subjects were instructed to maintain fixation on a small white cross, which was present in the center of the screen throughout all conditions, and to respond to every onset of a target by indicating its location with the left or right response button (for left and right target presentation). Offset of the target was indicated by releasing the response button. Fixation was controlled during the experiment in two subjects using a MRI compatible infrared eye-recording device (Ober2, Permobil Meditech AB, Timrá, Sweden).

\section{Image acquisition}

Echoplanar images were collected on an 1.5T scanner (Siemens Magnetom Vision, Siemens, Erlangen, Germany) using the standard head coil and the Siemens Magnetom gradient overdrive. We used a gradient echo echoplanar sequence $(\mathrm{TR}=4000 \mathrm{~ms}$; $\mathrm{TE}=$ $69 \mathrm{~ms}$; $F A=90^{\circ}$ ) to visualize changes of BOLD (blood oxygen level dependent) contrast $\left(\right.$ FOV $=200-220 \mathrm{~mm}^{2}$, slice thickness = $4 \mathrm{~mm}$, imaging matrix $=128 \times 128$; resulting voxel size $=$ ca. $1.6 \times 1.6 \times 4 \mathrm{~mm}^{3}$ ). Images were acquired in 15 contiguous slices oriented approximately in parallel to the calcarine fissure. A T1-weighted 3D magnetization prepared rapid acquisition gradient echo sequence (MP RAGE) scan (voxel size $=1.0$ $\times 1.0 \times 1.0 \mathrm{~mm}^{3}$ ) lasting $8 \mathrm{~min}$ was recorded in the same session as the functional measurements. Additional T1-weighted 3D data sets tuned to optimize the contrast between 
gray and white matter were recorded in separate recording sessions lasting $24 \mathrm{~min}$.

\section{Data analysis}

\section{Analysis of psychophysical data}

Performance in target location and subjective confidence ratings were correlated for each subject and each stimulus condition. Performance and confidence ratings were plotted as a function of stimulus conditions. Stimulus conditions were expressed as the ratio of motion stretch over dot distance. Individual psychophysical data were incorporated in a group analysis.

During fMRI-b experiment responses were considered to be correct if the subjects indicated the correct side of the target (left or right) within 3 seconds after appearance and with release of the button within 3 seconds after disappearance of the target. $100 \%$ performance would include the correct detection of all on- and offsets. The chance level for onset detection is $25 \%$ correct onset responses (leaving the offset response unconsidered).

\section{fMRI data analysis}

Data analysis including preprocessing (3Dmotion correction, spatial and temporal smoothing, linear trend removal), correlation analyses (using the general linear model for multiple regressions), 3D-transformation into the coordinate system of Talairach and Tournaux (1988), surface rendering, cortex inflation, and cortex flattening were performed using BrainVoyager 4.4 software, which is described in more detail elsewhere (Dierks et al., 1999; Kriegeskorte and Goebel, 2001; Goebel et al., 1998; Linden et al., 1999)

Prior to statistical analysis functional images were temporally smoothed using a FFT based band-pass filter. 2D images were then transformed into the $3 \mathrm{D}$ structural data set and interpolated to the same resolution $\left(\right.$ voxel size $\left.=1.0 \times 1.0 \times 1.0 \mathrm{~mm}^{3}\right)$. Since the 2D functional maps and $3 D$ structural measurements were performed within the same recording session, coregistration of the respective data sets could be computed directly relating the Siemens slice position parameters of the T2* weighted images and the T1-weighted 3D MP RAGE measurements to the initial overview (scout). For each subject the structural $3 D$ and functional $4 D$ data sets were transformed into Talairach space (Talairach and Tournaux, 1988) which allows to compare activated brain regions across different experiments and across different subjects and to determine Talairach coordinates of activated regions. The Talairach transformation was performed using the manually specified location of the anterior and the posterior commissure (AC, PC, to align the stereotaxic axes) and the extreme points of the cerebrum to perform a piecewise affine and continuous transformation for each of the twelve defined subvolumes. The transformed functional data were then smoothed in 3D space with a Gaussian filter (FWHM $=3 \mathrm{~mm}$ ) and corrected for head movements with subvoxel precision (translation and rotation parameters of rigid body transformations relative to third volume). Movement corrections were less than 0.25 $\mathrm{mm}$ in all subjects.

In order to evaluate statistically the differences between the experimental conditions we used a multiple regression approach. In the fMRI-a experiment the stimulation protocols for the different motion trajectories were used in a six-predictor model (TM- $0^{\circ}$, TM$23^{\circ}, \mathrm{TM}-62^{\circ}, \mathrm{TM}-90^{\circ}, \mathrm{TM}-118^{\circ}, \mathrm{TM}-180^{\circ}$ ). Each stimulation protocol served to obtain appropriate reference functions reflecting experimental and baseline conditions, respectively (experimental condition $=1$. 
baseline condition $=0$ ). The stimulation protocols were convoluted with a hemodynamic response function (Boynton et al.. 1996) to account for the expected delay and devolution of the BOLD signal. These reference functions served as independent predictors for a general linear model (GLM). At each location (106720 voxels in Talairach space). the amount of explained variance was tested for significance using F- statistics. P-values were corrected for multiple comparisons using Bonferroni correction over all (106720) voxels. Only Bonferroni corrected P-values are indicated as corrected. For statistical comparisons between the experimental conditions the mean value for each condition was approximated by the beta weights of the GLM model. For the analysis of systematic changes in relation to the increasing angles in fMRI-a experiment, beta weights were correlated to the angles of the TM conditions ( $r$-, and $\mathrm{p}$ - values). Contrast maps were calculated on the basis of the GLM model (beta weights) and tested for significance using tstatistics.

In the fMRI-b experiment the stimulation protocol for TM and NT served to obtain appropriate reference functions. These reference functions served as independent predictors for a general linear model (GLM). The proportion of variance explained by the model was tested for significance using F-statistics (ANOVA). If $p$-values are indicated to be corrected for multiple comparisons, a Bonferroni correction over all 106720 voxels has been performed. Contrast maps were calculated by comparison of the estimated beta-weights and are tested for significance by t-statistics.

The same data were also analyzed with a four predictor GLM to account for the left and right target presentation (TM-right, TM-left, NT-right, NT-left). Multiple correlation maps and contrast maps were superimposed on the T1-weighted $3 D$ anatomical reference scans. For the visualization of group data we used the individual MNI template brain (template provided with courtesy of the Montreal Neurological Institute).

The individual brain surfaces and the template brain were reconstructed based on the high-resolution T1-weighted structural $3 \mathrm{D}$ recordings. The white/gray matter boundary was segmented, reconstructed, smoothed, morphed and flattened as described in detail elsewhere (Dierks et al., 1999, Kriegeskorte and Goebel, 2001). A morphed surface always possesses a link to the folded reference mesh so that functional data can be shown at the correct location of partially inflated as well as flattened representations.

For a precise delineation of early visual areas in the single subject analysis, we used retinotopic maps generated in separate experiments (Engel et al., 1994: Goebel et al., 1998; Sereno et al., 1995). Twelve contiguous slices were obtained approximately in parallel to the calcarine fissure (TR $=3000$. voxel size $=1.6 \times 1.6 \times 3 \mathrm{~mm} 3$ ) and subjects were stimulated with slowly expanding checkerboard rings and slowly rotating ray shaped checkerboards from which eccentricity maps and polar angle maps were gained (for details see Goebel et al 1998). From the combination of eccentricity and polar maps we estimated field sign maps as described by Sereno et al. (1995) to delineate early visual areas.

\section{Results}

\section{Psychophysics}

The perception of the dot displays changed substantially when dot distance was varied. Subjective reports and measurements of target detectability indicate that motion transparency is perceived best if dot distance and dot stretch are at the upper end of the tested 
A

B

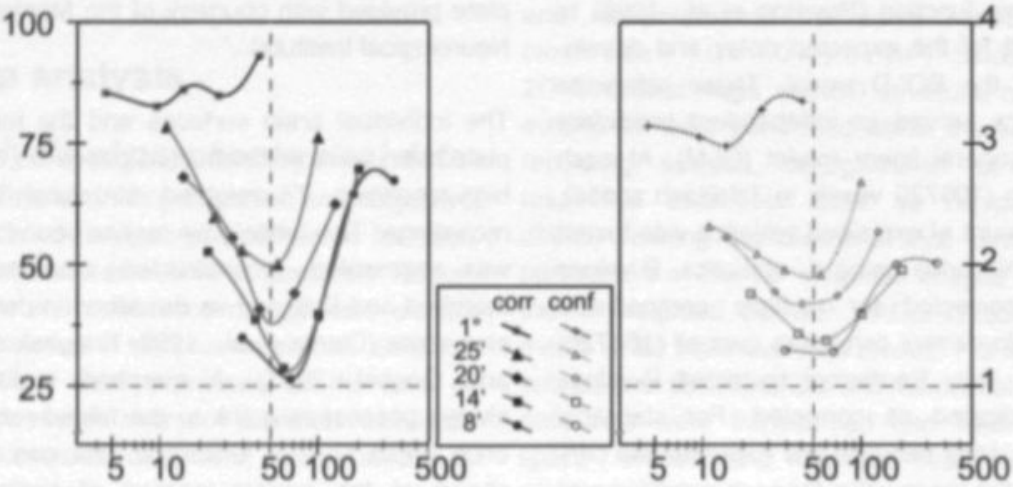

C
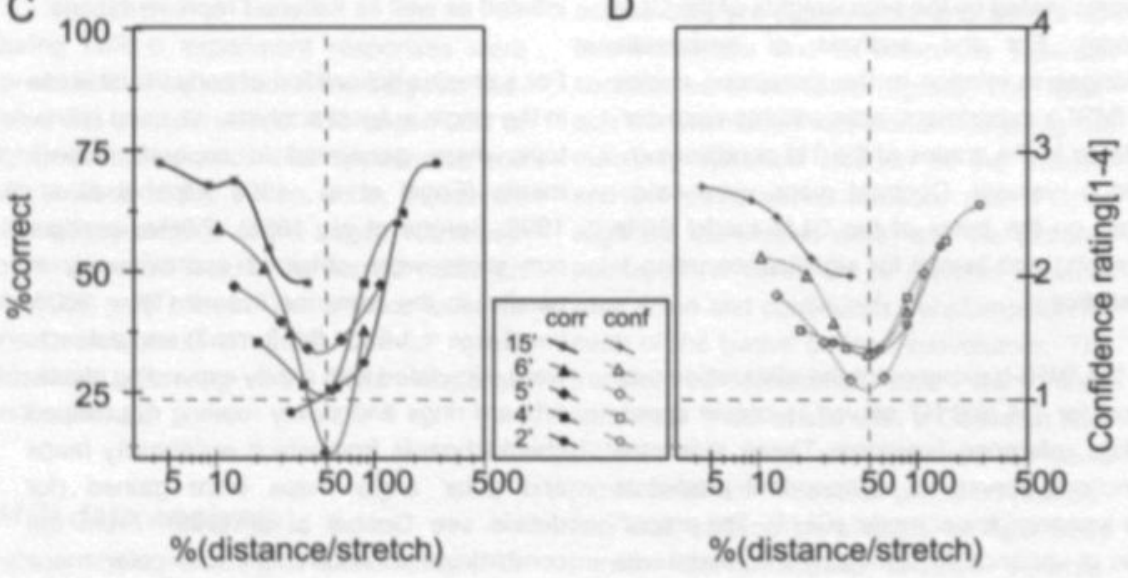

Figure 3. Performance in psychophysical experiment I and II. Behavioral mean responses of 10 subjects (PI: 4; PII: 6) to 50 stimulus conditions (PI: 25; PII: 25). Mean performances for PI (A) and PII (C) are plotted in solid symbols for each stimulus condition. Performances in conditions of equal dot stretch are connected with solid lines that are fitted by a spline function (stiffness: 0.25). Dot vicinity is given on the abscissa expressed in percent ratio of dot distance to motion stretch (vicinity ratio). Mean subjective confidence ratings for PI (B) and PII (D) are plotted in open symbols (and dotted lines) for each stimulus condition and refer to the right ordinate. The minimal and maximal values of left and right ordinate systems were aligned (performance $25 \%$ to $100 \%$ with confidence ratings 1 to 4 ). Confidence ratings were correlated to performance and were significant for all conditions that exceeded the chance level signifcantly (not shown). The stimulus conditions for the MRI-b experiment were selected from the psychophysical functions shown in (A). 
range. Thus, both parameters had to be considered together for quantification of stimulus conditions. When the vicinity ratio, the ratio of dot distance over dot stretch, was smaller than $40 \%$, the dot pairs were perceived as smeared but oriented lines that form a texture and stimuli appeared as non transparent and non moving. Nevertheless, different directions of motion were detected indirectly because they produced smeared lines of different orientation. Thus, target regions popped out due to orientation contrast. Perceptual smearing increased with decreasing dot distance. Accordingly, target detection improved with decreasing dot distances for stimuli with a distance ratio $<40 \%$ (note the descending slopes of solid lines in the left sections of Fig 3a-d).

Target detection was poorest at a vicinity ratio of $50 \%$. In this condition subjects reported to perceive a texture field of incoherently flickering. stationary dots. Confidence ratings (dotted lines in Fig. 3 b, d) and performance (solid lines in Fig. $3 \mathrm{a}$, c) clearly indicate that motion transparency and direction of local motion vectors were neither perceived (confidence rating) nor detected (forced-choice performance). Performance, confidence rating and the correlation between performance and confidence were minimal and not significant $(p>.05$; for all conditions with stretches $<15$ ). Consequently, we used this stimulus configuration as our non transparent control condition (NT) in the subsequent fMRI-b study. (On a long exposure photograph, stimuli with a ratio of $50 \%$ appear as a texture of equally spaced double line segments whose stretch is twice as long as their distance).

When vicinity ratios increase above $80 \%$. performance increases again (compare ascending solid slopes in the right sections of Fig 3 a-d). Dots moving in the same direction are grouped perceptually and separated from nearby dots that move in the opposite direc- tion. Subjects now report to perceive transparent moving surfaces. With increasing vicinity ratios, perception of transparency increases further and opponent moving dots appear as entirely unrelated. To induce a robust percept of transparent motion in the fMRI-b experiment we selected for the transparent motion condition (TM) a vicinity ratio of $130 \%$. We settled for this value because with much higher ratios, e. g. $300 \%$, the texture becomes homogeneous and dots do no longer appear in clusters.

During the fMRI-b experiment subjects responded correctly in $85 \%$ of the TM conditions but performed only at chance level during NT condition. This was consistent with the prior expectation derived from the psychophysical experiments.

In the fMRI-a experiment, subjects reported verbally to have no transparent motion percept in the TM- $0^{\circ}$ and only a weak percept of transparent motion in the TM-23* condition. In all other conditions the percept of transparent moving surfaces was vivid (TM-62", TM$90^{\circ}, \mathrm{TM}-118^{\circ}, \mathrm{TM}-180^{\circ}$ ).

\section{Experiment fMRI-A}

\section{Group analyses}

We found activity of areas $\mathrm{MMT}+\mathrm{N} 5$, right IPS-occN3a, V1N2, and FG-collateral to increase with increasing angles between the two motion trajectories (linear trend contrast: TM $-0^{\circ}<\mathrm{TM}-23^{\circ}<\mathrm{TM}-62^{\circ}<\mathrm{TM}-90^{\circ}<\mathrm{TM}-$ $118^{\circ}<\mathrm{TM}-180^{\circ}$ ). Corresponding positive contrast values are shown in Fig. 4 with redto-yellow colors. The most significant linear trend $(r=.97, p=.002)$ was found in hMT $+N 5$ of the left hemisphere, followed by right IPSocc $(r=96, p=002)$, left IPS-occ $(r=.95$, $p=004)$ and right $h M T+N 5(r=.90, p=02)$. For further details, see Table 1. In the contrary, activity in parietal IPS, left post-IPS-occ. and right dorsal V1N2 decreased with 

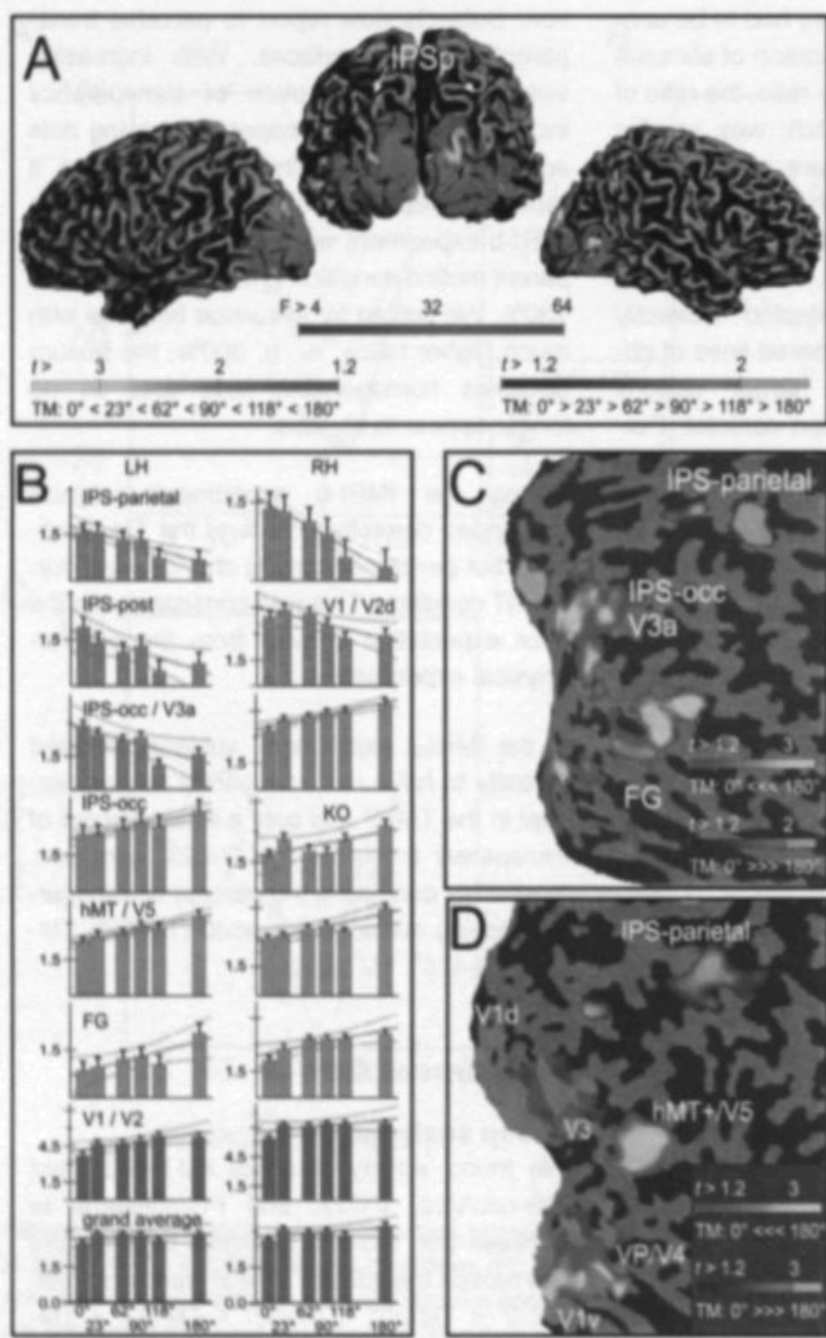

Figure 4. Group results and single subject results of IMRI-a experiment. (A) Three different statistical maps were superimposed on a cortical reconstruction of an MNI-template brain and are shown from three different views (left, posterior, right). Regions contributing significantly to the GLM model are shown In green (F>4, $p<0.0005$, uncorrected). Maps for linear trend contrast were calculated from the GLM. Regions that responded with increasing amplitude for increasing angles between motion trajectories are shown with red-toyellow colors. Regions that respond with decreasing amplitudes for increasing angels are shown with blue-to-cyan colors. (B) Activation profiles for the regions shown in A provided for left (L.H) and right (RH) hemisphere (colors of the profile match to the color of the regions in A). Beta weights were taken from the GLM and used as measure for central tendency. Bars show the beta weights and the standard error for the corresponding conditions: TM-0", TM-23*, TM-62*, TM-90*, TM-118*,

TM-180* Correlation between beta weights and angles are given in Table 1. The regression line and the $95 \%$ confidence interval of the regression (between beta weights and angles) are indicated for each actvation profile in red. (C) Contrast maps of the group data are shown again on an flattened representation of the right cortical sphere and can be compared to single subject data. (D) Single subject contrast maps on flattened representations of the subjects right cortical sheet. Regions that show increasing activity with increasing angels are shown in colors red-to-yellow. Regions that decrease with increasing angles are shown in blue-to-cyan colors. Field sign maps (green and blue) are derived from individual retinotopic mapping and indicate the extensions of the earty visual areas (green: V1, V3, VP. blue. V2, V3a. V4). Gray scale contours reflect the tissue curvature. 


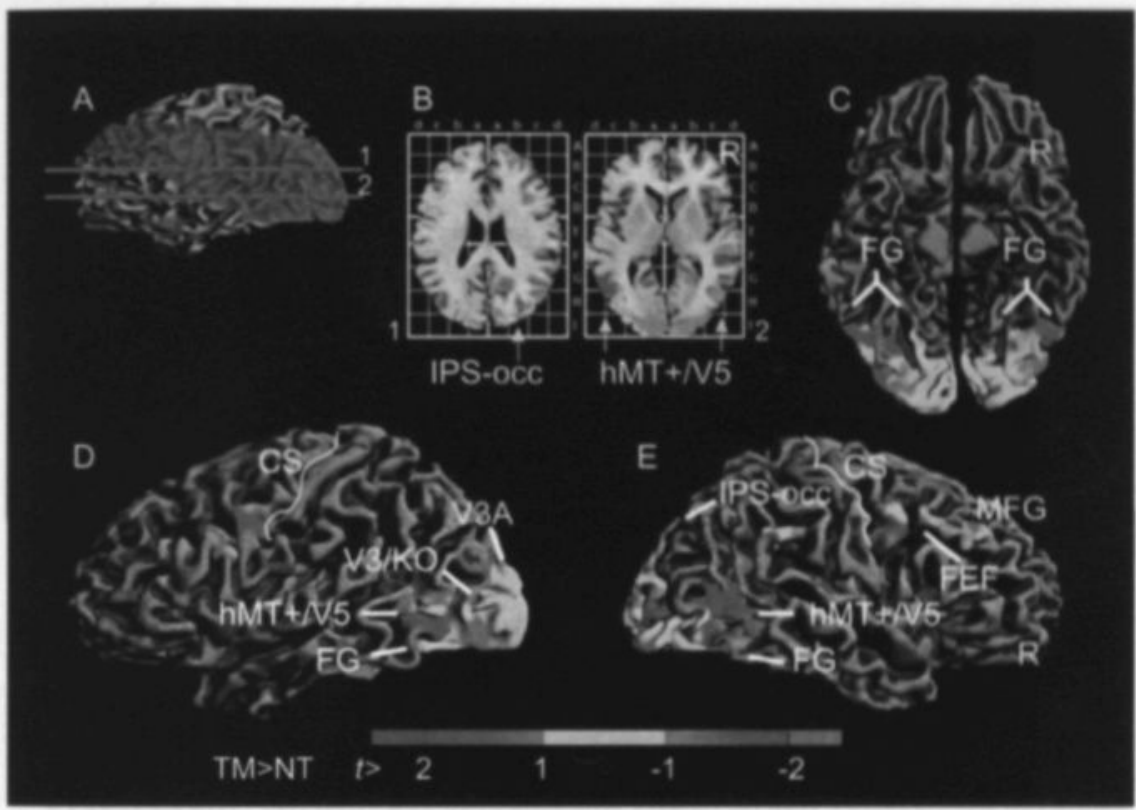

Figure 5. Group analyses of mRI-b experiment. Group results of seven subjects superimposed onto an individual MNI template brain. Multisubject GLM analyses were performed using a GLM with independent predictors for conditions of TM (transparent motion) and NT (non transparent). Colored region contribute significantly to the GLM (F>4; p<0.005; uncorrected). Contrast between TM and NT are indicated on separate color scales: More activation during TM than during NT is shown with redto-yellow colors. More activation during NT than TM is shown with blue-to-cyan colors. Regions with significant contrasts are labeled in the axial slices $(B: z+6 ;+22)$, in the lateral (D, E) and in the ventral views (C) of the brain surface (see Table 2). The analyses was restricted to the brain region that was commonly imaged in all seven subjects (A: shown in blue). The cortex of the template cerebrum was segmented and reconstructed along the gray- white matter border shown in (B) (blue and green).

increasing angels (Fig. 4, blue-to-cyan colors, Table 1). Other occipital regions responded equally well to the different conditions. A grand average of all activated regions ( $F>4$, $p<0.0005$, uncorrected) showed no linear trend (Fig. 4, bottom)

\section{Single subject analyses}

For each subject, seperate contrast maps were calculated for the increase and for the decrease of activity with increasing angle between the two motion trajectories. Subsequently. maps were compared to the area boundaries as shown for one subject in Fig. 4d. Early visual areas (V1, V2, V3, VP, V3a) were delineated by retinotopic mapping of the vertical and horizontal meridians using field sign maps (Engel et al., 1994; Sereno et al., 1995). As in the group analysis, area hMT+1 V5 showed the strongest increase of activity with increasing angle. For the subject shown. the response increase was more widespread in ventral retinotopic visual areas (V1v, V2v, VPN4) than in dorsal early visual areas. A negative linear trend - decreasing activity with increasing angles - was observed in IPSparietal, and posterior IPS. 


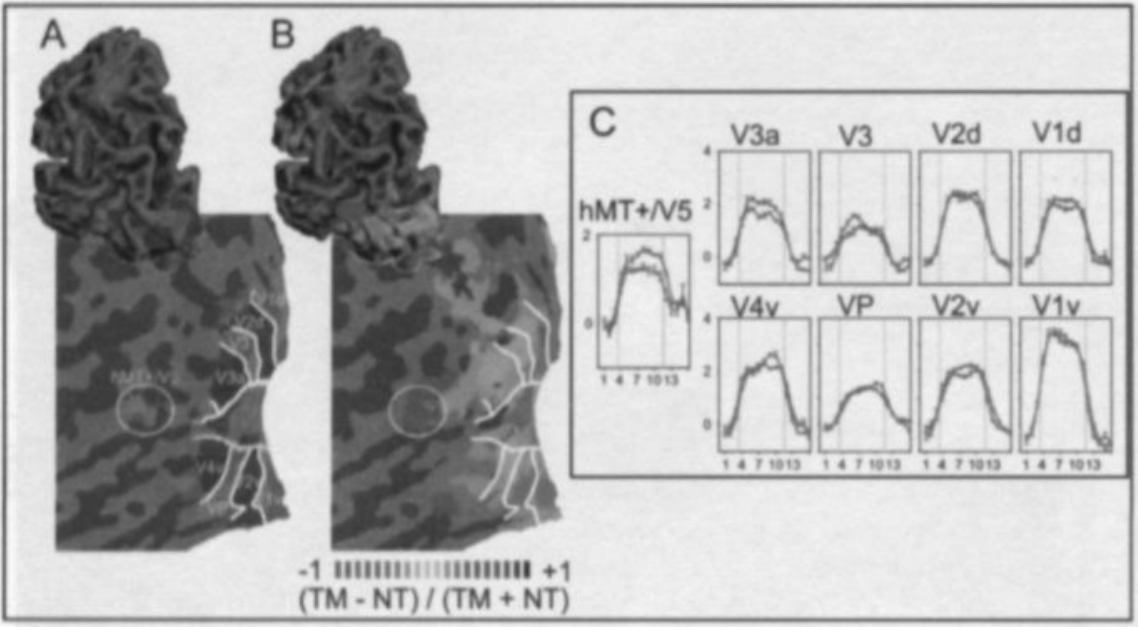

Figure 6. Single subject analyses of MRI-b. Results of retinotopic mapping and mRI-b experiment superimposed on a reconstructed (top) and on a flattened representation of the left occipital cortical sheet. Gray scale contours reflect the tissue curvature (dark concave, light convex). (A) Field sign maps (green-blue) were derived from the eccentricity and polar angle maps and used to delineate early visual areas (see methods). (B) Relative contribution map ([TM - NT] / $[\mathrm{TM}+\mathrm{NT}$ ) indicates if a region is stronger activated by TM stimuli (red) or by NT stimuli (green) or equally by either stimuli (yellow). (C) Averaged signal time course in early visual areas and $\mathrm{hMT}+\mathrm{N5}$ from the subject shown in A and B for TM (red) and NT (green; small whiskers indicate 1 +- standard error of the mean).

\section{Experiment fMRI-b}

\section{Group analyses}

The main results of the group analysis are summarized in Fig. 5 and Table 2 and show regions of enhanced activation across seven subjects. To be considered in further analyses an area had to be correlated significantly with reference functions for either TM (transparent motion) or NT (non transparent) or both (multiple $R>0.4$, $p<0.00001$, corrected) and had to exceed a minimal size of 4 $\times 4 \times 4 \mathrm{~mm} 3$ (Fig. 5). Most of the occipital, the occipito-temporal and occipito-parietal cortex was activated equally during NT and TM conditions (yellow regions in Fig. 5). Differential activation levels under NT and TM conditions were only found in higher motion responsive areas and in somato-motor areas, the trans- parent motion condition being more effective in the human motion complex (hMT+N5). parts of the fusiform gyrus (FG), in the occipital part of the right intraparietal sulcus (IPSocc) and around the central sulcus (red in Fig. 5. Table 2). Weaker activity during transparent motion perception was observed in areas VP I KO and around the precentral I mid-frontal sulcus (green in Fig. 5, Table 2).

Visual areas V1, V2, V3a, KO/LOC, and the FEF were identified according to functional and topological criteria (Sunaert et al., 1999; Tootell et al., 1998b), the other areas were classified only according to anatomical criteria (FG Pre/PostCS, MFG).

Relative modulation of the BOLD signal during $\mathrm{TM}$ was expressed as percent signal change relative to NT and tested explicitly with the contrast TM > NT (see Table 2). The 
amplitude of activation was derived from the beta weights of the group analysis for the selected regions (Table 2). Besides response-related activity in the central sulcus, the highest contrast values were found in left $h M T+N 5$ ( $t=6.3 ; p<.01$, corrected), followed by right $F G(t=6.0 ; p<.01$, corrected $)$ and right $h M T+N 5$ (t=5.6; $p<0.01$, corrected).

\section{Single subject analyses}

A typical activation pattern from one subject is shown in Fig. 6. The largest regions with highest differential responses to TM were the human motion complex ( $\mathrm{MMT}+\mathrm{N} 5$ ), the somatosensory and motor cortex and the frontal eye fields (FEF). Additional regions with less pronounced differential TM activation were observed in the fusiform gyrus, in the right intraparietal sulcus (IPS-occ), and in a subpart of V1. As V1 exhibits slightly inhomogeneous activation in this subject we present two time courses (V1d, V1) that indicate the range of possible profiles. All other subjects showed even less variation in V1. Areas V2, V3 and VP showed also no or only small differences between TM and NT conditions. In this subject only area V3a showed weaker activity during $\mathrm{TM}$. The location of many activated regions could be specified more precisely with regard to retinotopically mapped areas (Fig. 6a). Within the fusiform gyrus (posterior part of the gyrus anterior), a TM sensitive region was found to lay anterior or partly overlapping to area V4v (Lueck et al., 1989; Zeki et al., 1998) and area V8 (Hadjikhani et al., 1998).

IPS-occ is located at the occipital end of the (IPS) were it descends to the superior occipital sulcus (SOS) near the transverse occipital sulcus and anterior to V 3 A. Becasuse of its anatomically location IPS-occ is likely to correspond to area V7 (Tootell et al., 1998b). In this subject - like in three others - only right presumed $V 7$ showed differential TM activa- tion. Of the remaining three subjects, two showed TM-enhancement in presumed $V 7$ in both hemispheres and one only in the lef hemisphere.

\section{Target related activity}

Group data and single subject data were reanalyzed using a four predictor model (GLM). In this approach predictors specified not only the perceptual condition (TM, NT) but also the hemifield of target presentation (left. right). In each block the targets appeared more frequently $(85 \%)$ in the left or right lower visual hemifield. Regions that are responsive to the lower visual field (dorsal retinotopic areas V1d, V2d, V3, V3a, ct. Fig 6c) were inspected closely for signal enhancement during blocks of contralateral target presentations. A left versus right contrast was separately calculated for TM and NT conditions. Neither contrast disclosed an area that exhibited enhanced activation when targets were in the contralateral visual field.

\section{Eye movements and attention shifts}

We monitored the stability of eye fixation during the fMRI-b experiment with an infrared eye-tracker device in two subjects yielding no differences - within a precision of $1^{\circ}$. between fixation and other conditions (TM or NT). Even though covert attention shifts (Petit and Haxby, 1999) might occur during the target search in the AMRI-b experiment, it is unlikely that these contributed to the enhanced activity during $\mathrm{TM}$ because mean search times were longer during NT trials ( $87 \%$ of the trial time) than during the TM triais ( $54 \%$ of the trial time). Mean search times indicate in percent the time in which no button was pressed with respect to trial duration. 


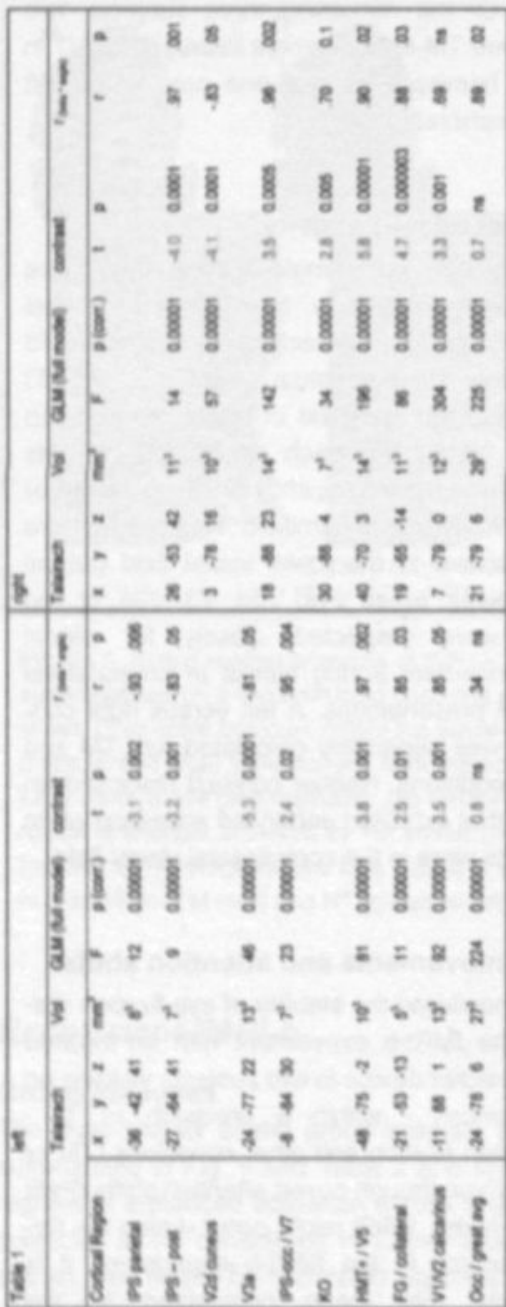

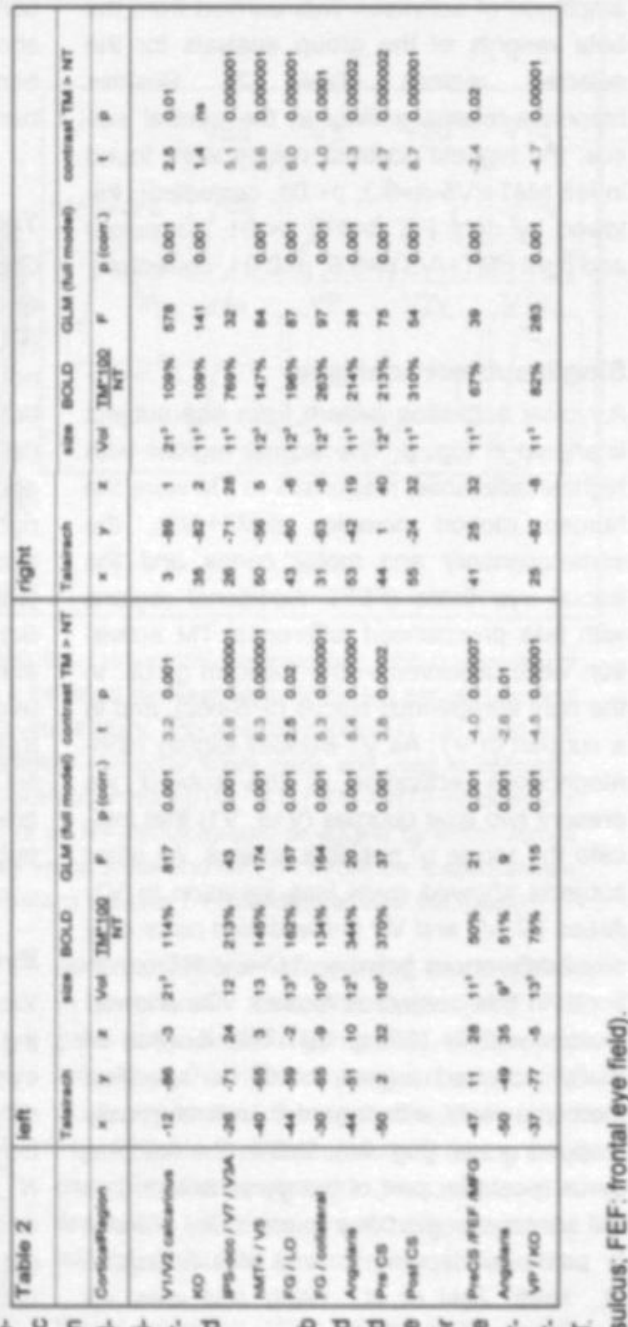

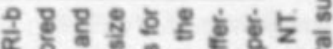

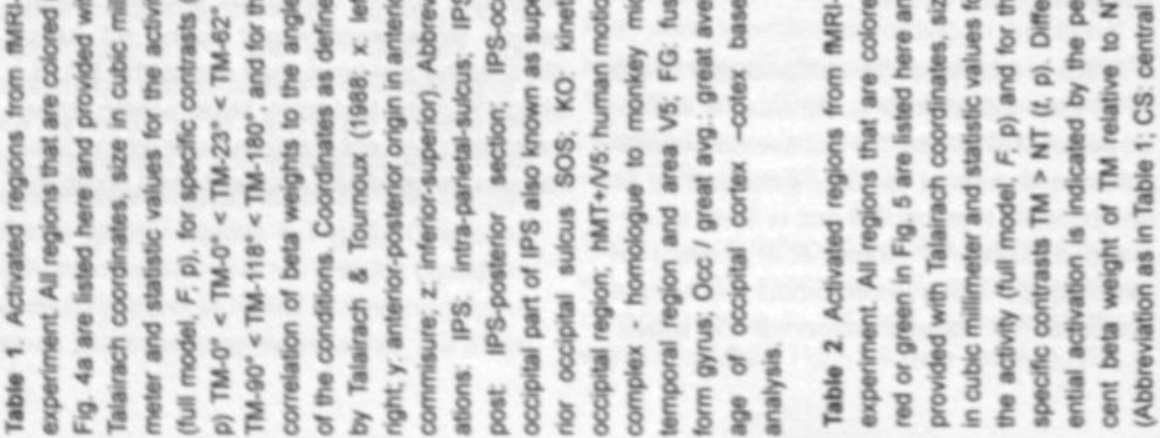

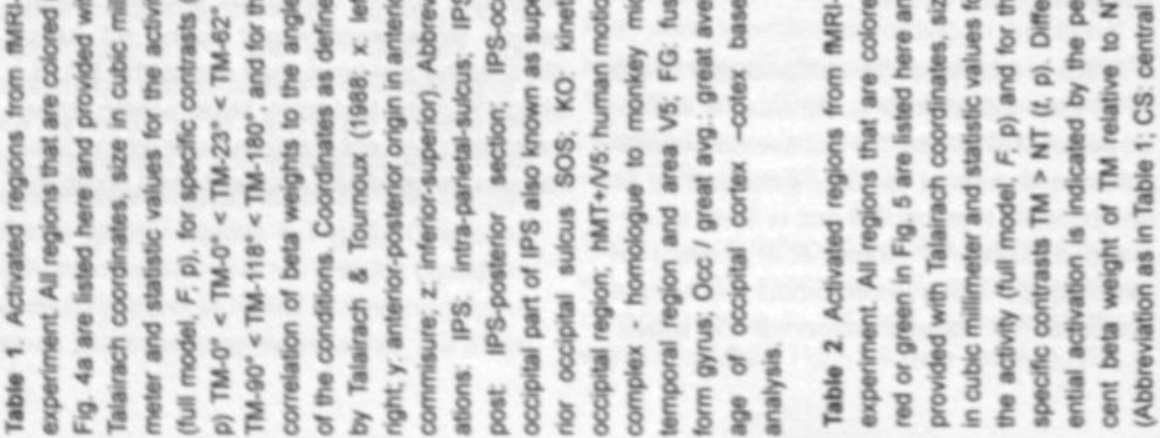

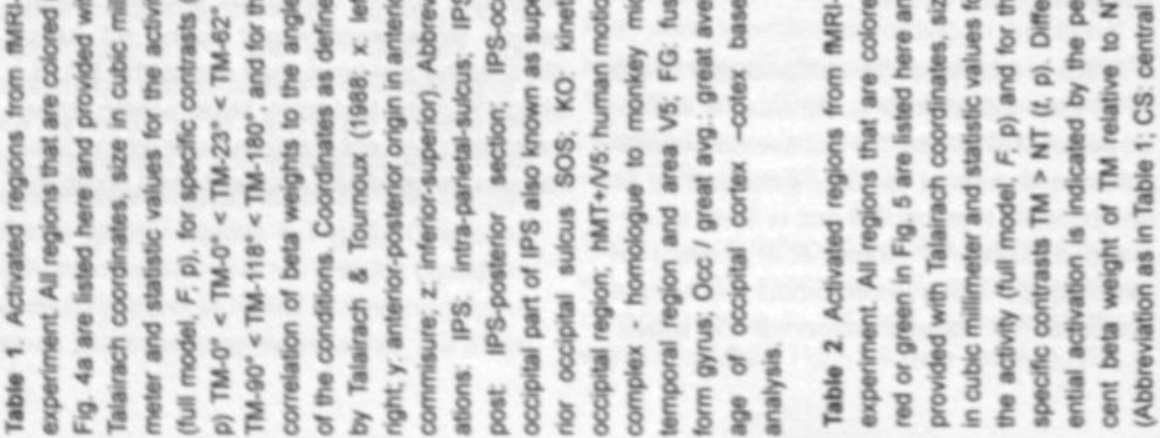

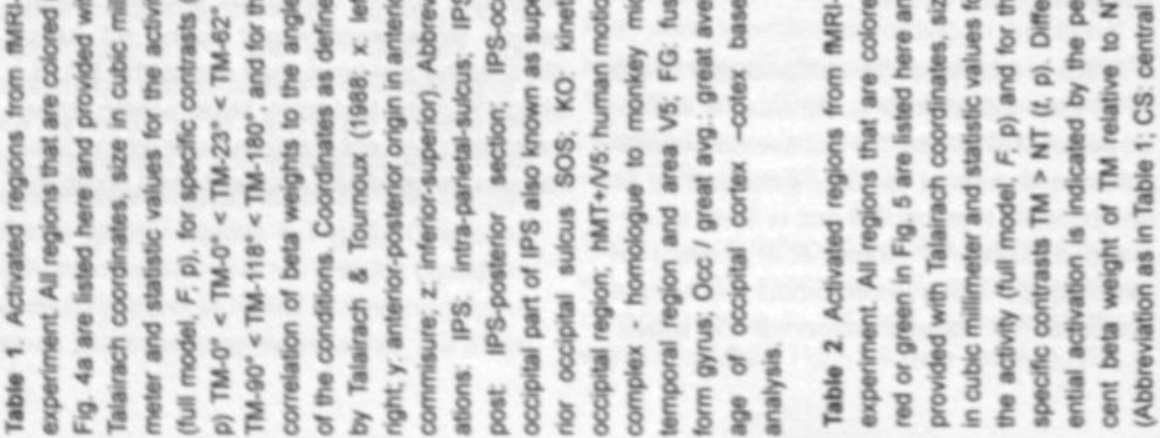

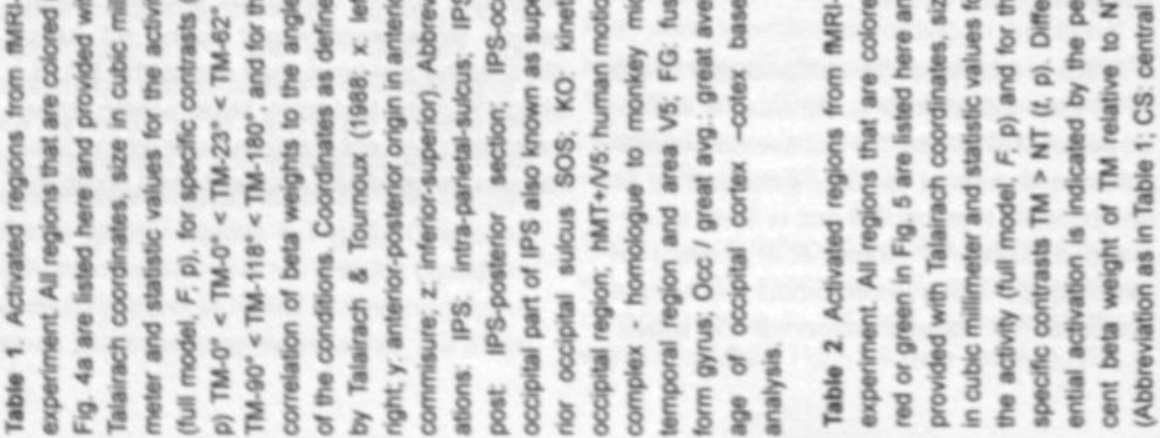

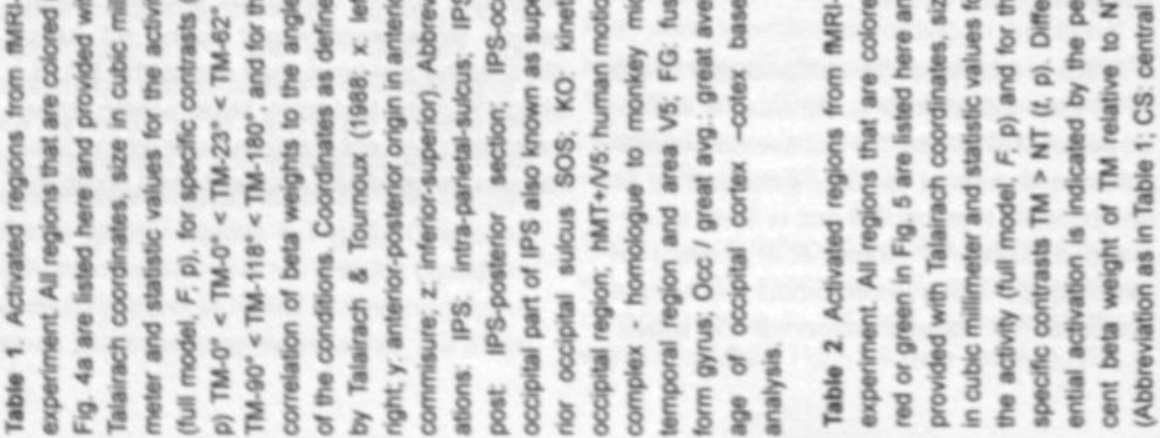

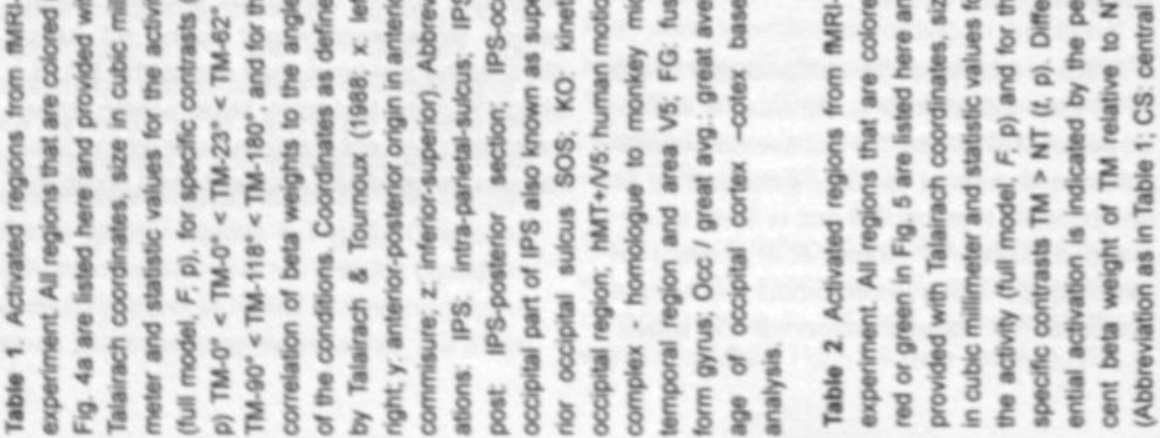

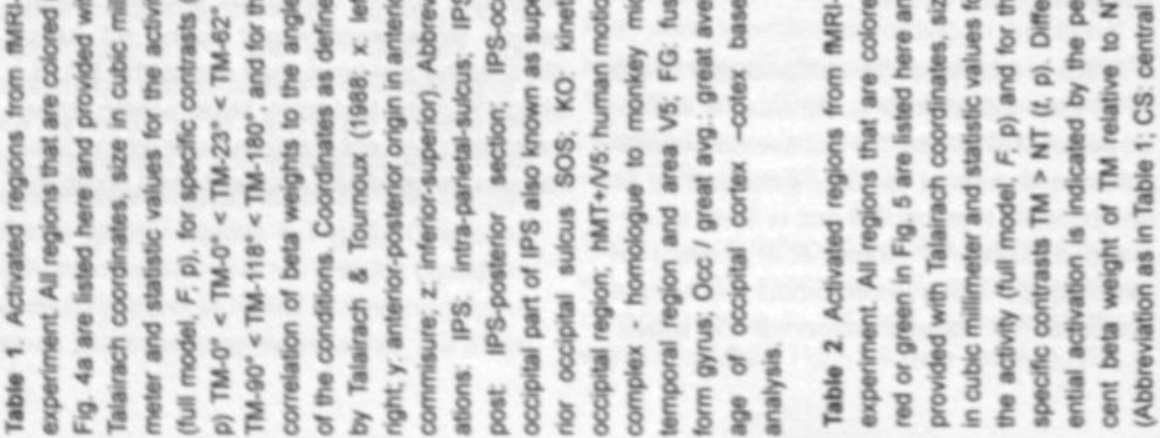

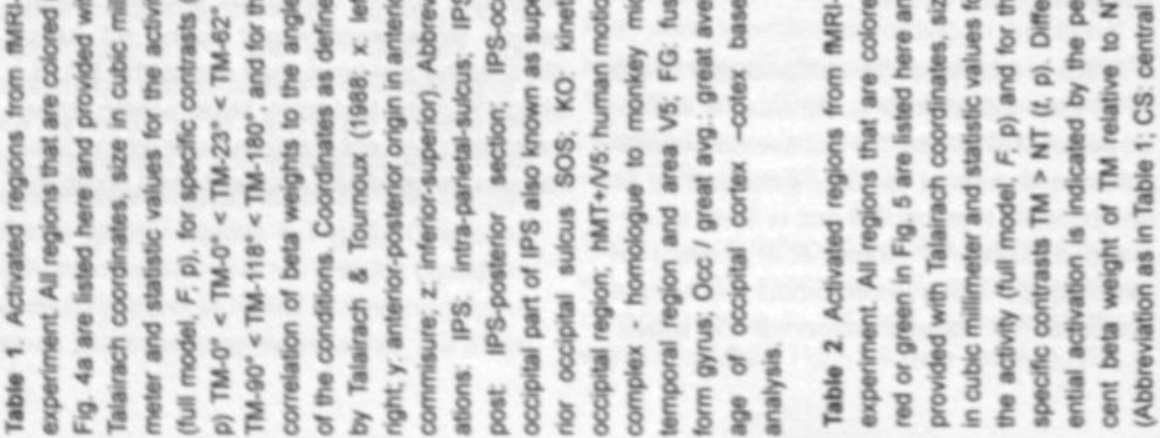

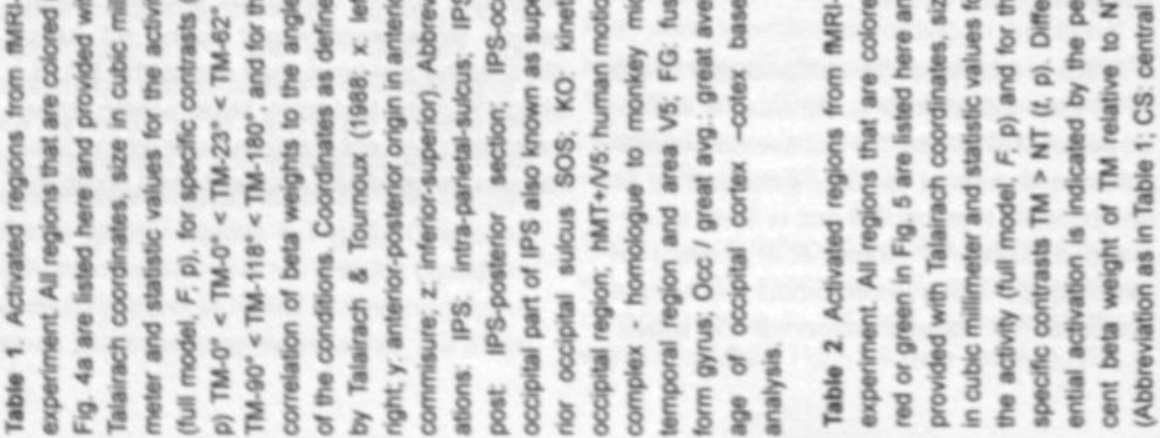




\section{Discussion}

\section{Psychophysics}

Psychophysical experiments revealed that transparent motion is perceived if dot distance exceeds $50 \%$ of the motion stretch. This scale-independent result is surprising because one would expect a constant distance value, if transparent motion perception would be a simple consequence of receptive field properties, i. e. averaging of opponent motion vectors at small distances and preserving motion preference at larger distances. Instead of a constant distance value we observed a fix ratio for the tested distances between $0.7^{\prime}$ and $14^{\prime}$. This leads to the quite remarkable conclusion that with increasing motion stretches, more distance is required to render motion transparency.

Qian et al. (1994b) proposed a model that incorporates spatial pooling and motion opponency mechanism and seeks to account for the fact that some stimuli consisting of two moving components elicit the perception of motion transparency (like the TM stimulus) while others (like the NT stimulus) do not. In this model, suppressive interactions occur within many small subunits each of which receives inhibitory inputs from detectors tuned to different directions of motion. This suppressive interaction occurs in spatially restricted subunits and prevents TM percepfion in conditions in which opponent moving dots are in close vicinity and supports TM perception if the distance between opponent moving dots is large.

The results of our psychophysical experiments are difficult to explain by spatially restricted suppression alone. Thus, the length of the motion streaks needs to be considered as an additional variable, requiring extension of the model proposed by Qian. A refined model should incorporate that moving features induce smeared representations owing to the temporal integration of the visual system resulting in the perception of oriented contours. Their orientation can serve as an additional cue to recognize the direction of motion (Geisler, 1999). This combination of complementary motion cues might explain that the ratio between the streak length and streak distance $(>50 \%)$ rather than distance alone was relevant for motion perception in our experiments. The combination of the two motion cues could take place at stages in the visual processing stream where both cues are available or it could involve a distributed net of specialized regions.

\section{Human motion complex}

The fMRI results suggest that hMT+N5 is the most important cortical area along the dorsal stream for the integration of local motion cues. Areas of the dorsal stream were more active if the local motion cues were perceptually integrated into coherently moving surfaces (fMRI-b). In addition we found that activation was stronger, when two transparent surfaces rather than one single surface were perceived (fMRI-a). Activity increased with the angle between the two motion trajec. tories. As suggested by animal experiments (Treue et al., 2000), this increasing activity could be related to the recruitement of two neuronal populations that largely overlap for small angles (lower activity) and exhibit less overlap for large angles (high activity).

\section{Decreasing activity for increasing}

Interestingly, parietal-IPS showed the reverse response profile as compared to hMT+N5 with decreasing activity when the angle between the two motion trajectories increased. This inverse trend might reflect a correlation with the tendency to perform spatial attention shifts. A coherently moving surface (small angles) leads to a single spatial attention shift tendency while two transpar- 
ently moving surfaces (large angles) reduce this tendency due to mutually cancelling shift directions. This interpretation is consistent with the functional role of putative human LIP (Sereno et al., 2001), which responds with increased activity for remembered target positions.

\section{Other visual areas}

Early visual areas showed little differential activation. Higher visual areas of the dorsal $(\mathrm{hMT}+$ N5, V7) and ventral visual stream (FG) showed more differential activation. These areas correspond well to the network of areas that are involved in the reconstruction of 3D-shapes from motion (Goebel et al. 2000).

KO has been reported to respond specifically to motion contours (Orban et al., 1995: Van Oostende et al., 1997; Dupont et al., 1997). Motion contours were produced by the target regions in MMRI-b but they were only perceived in TM stimuli. Activation in presumed $\mathrm{KO}$, however, was not markedly increased by TM stimuli indicating either that motion contours were not extracted from transparent displays at the level of KO. or our procedure was not sensitive enough for the detection of such effects. The same holds true for V1, which has also been reported to respond to motion contours (Reppas et al., 1997), but whose activation was not markedly increased by TM stimuli. This agrees with the notion that differently moving dots first need to be integrated into different surfaces at the level of $\mathrm{hMT}+1$ V5, before contours can be computed.

Increased activation in the FEF around the somatosensory and motor cortex was seen in fMRI-b during conditions of TM and is most likely related to the task and the response, as it is not seen in the fMRI-a experiment when no responses were required. In agreement with this hypothesis, this frontal activation was more pronounced in the hemisphere contralateral to the response hand. The reason for the differential enhancement of this motor related activity is that response frequency was $60 \%$ higher in the TM than in the NT condition.

\section{Conclusion}

Human MT+N5 responded to subtle changes of parameters relevant for the perception of transparent motion and is therefore identified as an important motion integration site in the human brain. This finding is in perfect agreement with single cell recordings from monkey MT (Qian and Andersen, 1994; Heeger et al., 1999) and previous fMRI results (Heeger et al., 1999). In addition psychophysical testing revealed new spatio-temporal interactions relevant for transparent motion perception and furthermore a network of areas comprising of region IPS-occ (presumed V7), and a region in the FG that responded also well to transparent motion and seemed to be tightly linked to the response pattern of hMT+N5. In contrast parital-IPS (presumed human LIP) responded stronger for coherent motion and less for transparent motion indicating a posible involvement in coding coherent spatial cues. These new findings are compatible with the idea that presumed $V 7$ and $F G$ benefit from or participate in the integration process accomplished at the level of hMT +N5. 


\section{References}

Adelson,E.H. and Movshon, J.A. 1982. Phenome-
nal coherence of moving visual patterns. Nature 300: 523-525.

Andersen,G.J. 1989 . Perception of three-dimensional structure from optic flow without locally smooth velocity. J Exp Psychol Hum Percept Per. form 15:363-371

Boynton, GM. Engel,S.A., Glover, G. H. and Heeger,D.J. 1996 . Linear systems analysis of functional magnetic resonance imaging in human $V_{1}$ J Neurosci 16:4207-4221.

Dierks, T., Linden,D.E.J., Jandl,M., Formisano,E. Goebel, R., Lanfermann, H. and Singer,W 1999 Activation of Heschl's gyrus during auditory hallucinations. Neuron 22:615-621

Dupont, P. De Bruyn, B., Vandenberghe, R. Rosier.A.M. Michiels,J., Marchal,G. Mortelmans, L. and Orban,G.A. 1997. The kinetic occipital region in human visual cortex. Cereb Cortex 7:283-292.

Engel,S.A., Rumelhart,D.E., Wandell,B.A., Lee, A.T. Glover,G.H., Chichilnisky.E.-J., and Shadlen,M.N. 1994. AMRI of human visual cortex. Nature 369.525

Geisler,W. 1999 Motion streaks provide a spatial code for motion direction. Nature 400 65-69.

Gibson,E.J., Gibson,J.J., Smith, O.W. and Flock, $H$. Motion parallax as a determinant of perceived depth. Journal of Experimental. Psychology 58:40-51, 1959 (Abstract)

Goebel,R., Khorram-Sefat,D., Muckli,L. Hacker. H. and Singer,W 1998. The constructive nature of vision: direct evidence from functional magnetic resonance imaging studies of apparent motion and motion imagery. Eur J Neurosci 10:1563. 1573

Goebel, R., Naumer, M., Muckli, L., van Meer, H. Engel, A. K. Zanella, F. E., and Singer, W Struc ture-from-motion of three dimensional objects differentially activates specialized areas in the ventral processing stream. Soc. Neurosci.Abstr. 26[2], 1584.2000

Hadjikhani,N. Liu.A.K, Dale.A.M., Cavanagh,P. and Tootell,R.B.H. 1998. Retinotopy and color sensitivity in human visual cortical area V8. Nat Neurosci 1:235-241.

Heeger,D.J., Boynton,GM., Demb,J.B., Seidemann,E, and Newsome,WT. 1999 Motion opponency in visual cortex. J Neurosci 19:7162. 7174

Kolb,F.C. and Braun,J. 1995. Blindsight in normal observers. Nature 377:336-338

Krauskopf,J. and Farell,B. 1990. Influence of colour on the perception of coherent motion Nature 348:328-331

Kriegeskorte, N. and Goebel,R. 2001. An efficient algorithm for topologically correct segmentation of the cortical sheet in anatomical MR volumes Neurolmage 14:329-346

Linden,D.E.J. Kallenbach U. Heinecke.A. Singer,W. and Goebel,R 1999 The myth of upright vision. A psychphysical and functional imaging study of adaptation to inverting spectacles. Perception 28.469-481

Lueck,C.J., Zeki,S. Friston,K.J., Deiber,M.P. Cope,P. Cunningham,V.J., Lammertsma A.A. Kennard,C., and Frackowiak, R. S 1989. The colour centre in the cerebral cortex of man Nature 340:386-389

Mulligan,J.B 1992 . Anisotropy in an ambiguous kinetic depth effect. J Opt Soc Am [A] 9:521-529

Orban,GA., Lagae,L., Raiguel,S., Xiao,D , and Maes, H. 1995. The speed tuning of medial superior temporal (MST) cell responses to optic-fiow components. Perception. 24.269-285

Petit,L. and Haxby.J.V 1999. Functional Anatomy of Pursuit Eye Movernents in Humans as Revealed by AMRI. J Neurophysiol 82:463-471.

Qian, N. 1997. Binocular disparity and the perception of depth. Neuron 18:359-368

Qian, N and Andersen,RA. 1994. Transparent motion perception as detection of unbalanced motion signals. II. Physiology I Neurosci 14:7367.7380

Qian, N. Andersen, R.A. and Adelson, E. H. 1994a Transparent motion perception as detection of unbalanced motion signals I. Psychophysics If Neurosci 14.7357.7366 
Qian, N. Andersen, R.A., and Adelson, E.H. 1994b. Transparent motion perception as detection of unbalanced motion signals. III. Modeling. J Neurosci 14.7381 .7392

Reppas,J B., Niyogi,S, Dale, A.M., Sereno,M.I. and Tootell,R.B. 1997 Representation of motion boundaries in retinotopic human visual cortical areas. Nature 388:175-179.

Sereno, M I., Dale, A.M., Reppas, J B., Kwong. K. K. Belliveau.J. W. Brady, T.J., Rosen,B.R., and Tootell, R. B. 1995. Borders of multiple visual areas in humans revealed by functional magnetic resonance imaging. Science 268 889-893

Sereno MI, Pitzalis S. Martinez A (2001) Mapping of contralateral space in retinotopic coordinates by a parietal cortical area in humans. Science 294: 1350-1354

Snowden,R.J.. Treue,S. Erickson, RG, and Andersen,RA. 1991. The response of area MT and $V 1$ neurons to transparent motion. J Neurosci 11:2768-2785.

Sunaert, S. Van Hecke,P. Marchal,G. and Orban,GA 1999. Motion-responsive regions of the human brain. Exp Brain Res 127:355-370.

Talairach,J. and Tournaux,P. Co-planar Stereotaxic Atlas of the Human Brain. New York, NY: Thieme, 1988.

Tootell, R B. Mendola, J.D. Hadjikhani, N.K. Liu.A.K. and Dale.A.M. 1998a. The representation of the ipsilateral visual field in human cerebral cortex. Proc Natl Acad Sci U S A 95:818-824.

Tootell,R.B.H. Hadjikhani,N.K, Mendola,J.D. Marrett.S. and Dale.A.M. 1998b. From retinotopy to recognition: MRI in human visual cortex Trends Cognit Sci 2:174-183.

Van Oostende,S., Sunaert, S. Van Hecke,P., Marchal, G. and Orban, GA. 1997 . The Kinetic Occipital (KO) Region in Man: An MRI Study Cereb Cortex 690-701

Wallach, H. and O'Connell,D.N. 1953. The kinetic depth effect Journal of Experimental Psychology 45: 205-217.

Zeki.S. McKeefry, D.J., Bartels, A. and Frackowlak, R.S. 1998. Has a new color area been discovered? Nat Neurosci 1.335-336.

\section{Acknowledgments}

The study was supported by the Max Planck Society and the Consortium for the Investigation of Consciousness. We thank David E. J. Linden and Nikolaus Kriegeskorte for valuable comments on the manuscript and Renate Ruhl for help with the figures. 


\section{Chapter 5}

\section{The constructive nature of vision: Direct evidence from fMRI studies of apparent motion and motion imagery}

This chapter corresponds to:

Rainer Goebel ${ }^{1}$, Darius Khorram-Sefat ${ }^{2}$, Lars Muckli ${ }^{1}$, Hans Hacker ${ }^{2}$ and Wolf Singer ${ }^{1}$ (1998) The constructive nature of vision: Direct evidence from fMRI studies of apparent motion and motion imagery. European Journal of Neuroscience, 10, 1563-1573.

${ }^{1}$ Max-Planck-Institut fur Hirnforschung. Frankfurt a. M., Germany ${ }^{2}$ Klinikum der Johann Wolfgang Goethe-Universitat, Abteilung Neuroradiologie. Frankfurt a. M., Germany 


\section{Abstract}

Echoplanar functional magnetic resonance imaging was used to monitor activation changes of brain areas while subjects viewed apparent motion stimuli and while they were engaged in motion imagery. Human cortical areas MT (V5) and MST were the first areas of the "dorsal" processing stream which responded with a clear increase in signal intensity to apparent motion stimuli as compared to flickering control conditions. Apparent motion of figures defined by illusory contours evoked greater activation in V2 and MT/MST than appropriate control conditions. Several areas of the dorsal pathway (V3A, MT/MST, areas in the inferior and superior parietal lobule) as well as prefrontal areas including FEF and BA 9/46 responded strongly when subjects merely imagined moving stimuli which they had seen several seconds before. The activation during motion imagery increased with the synaptic distance of an area from $\mathrm{V} 1$ along the dorsal processing stream. Area MTMST was selectively activated during motion imagery but not during a static imagery control condition. The comparison between the results obtained with objective motion, apparent motion and imagined motion provides further insights into a complex cortical network of motion-sensitive areas driven by bottom-up and top-down neural processes. 


\section{Introduction}

Perceptual illusions and visual imagery are important paradigms for the experimental study of constructive aspects of vision and the generation of visual percepts that goes beyond the information contained in the mere physical composition of the stimuli (Zeki et al., 1993). Certain types of illusions arise when the physical properties of a stimulus are supplemented by perceptual features that rely on the assumptions of the brain about what it expects in the outside world. For example, when stimuli separated in space are turned on and off in alternation at appropriate temporal intervals (Kolers, 1972), subjects perceive one stimulus moving between the two stimulus positions (apparent motion) rather than two stationary flickering stimuli. Visual imagery, on the other hand, may be performed in the complete absence of visual stimuli. Thus, when a subject is requested to imagine a previously seen visual scene, the task usually consists in the construction of a visual image purely from stored information. In order to study constructive aspects of motion perception we reduced gradually the amount of objective motion perception and increased the amount of internally generated motion representations in a series of experiments using objectively moving stimuli, apparent motion stimuli, stimuli inducing apparent motion of figures defined by illusory contours and motion imagery conditions. Part of this work has previously appeared in abstract form (Goebel et al., 1996, Goebel et al., 1997).

Previous brain imaging studies using either positron emission tomography (PET) or functional magnetic resonance imaging (fMRI) have shown that human cortical areas MT (V5) and MST respond with increased activity to moving stimuli (Watson et al., 1993; Tootell et al., 1995b; McCarthy et al., 1995) and stationary stimuli inducing illusory motion (Zeki et al., 1993; Tootell et al., 1995a). We investigated to what extent apparent motion stimuli also activate the motion-selective areas MT and MST. Since it is known that these areas respond to some degree to flickering stimuli (Tootell et al., 1995b), appropriate control conditions had to be specified which differ from the apparent motion stimuli only in the relative timing of appearing objects. Additionally, form-motion interactions were investigated by inducing apparent motion of squares whose outlines were defined by illusory contours. This paradigm integrates two perceptual illusions and should provide additional insight into the interaction of brain regions responsible for extracting different stimulus properties.

We further investigated whether the areas activated by apparent motion stimuli are also activated when moving stimuli are solely imagined. Previous brain imaging studies have shown that mental imagery activates some of the same brain regions as in visual perception (e.g., Kosslyn et al., 1995). It is. however, unclear whether small circumscribed extrastriate visual areas responsible for the analysis of specific stimulus properties can be selectively activated by imagery. We examined this issue for the motion-selective areas MT and MST by comparing their response patterns during imagery of moving stimuli with imagery of static stimuli.

\section{Materials and methods}

\section{Subjects}

Ten subjects without a neurological or psychiatric history participated in this study. Age ranged from 25 to 32 years, and there were six males. Informed consent was obtained from each subject. 


\section{Experimental protocol}

Visual stimuli were delivered under computer control (Digital DECpc Celebris XL 590) to an LCD display panel (Sharp QA-1000) and a high-luminance overhead projector (Elmo HP-285P) in experiments $1-3$ and to a highluminance LCD projector (EIKI LC-6000) in experiment 4. Subjects were in supine position and viewed the screen through an adjustable mirror fixed to the head coil. The image was back-projected onto a frosted screen positioned at the foot end of the scanner. Visual stimuli were generated in real time using the ELSA Winner $2000 \mathrm{Pro} / \mathrm{X}$ graphics adapter and the ELSA Powerlib C library.

\section{Activation tasks}

Each experiment consisted of 104-128 measurements, each sampling 10-15 oblique transversal slices running either approximately in parallel to the calcarine fissure (experiments 1 - 3) or approximately in parallel to the AC-PC line (experiments 3 and 4 ). Each experimental condition lastet $24 \mathrm{~s}$. This allowed the acquisition of eight measurements per condition. In experiment 4, the initial and final fixation condition consisted of only four measurements. In all conditions a small fixation cross appeared in the center of the screen. The fixation cross was the only stimulus present during fixation conditions and imagery conditions. Each experiment included an objective motion stimulus consisting of $\mathbf{4 0 0}$ white dots moving radially outward on a black background (visual field: $30 x$ wide by $23 \times$ high, dot size: $0.06 \times \times 0.06 \times$, dot velocity: $3.6 \times / \mathrm{s}-14.4 \times / \mathrm{s}$ ). This stimulus is known to produce a clear MT/MST response without provoking eye movements (Tootell et al, 1995b). Additionally, a static stimulus which consisted of 400 stationary dots was used in each experiment.

\section{Apparent motion I}

In the first experiment, perception of apparent motion was induced with two concentric rings (diameters: $3 x$ and 10x) that appeared in alternation, separated by a short blank interval of $32 \mathrm{~ms}$ (Fig. 2A). We opted for this stimulus which was perceived as a single shrinking and expanding ring by all subjects $(n=10)$ because it does not elicit eye movements. As a control stimulus devoid of apparent motion cues both concentric rings were turned on and off simultaneously. Thus, this flickering control condition differed from the apparent motion condition only in the relative timing between the large and the small ring. Two flickering control conditions were used (flickering control I and II, see Fig. 2A). The sequence of stimulation conditions (see Fig. 2B) consisted of: Fixation, Objective motion. Fixation, Stationary dots, Fixation, Apparent motion, Fixation, Flickering control. This sequence was repeated twice using flickering control I in the first repetition and flickering control II in the second repetition. Additionally, a final fixation condition was included resulting in 13 stimulation conditions (104 measuerements).

\section{Apparent motion II}

In the second experiment the stimuli used to generate the perception of apparent motion were squares whose outlines consisted of subjective contours (Kanizsa, 1979). These illusory squares were presented in alternation on the left and right side of the fixation spot (Fig. 3A). In order to generate the percept of an appearing and disappearing square the four "pacmen" defining a square were opened and closed simultaneously so that observers $(n=7)$ saw either a Kanizsa square or four filled circles (diameter: 0.9x) at the respective location. Kanizsa squares were centered at an eccentricity of $4.5 x$ and were $2.2 x$ in length. In the control condition without motion cues the illusory Kanizsa squares appeared 
and disappeared simultaneously on both sides of the fixation cross whereas in the apparent motion condition they appeared and disappeared in alternation (Fig. 3A). Despite of this change in relative timing across hemifields, stimulation conditions within each hemifield remained the same in both conditions. In the control condition subjects reported that they were seeing two squares appearing and disappearing in alternation while in the apparent motion condition they reported that they were seeing a single square moving between the two stimulus locations. In two additional no-contour control conditions the pacmen were opened outward and thus did not produce illusory squares (Fig. 3A). A sequence of 13 stimulation conditions was used consisting of Fixation, Objective motion, Fixation, Static, Fixation, Subjective-contour apparent motion, Fixation, Subjective-contour flickering control, Fixation, No-contour apparent motion, Fixation, Nocontour flickering control, Fixation.

\section{Motion imagery I}

In the third experiment we examined whether motion-sensitive areas are activated when subjects solely imagine moving stimuli. After subjects $(n=5)$ had seen one of three motion stimuli for $24 \mathrm{sec}$ (radially moving dots. apparent motion of subjective contours or rotating grating), they were required to fixate a cross for 24 seconds. This long interval was introduced in order for the hemodynamic response to return to baseline level before the start of the following imagery condition. After this fixation period, the fixation cross was turned off for $200 \mathrm{~ms}$, which signalled to the subject that they should now try to imagine the previous motion stimulus as intensely as possible while fixating the cross. After 24 seconds, the cross was turned off again for $200 \mathrm{~ms}$. This signalled to the subject the end of the imagery condition. Motion stimuli were the same as described above except the rotating grating (diameter: $16.7 \times$, frequency:
15) which rotated with $170 \times / \mathrm{sec}$. The experiment was repeated twice. Activity was measured in twelve slices approximately parallel to the calcarine fissure and in 15 axial slices with a transversal-to-coronal angle of 5-10 degrees with respect to the AC-PC line. The sequence of 15 stimulation conditions consisted of Fixation, Static, Fixation, Motion I (radially moving dots), Fixation, Motion imagery I, Fixation, Motion II (subjective-contour apparent motion stimulus of experiment II). Fixation, Motion imagery II, Fixation, Motion III (rotating grating), Fixation, Motion imagery III, Fixation. The sequence of stimulation conditions (triplets of motion-fixation-imagery) was randomized across subjects.

\section{Motion imagery II}

In the fourth experiment, imagery of moving stimuli was compared with imagery of static stimuli. The experimental procedure was the same as in the previous experiment, except that either a static or a moving stimulus was shown prior to the respective imagery period. One static stimulus consisted of the standard static control condition (400 randomly positioned dots). A stationary frame of the subjective-contour apparent motion stimulus of experiment 2 was used as the second static stimulus. The dynamic versions of these stimuli (radially outward moving dots, subjective-contour apparent motion stimulus) were used in the two motion conditions. Activity was measured in 15 slices approximately parallel to the calcarine fissure and in 15 axial slices with a transversal-to-coronal angle of 1-5 degrees with respect to the AC-PC line. The sequence of 17 stimulation conditions consisted of Fixation, Static I (stationary dots), Fixation, Static imagery (SI) I, Fixation, Motion I (radially moving dots). Fixation. Motion imagery (MI) I, Fixation, Static II (stationary frame of subjective-contour apparent motion stimulus of experiment 2). Fixation, Static imagery II, Fixation, Motion II (Subjective-contour apparent motion of experiment 
2), Fixation, Motion imagery III, Fixation. In the first and last fixation condition only four measurements were acquired resulting in $128\left(15^{*} 8+2^{*} 4\right)$ measurements.

\section{Eye movements}

In order to verify whether subjects were able to fixate the cross during apparent motion and motion imagery conditions, the third experiment was performed outside the scanner monitoring eye movements with horizontal and vertical electro-oculograms.

\section{MRI acquisition}

Echo-planar images were collected on a 1.5T scanner (Siemens Magnetom Vision) using the standard head coil and a gradient echo echoplanar sequence $(T R=3000 \mathrm{~ms}$. TE $=$ $66 \mathrm{~ms}$, flip angle $=900, F O V=210 \mathrm{~mm} \times 210$ $\mathrm{mm}$, slice thickness $=3 \mathrm{~mm}$, imaging matrix $=$ $64 \times 64$, voxel size $=3.2 \times 3.2 \times 3 \mathrm{~mm}$ ). In experiments 2 - 4 the Siemens Magnetom gradient overdrive was used allowing functional scans with high spatial resolution (TR = $3000 \mathrm{~ms}, \mathrm{TE}=69 \mathrm{~ms}$, flip angle $=900, F O V$ $=210 \mathrm{~mm} \times 210 \mathrm{~mm}$, slice thickness $=3 \mathrm{~mm}$, imaging matrix $=128 \times 128$, voxel size $=1.6 \times$ $1.6 \times 3 \mathrm{~mm}$ ). Before each set of functional scans in experiments 1 and 2 , we recorded a T1-weighted series of images with the same orientation, slice thickness, and field of view as the functional scans. In experiments 3 and 4, a T1-weighted 3D MP RAGE scan lasting 8 minutes was recorded in the same session as the functional measurements. Additional T1-weighted 30 data sets tuned to optimize the contrast between gray versus white matter were recorded for each subject in separate recording sessions lasting 24 minutes.

\section{Data analysis}

Data analysis, including preprocessing (motion correction, Gaussian spatial (FWHM $=3$ pixel) and temporal (FWHM=2 measurements) smoothing. linear trend removal), correlation analysis, determination of Talairach coordinates, volume rendering. surface rendering and cortex flattening was performed using custom software (BrainVoyager, Goebel, 1996, 1997) on a DEC Alpha $500 \mathrm{MHZ}$ Personal Workstation with the Windows NT 4.0 operating system. Statistical mapping. motion correction and Talairach transformation was crossvalidated for three subjects using AFNI 96 (Cox, 1996) on a Pentium $166 \mathrm{MHZ}$ Gateway 2000 PC running the Linux 2.0 operating system. Prior to statistical analysis the time series of functional images was aligned for each slice in order to minimize the effects of head movement. For each slice the third recorded functional image was used as a reference image to which all other images of the slice time series were registered. In order to evaluate statistically the differences between experimental conditions cross-correlation analysis was applied. For the computation of correlation maps, the stimulation protocol served as the basis of appropriate reference functions reflecting experimental and control conditions (experimental condition $=1$, control condition $=0$ ) On a pixel-by-pixel basis the signal time course was cross-correlated with a reference function (Bandettini et al, 1993). Pixels were included into the statistical map if the obtained correlation value was greater than 0.4. given lag values of 1 and 2 (corresponding to a 3-9 secs delay after the beginning of a stimulation condition in order to adapt to the hemodynamic response). Cross-correlation maps were superimposed both on the original functional scans as well as onto $\mathrm{T} 1$-weighted $2 \mathrm{D}$ or $3 \mathrm{D}$ anatomical reference scans.

The data for statistical comparisons consisted of the mean time course of all voxels of an analyzed area. Based on this data the mean of the raw fMRI signal for each subject and condition in a given experiment was computed. These mean values were analyzed 
using ANOVA and post-hoc pairwise comparisons using stimulus condition as a withingroup factor. The obtained p-values were corrected for multiple comparisons. Values of percent signal change averaged across subjects were computed on the basis of the difference between the mean values of the fMRI signal in each experimental condition and the mean fMRI signal in the fixation periods for each individual subject.

Statistical maps were transformed into the high-resolution 3D data sets and interpolated to the same resolution (voxel size: $1.0 \times 1.0 \times$ $1.0 \mathrm{~mm}$ ). For each subject the structural and functional 3D data sets were transformed into Talairach space (Talairach and Tournaux, 1988) which allowed us to compare activated brain regions across different experiments and across different subjects and to determine Talairach coordinates of these regions. Talairach transformation was performed in two steps. The first step consisted in rotating the $3 \mathrm{D}$ data set of each subject to be aligned with the stereotaxic axes. For this step the location of the anterior commissure (AC) and the posterior commissure (PC) as well as two rotation parameters for midsagittal alignment had to be specified manually. In the second step the extreme points of the cerebrum were specified. These points together with the AC and PC coordinates were then used to scale the $3 D$ data sets into the dimensions of the standard brain of the Talairach and Tournaux atlas using a piecewise affine and continuous transformation for each of the 12 defined subvolumes.

Striate and extrastriate cortical areas V1, V2. V3, VP, V3A and V4V were discriminated based on anatomical location and functional properties (Sereno et al., 1995; Tootell et al. 1996; Van Essen and Drury. 1997). The delineation of these areas was validated for each subject using results from separate recordings for generating retinotopic maps (compare Engel et al., 1994: DeYoe et al., 1994: Sereno et al., 1995), sampling 12 contiguous slices approximately in parallel to the calcarine fissure $(T R=3000 \mathrm{~ms}$, $T E=69 \mathrm{~ms}$, flip angle $=900, F O V=200 \mathrm{~mm} \times 200 \mathrm{~mm}$, slice thickness $=3 \mathrm{~mm}$, gap thickness $=0$ $\mathrm{mm}$, imaging matrix $=128 \times 128$, voxel size $=$ $1.6 \times 1.6 \times 3 \mathrm{~mm})$. Within the same recording session, an eccentricity mapping and a polar angle mapping experiment were performed. In the eccentricity mapping experiment, white and black checks flickering at $4 \mathrm{~Hz}$ formed into a ring-shaped configuration. The ring started with a radius of 10 and slowly expanded to a radius of 120 within 96 seconds. In the polar angle mapping experiment, flickering white and black checks formed into a ray-shaped configuration subtending 22.50 in polar angle. The ray started at the left horizontal meridian and slowly rotated clockwise for a full cycle of 3600 within 96 sec. Each mapping experiment consisted of four repetitions of a full expansion or rotation, respectively. Retinotopy of eccentricity and polar angle was revealed with cross-correlation analysis selecting the lag value resulting the highest correlation value for a particular voxel. The obtained lag value finally determined the pseudocolour for that voxel as well as for corresponding polygons on reconstructed surfaces (Fig. 1B, C). The recorded high resolution $\mathrm{T} 1$-weighted $3 \mathrm{D}$ recordings were used for surface reconstruction (Fig. 1A) of the posterior part of subjects' brains including mainly the occipital lobe. The white matter within this region was segmented with a region-growing method. The surface reconstruction procedure started with a sphere (recursively tesselated icosahedron) which slowly wrapped around the segmented white matter. In order to avoid topological defects and to let the surface smoothly grow into deep sulci, we defined a dynamic mesh algorithm which automatically invents new polygons at places where the length of triangle edges is more than 1.2 times the average 

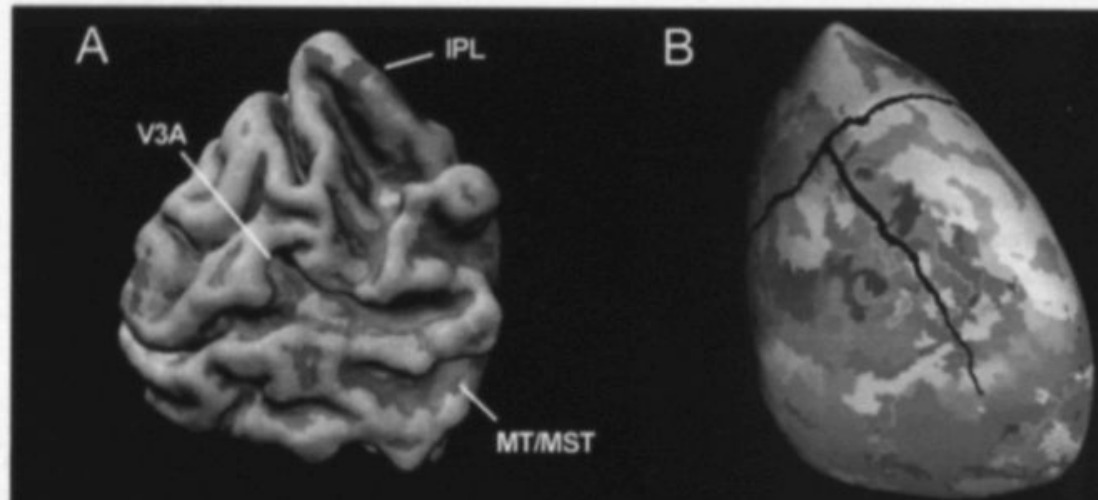

C

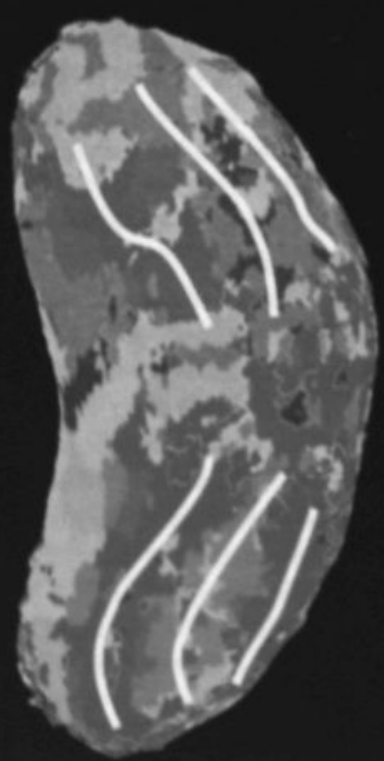

D

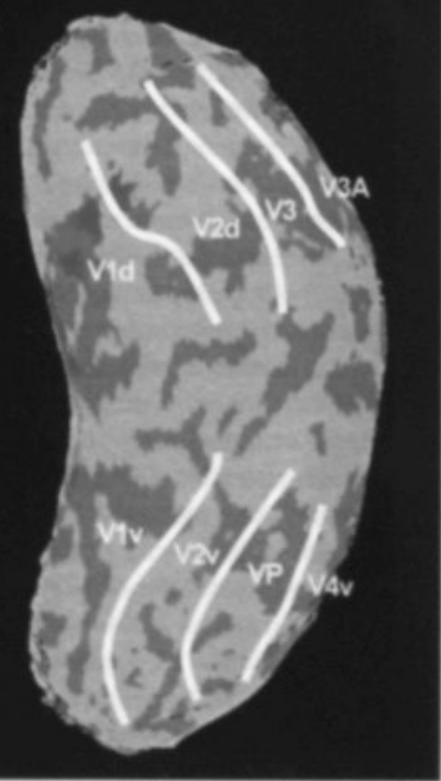

Figure 1. Results of boundary determination of retinotopic cortical areas V1, V2, V3, VP. V3A and V4v of one subject (A) Lateral view of the reconstructed surface of the posterior portion of the left hemisphere of the subject's brain. The statistical map comparing the objective motion condition with the static condition is projected to the reconstructed surface revealing motion-selective areas including V3A. MTMST as well as a region in the inferior parietal lobule (IPL). (B) Ventro-medial view of the same surface after cortex "inflation". The retinotopic eccentricily map is projected onto the reconstructed surface. Retinotopic responses are represented by using a pseudocolour scale. Responses ranging from central to peripheral stimulation are coded red through yellow through green through blue. Black lines show cuts $2.5 \mathrm{~cm}$ anterior to the occipital pole and roughly along the fundus of the calcarine sulcus. (C) Flattened visual cortex of the posterior part of the hemisphere. The polar angle map is projected onto the flattened surface representation. Responses to right upper visual field are coloured yellow (horizontal meridian) to red (vertical mendian), responses to right lower visual field are coloured blue (horkzontal meridian) to green (vertical meridian). Area boundaries (while lines) are drawn at vertical and horizontal meridian representations. (D) The same statistical map as in (A) comparing objective motion condition and static condition projected to the cut and flattened cortex showing activity in areas V1, V2d and V3A. 
A
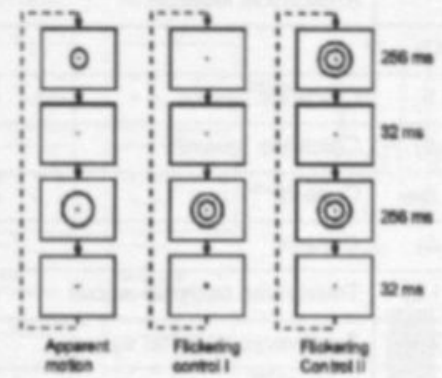

B
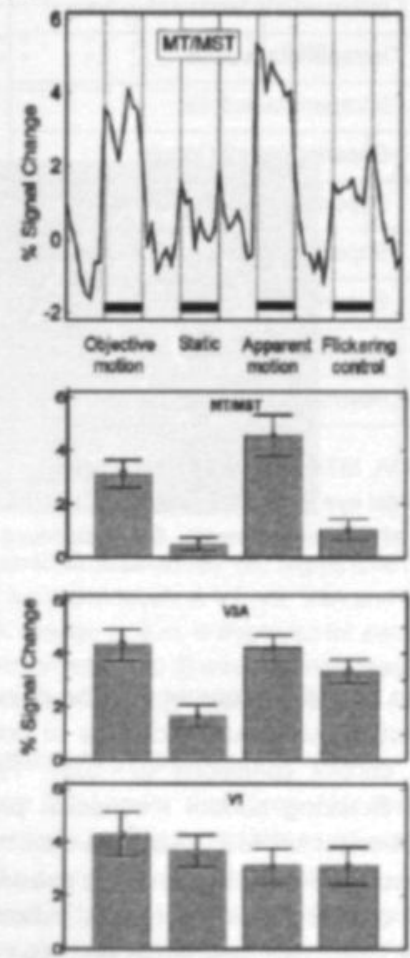

edge length. Within a typical surface reconstruction run, the mesh grows from initially 20480 triangles to $60000-80000$ triangles. The reconstructed white matter surface is finally expanded into the gray matter. In order
Figure 2. Main experimental conditions and results of experiment 1. The sequence of stimulation conditions consisted of: Fixation, Objective motion, Fixation, Static, Fixation, Apparent motion, Fixation, Flickering control. This sequence was repeated twice. (A) The apparent motion stimulus consisted of two concentric rings which appeared in alternation, separated by a short blank interval to enhance the perception of apparent motion. In the first repetition of the sequence the flickering control I and in the second repetition the fickering control II condition was used. (B) Signal time course in cortical area MT/MST (top) and statistical comparison of activation leveis in V1, V3A and MTMST (bottom) averaged over 10 subjects. Mean percent signal change \pm s. $\mathrm{e} . \mathrm{m}$. is displayed for each condition.

to avoid cross-talk during sampling of functional data between both banks of a sulcus, the final surface (Fig. 1A) is, however, kept closer to the white matter border (below estimated position of layer 4). Prior to surface flattening. the cortical surface was inflated and cut along the calcarine fissure and along a coronal slice $-3 \mathrm{~cm}$ anterior to the occipital pole (Fig. 1B). The surface was then slowly unfolded against a coronal plane at the occipital pole minimizing areal distortions. Based on the polar angle mapping experiment which includes stimulation of the horizontal and vertical meridian, the boundaries of retinotopic cortical areas V1, V2, V3, VP, V3A and V4V were defined on the cortical surface for each subject (Fig. 1C, D).

In order to delineate MTMST as well as other motion-selective areas we defined regions that were significantly more strongely activated during the objective motion condition than during the static condition as regions of interest (Tootell et al., 1995b). In experiments 3 and 4, additional regions of interest (ROIs) were defined a posteriori by comparing all imagery conditions with the fixation conditions. 


\begin{tabular}{|l|l|l|r|r|l|}
\hline \multirow{2}{*}{ Area } & \multicolumn{4}{|c|}{ Talairach coordinates } & Anatomical location \\
\hline & & $x(S D)$ & $y(S D)$ & $z(S D)$ & \\
\hline V1 & L & $-7.4(3.5)$ & $-87.6(2.1)$ & $-7.4(3.5)$ & Calcarine fissure \\
\hline & R & $5.4(1.1)$ & $-84.6(3.8)$ & $-5.8(1.6)$ & Calcarine fissure \\
\hline V2d & L & $-10.8(6.5)$ & $-94.4(5.7)$ & $2.8(3.8)$ & Cuneus \\
\hline & R & $13.2(7.2)$ & $-94.0(6.0)$ & $2.0(4.6)$ & Cuneus \\
\hline V3A & L & $-18.0(6.5)$ & $-91.3(4.6)$ & $13.3(1.2)$ & Transverse occipital sulcus \\
\hline MTMST & L & $10.8(6.8)$ & $-90.8(3.5)$ & $13.6(2.3)$ & Transverse occipital sulcus \\
\hline & R & $-45.2(3.1)$ & $-56.8(2.8)$ & $4.6(3.9)$ & Intermediate temporal gyrus \\
\hline IPL & L & $-19.3(6.0)$ & $-76.8(7.1)$ & $36.0(4.8)$ & Intraparietal sulcus \\
\hline & R & $21.2(3.3)$ & $-75.0(4.1)$ & $36.4(2.3)$ & Intraparietal sulcus \\
\hline SPL & L & $-25.8(6.8)$ & $-54.8(3.6)$ & $52.4(4.0)$ & Superior parietal lobule \\
\hline & R & $17.8(7.3)$ & $-54.0(3.4)$ & $52.2(3.4)$ & Superior parietal lobule \\
\hline FEF & L & $-43.8(7.9)$ & $1.4(4.7)$ & $39.6(4.7)$ & Superior part of precentral sulcus \\
\hline & R & $43.6(9.3)$ & $2.6(5.0)$ & $40.8(7.1)$ & Superior part of precentral sulcus \\
\hline BA 9/46 & L & $-34.2(5.2)$ & $31.3(11.4)$ & $23.5(7.9)$ & Intermediate frontal gyrus \\
\hline & R & $38.0(4.5)$ & $26.8(9.3)$ & $29.8(8.5)$ & Intermediate frontal gyrus \\
\hline
\end{tabular}

Table 1. Talairach coordinates of areas V1, dorsal V2 (V2d.) V3A. MTMMST, area in the inferior parietal lobule (IPL), area in the superior parietal lobule (SPL), frontal eye field (FEF) and area in Brodman area $9 / 46$ averaged over five subjects participating in all four experiments. SD = standard deviation $[\mathrm{mm}] \mathrm{L}=$ left hemisphere, $\mathbf{R}=$ right hemisphere

\section{Results}

\section{Apparent motion I}

Area V1 responded with largely similar actvation levels to all stimulation conditions, the lowest activation being in both two ring conditions. In area V3A, the objective motion condition, the apparent motion condition and the flickering control evoked similarty large responses, but in contrast to V1 the stationary stimulus was significantly less effective $(p<0.01$, Fig. 28). Area MT/MST responded strongly to both the apparent and the objective motion stimulus but much less to both fickering control conditions ( $p<0.01$, Fig. 2B). The flickering control I condition produced a stronger MTMST response than the flickering control II condition and was used for statistical comparisons. The results indicate that the MTMST complex is the first area in the dorsal pathway that responds selectively to apparent motion. A response pattern very similar to that of MTMST was found in the intraparietal sulcus. 
A
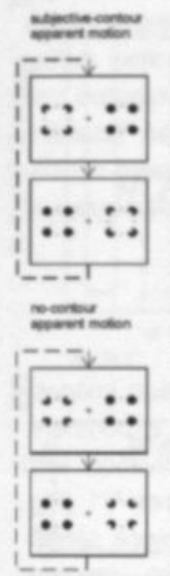

C

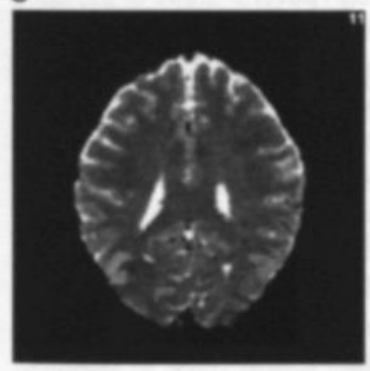

B
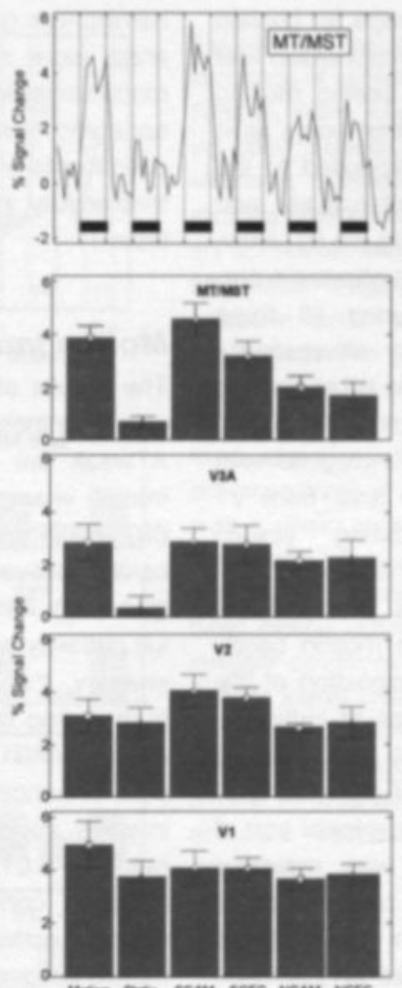

Figure 3. Main experimental conditions and results of experiment 2. The sequence of stimulation conditions consisted of. Fixation, Objective motion, Fixation, Static, Fixation, Subjective-contour apparent motion (SCAM), Fixation, Subjective-contour fickering control (SCFC). Fixation, No-contour apparent motion (NCAM). Fixation, No-contour flickering control (NCFC). (A) in the subjectivecontour conditions four circles or four "pacmen" figures appeared on the left and the right side of the fixation cross. By showing the four "pacmen" figures or the four filled circles observers saw either a Kanizsa square or four disks at the respective location. In the subjective-contour apparent motion condition the Kanizsa squares appeared in alternation on each side. In the subjective-contour fickering control the Kanizsa squares appeared and disap-

peared simultaneously. (B) Signal time course in cortical area MTMST (top) and statistical comparison of activation levels in V1, V2, V3A and MTMST (bottom) averaged over 7 subjects. Mean percent sig. nal change \pm s.e.m. is displayed for each condition. (C) Location of area MTMST of one subject. A correlation map $(>0.3)$ was superimposed on a respective functional scan which was obtained with a EPI sequence with high spatial resolution (voxel size: $1.6 \times 1.6 \times 3 \mathrm{~mm}$ ).

\section{Apparent motion II}

Figure $3 \mathrm{~B}$ shows that area $\mathrm{V} 1$ responded with similar activation levels to all subjectivecontour and no-contour conditions. Area V2 but not $\mathrm{V}_{1}$ and $\mathrm{V} 3 \mathrm{~A}$ responded significantly more strongly to the two conditions with illusory contours than to the respective control conditions with the outward turned pacmen $(p<0.01)$. The stimulus generating apparent motion of figures defined by subjective contours activated MTMST more than the flickering squares $(p<0.01)$ and these in turn were more effective than the outward turned pacmen that did not define Kanizsa squares $(p<0.01)$. Unexpectedly, the difference between the apparent motion and the flickering control condition was smaller than in the first experiment, although the amount of stationary flicker was reduced in the second experiment.

\section{Motion imagery I}

All subjects reported that they were able to evoke mentally a clear motion experience 
during the imagery periods. At the same time they judged their mental image to contain less figural detail than the percept which was generated when they were seeing moving contours. As expected, the same areas as in the previous experiments responded to the initial presentation of the objectively and apparently moving stimuli (Fig. 4A,B). However, most of these areas also showed a surprisingly high activation during all three conditions of motion imagery. Interestingly. the imagery specific activation (ISA) defined as the ratio (motion imagery - fixation) I (objective motion - fixation) increased with the synaptic distance of an area from V1 along the dorsal processing stream (Fig. 4B, Fig. 5A). Area MT/MST was activated approximately half as much in the imagery conditions as in the motion conditions (ISA $=0.6$ ). A close inspection of the time course of signal changes at individual voxels revealed that the ISA was different within subparts of the MT/MST complex. During motion imagery the upper anterior part of MT/MST (presumed MST) was activated almost as strongly as during presentation of objective motion stimuli (ISA $=0.8$ ) while the lower posterior part (presumed MT) showed less activation $(I S A=0.4)$. A weak but signifcant activation with motion imagery was also found in areas V2 and V $3 A$ ( $p<0.01)$, but no significant modulation was detectable in primary visual cortex. In addition to MT/MST several cortical areas in the inferior and superior parietal cortex were strongly and bilaterally activated during motion imagery (Fig. 4B, Fig. 5A). Comparing all imagery conditions with all fixation conditions revealed further regions including the dorsolateral prefrontal cortex, mainly Brodman area (BA) 9/ 46. a region close to the junction of the precentral sulcus and the superior frontal gyrus (BA 6) corresponding according to Paus (1996) to the human frontal eye field (FEF), the anterior cingulate gyrus and the insular gyrus (Fig. 5B). Activation of BA 9/46 was found bilaterally but was higher in the right hemisphere $(p<0.01)$. Most of these higher areas were more strongly activated during motion imagery than during the objective and apparent motion conditions. Mean Talairach coordinates of the areas that showed consistent activity in all subjects are presented in table 1.

\section{Motion imagery II}

The results obtained during motion imagery resemble those of the preceding experiment. A weak but non-significant activation with motion imagery was found in area V1 (Fig. $6 \mathrm{~B})$. All other analyzed areas were signifcantly activated during motion imagery $(p<0.01)$. Although several areas of the dorsal pathway were also activated during static imagery, the activation was much weaker than during motion imagery in most areas. Area MT/MST (Fig. 6A, B) shows no signiffcant enhancement during static and static imagery conditions but a strong enhancement $(p<0.01)$ during motion and motion imagery conditions $(I S A=0.6)$. Additional regions activated during both static and motion imagery (not shown in Fig. 6) included several small areas in the intermediate and inferior frontal gyri, regions in the insular gyrus as well as the anterior cingulate gyrus. The latter responded with roughly equal strength to both types of imagery but showed no response to other stimulus conditions.

\section{Eye movements}

In analyzing the EOG records of the first motion imagery experiment, less than five eye movements of 10 or more per subject during all experimental conditions were detected. 
A
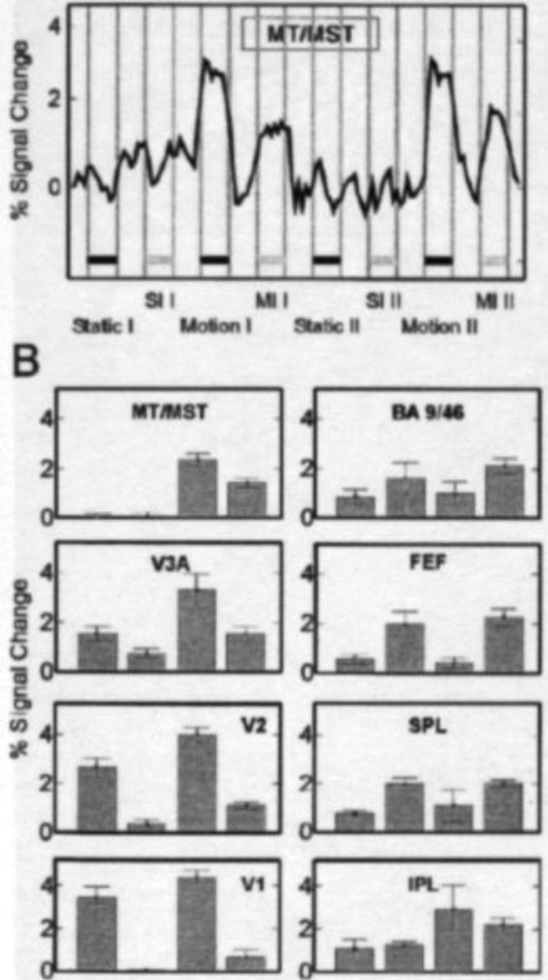

Stafic SI Motion MII
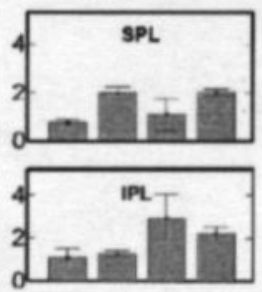

Statc Si Motion Mil

\section{Discussion}

The comparison between the results obtained with objective motion, apparent motion and imagined motion provides new insights into a complex cortical network of motion-sensitive areas driven by bottom-up and top-down neural processes. It has to be conceded that functional MRI provides an indirect measure of neuronal activity and that the interpretation of the metabolic signal with regard to cortical neuronal networks must be conducted in a very restrictive manner and concurrent evidence from other experimental approaches be sought. To this date, however, functional MRI is the method that allows the investigation of distributed areas in the human cere-
Figure 4. Results of experiment 3. The sequence of stimulation conditions consisted of: Fixation, Static, Fixation, Motion I (radially moving dots), Fixation, Motion imagery I, Fixation, Motion II (subjective-contour apparent motion stimulus of experiment II), Fixation, Motion imagery II, Fixation, Motion III (rotating grating), Fixation, Motion imagery III, Fixation. (A) Signal time course in cortical area MT/MST averaged over five subjects. The sequence of stimulation conditions (triplets of motion-fixation-imagery) was randomized across subjects but is shown in a fixed sequence. (B) Statistical comparison of activation levels in areas V1, V2, V3A, MTMST, area in inferior parietal lobule (IPL), area in superior parietal lobule (SPL), frontal eye field (FEF) and BA 9/46 averaged over five subjects. The three motion conditions and the three motion imagery conditions are pooled together. Mean percent signal change \pm s.e.m. is displayed for each condition. Activation levels associated with motion imagery increases with the hierarchical level of an area within the dorsal pathway while activation associated with objective motion decreases.

bral cortex that show task dependent coactivation at the highest spatial resolution.

\section{Activation by apparent motion stimuli}

Our results suggest that area MT/MST is the first cortical area along the dorsal stream that is capable of extracting apparent motion cues: In contrast to V1, V2 and V3A, its responses differed significantly between the apparent motion conditions and the respective flickering controls. Comparison of responses to illusory-contour and no-contour conditions suggests that area MT/MST is also capable of using illusory contour information for computing and representing apparent 


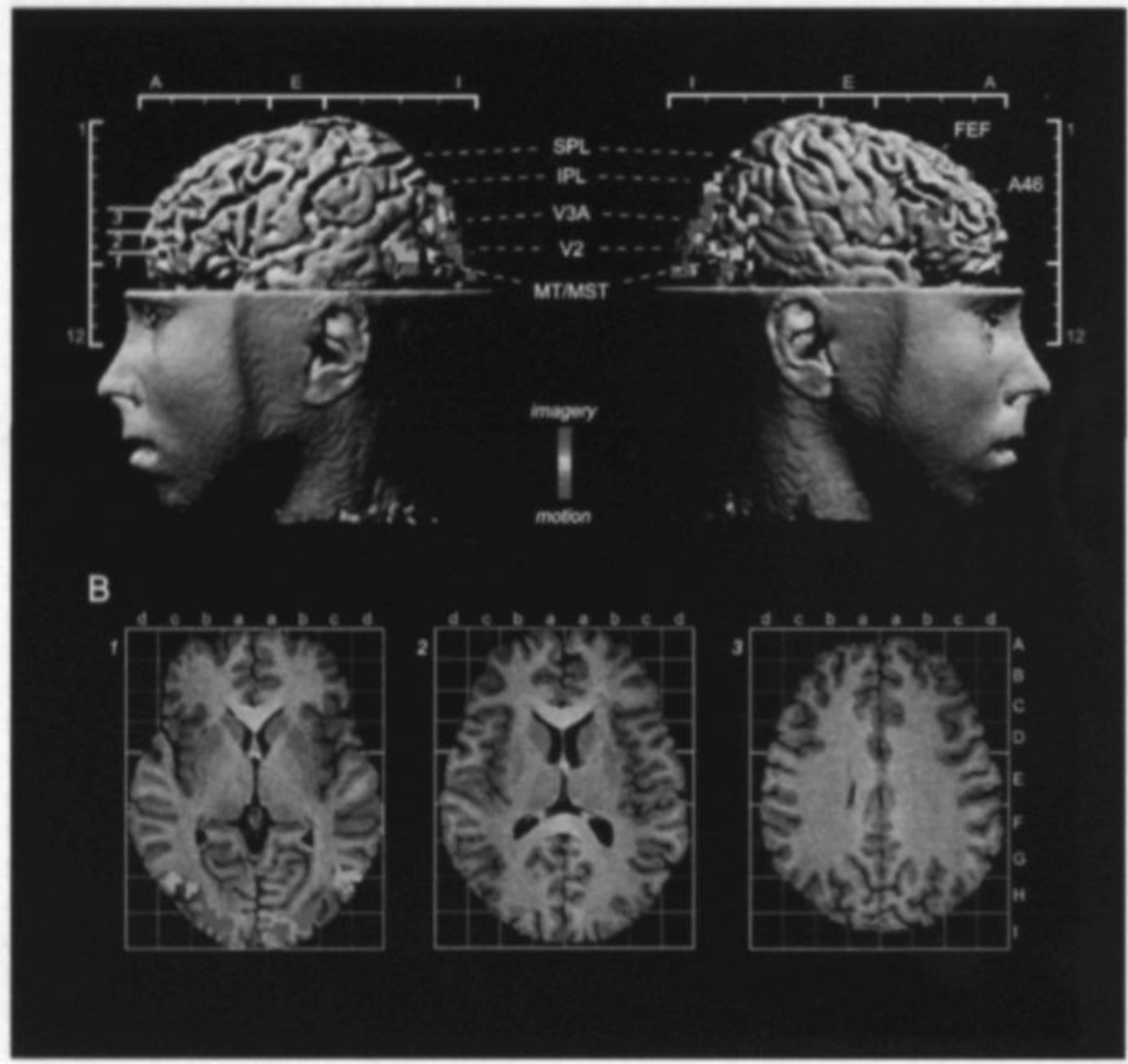

Figure 5. Topological distribution of responses from experiment 3 of one subject. (A) Areas with significant responses during motion and/or motion imagery conditions in a volume rendered view of left (left side) and right (right side) hemisphere. Areas are coloured with respect to a continuum reaching from responses to motion but not to motion imagery (red) to responses to motion imagery but not to motion (green). Intermediate colours depict areas which respond to both conditions (e.g. yellow). White lines with tick marks indicate the coordinate space of the Talairach and Tournoux stereotactic brain atlas. Short yellow lines on left side indicate vertical position of axial slices shown in (B). IPL = area in the inferior parietal lobule, SPL = area in the superior parietal lobule. (B) Selected axial slices as marked in (A) showing several areas with significant responses during motion and motion imagery. Right hemisphere is shown on the left and the left hemisphere is on the right. White lines indicate the coordinate space of the Talairach and Toumaux stereotactic brain atlas. Colouring as in (A). Axial slice 1 shows bilateral activation of area MTMST (yellow), V2 (red) and V3/V3A (red/orange). Axial slice 2 shows activation foci in the insular gyri (green) which can not be seen on lateral view. Axial slice 3 shows BA 9:46 (green) and V3A (orange).

motion. Area $V 2$ responded more strongly to the two illusory-contour conditions than to the two no-contour conditions suggesting that the
Illusory contour information is extracted at that level (von der Heydt et al., 1984, Hirsch 
A
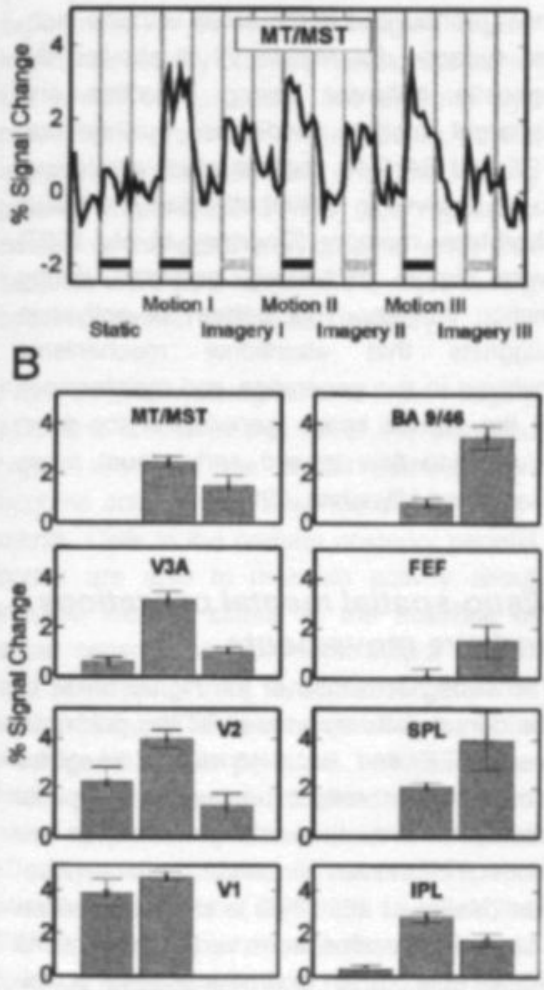

Stafic Motion Imagery
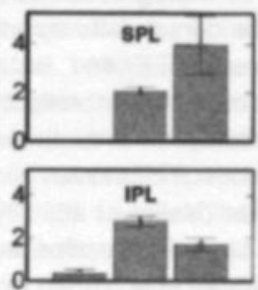

Static Moton Imegery

et al., 1995, ffytche et al., 1996) and made available as input to area MT/MST.

In the second apparent motion experiment. the difference between the apparent motion and the flickering control condition was smaller than in the first two-ring experiment. although the amount of stationary flicker was reduced. One possible explanation is that the opening and closing of the pacmen evoked a motion experience within the same hemifield. Six subjects did in fact report that they had the impression that in the subjective-contour flickering control condition the two Kanizsa squares were shrinking and expanding at their respective location.

Figure 6. Results of experiment 4. The sequence of stimulation conditions consisted of: Fixation, Static I (stationary dots), Fixation, Static imagery (SI) I, Fixation, Motion I (radially moving dots), Fixation, Motion imagery (MI) I. Fixation, Static II (stationary frame of subjective-contour apparent motion stimulus of experiment 2), Fixation, Static imagery II, Fixation, Motion II (Subjective-contour apparent motion of experiment 2), Fixation, Motion imagery III, Fixation. (A) Signal time course in cortical area MT/MST averaged over five subjects. (B) Statistical comparison of activation levels in areas V1, V2, V3A, MTMST, area in inferior parietal lobule (IPL), area in superior parietal lobule (SPL), frontal eye field (FEF) and $B A 9 / 46$ averaged over five subjects. The two static, static imagery. motion and motion imagery conditions are pooled together. Mean percent signal change \pm s.e.m. is displayed for each condition. Activation leveis associated with motion imagery is stronger than activation levels associated with static imagery in most areas of the dorsal pathway. Area MTMST responds during motion and motion imagery conditions but not during static and static imag. ery conditions.

\section{Imagery and perception}

Our finding that $\mathrm{V} 1$ is not significantly active during imagery conditions is in agreement with several PET mental imagery studies (Roland and Gulyás, 1994, Mellet et al., 1996) but is in conflict with a recent PET study (Kosslyn et al., 1995) where significant activation of V1 was found when compared to a non-imagery listening control condition. In Kosslyn's study, however, significant V1 activation was not detectable when compared to the resting baseline condition. Our results suggest that imagery related activation is much less pronounced in V1 than in the 
extrastriate areas V2 and V3A which were identified as significantly activated in our imagery experiments when matched against the visual fixation control condition.

The motion-sensitive MT/MST complex responded strongly when subjects merely imagined moving stimuli. Since the MT/MST complex was selectively activated during motion imagery but not during static imagery. it is unlikely that the activated network of areas during imagery periods is merely the consequence of general activation related to vigilance.

Subjects reported that they were able to evoke mentally a clear motion experience during the imagery periods. At the same time they judged their mental images to contain less figural detail when compared to seeing moving contours. One might speculate that these subjective reports reflect the lack of detectable V1 activity despite the strong activation of MT/MST during motion imagery and that the activation of area MT/MST during motion imagery mediates an experience of motion that is detached from figural details.

In addition to MT and MST, areas at higher levels of the dorsal pathway (Felleman and Van Essen, 1991) were strongly activated during motion imagery and during objective and apparent motion conditions. Although we have no information about the location of monkey areas VIP, LIP and $7 \mathrm{a}$ in humans, the activated regions in the inferior and superior parietal lobule could be the homologues of these areas. Like the activated region in the inferior parietal lobule (IPL, Fig. 4, Fig. 6). monkey area VIP responds to moving stimuli in a similar way as area MT (Colby et al. 1993). The activation of more dorsal parietal regions might be due to covert spatial attention shifts and spatial representations (see below), which would give them a similar function as monkey LIP (Goldberg et al., 1990) and 7a (Andersen et al., 1985). Whilst dorsal pathway activity during motion imagery was most pronounced in the areas with the highest synaptic distance to $V 1$, it showed the opposite behavior during objective and apparent motion conditions. Furthermore, FEF and BA 9/46, regions which are known to be involved in spatial attention and visual short-term memory (Courtney et al., 1997) were almost exclusively activated during motion imagery. This pattern of activation suggests that attentional mechanisms involved in the generation and maintenance of the mental image generate a top-down information flow toward early visual areas (Sakai and Miyashita, 1994).

\section{Visuo-spatial mental operations and eye movements}

The strong activation of the higher areas of the dorsal pathway as well as the prefrontal areas FEF and BA 9/46 during imagined motion, might reflect processes of spatial attention and spatial working memory. In two recent PET studies of mental spatial exploration (Mellet et al., 1995) and spatial mental imagery generated from verbal instructions (Mellet et al., 1996), a similiar bilateral occipitoparietal-frontal network was observed. It is unlikely that the strong activation of any of these areas is a direct consequence of eye movements. In PET studies of voluntary saccadic eye movements (Petit et al., 1993. 1996) activation at the cortical level was found in the precentral gyrus, the supplementary motor area, posterior parietal cortex, the right insula and left cingulate; a weak activation was also found in the right fusiform and lingual gyri. Since this activation pattern matches the results found during apparent motion and motion imagery only partially. we consider a strong contribution of eye movement related activity as unlikely

In order to reduce the occurence of eye 
movements, concentric rings were used as the apparent motion stimulus of the first experiment. In the motion imagery experiments the radially outward moving dot stimulus which did probably not provoke eye movements produced similar results as the apparent motion stimulus and the rotating grating. Experiment 3 was also replicated outside of the scanner in order to verify that subjects were able to fixate the cross during apparent motion and motion imagery.

From electrophysiological and brain imaging studies it is known that all of the activated higher areas of the dorsal pathway can become active in the absence of eye movements. Cells in the primate posterior parietal cortex are able to maintain activity about inferred moving stimuli in the absence of either sensory input or motor output (Assad and Maunsell, 1995). Areas in the superior parietal lobule (SPL) are activated in mental rotation tasks (Cohen et al., 1996) and in tasks which require imagined information about spatial relations in extrapersonal space (Decety et al., 1992) as well as covert spatial attention (Corbetta et al., 1995). Likewise, the FEF is activated when attention is directed towards remembered loci in extrapersonal space (Roland and Skinhoj, 1981) and it has been suggested that the FEF _keeps track of visuospatial information over time" (Raichle. 1989). In the macaque monkey, the FEF is interconnected with the posterior part of the parietal lobe, especially the cortex in the intraparietal sulcus (IPS) which suggests functional coupling of these areas. This is supported by the presented results that the FEF and areas in the inferior and superior parietal lobe are coactivated during motion imagery. Small regions in the dorsolateral prefrontal cortex including BA 9/46 were strongly activated during motion imagery and to a lesser degree during objective motion. and this activation was consistently lateralized to the right hemisphere (Fig. 5). Such a lateralization of BA 9/46 activation to the right hemisphere was also found in association with a spatial working memory task (McCarthy et al., 1994). The activation of the anterior cingulate gyrus might be a correlate of a nonspatial, task-oriented attentional process (Posner and Petersen, 1990) since it was very marked during all mental imagery conditions but almost absent during all other stimulus conditions.

The described network of activated areas indicates that subjects engaged in processes of spatial attention and spatial working memory during motion imagery. The finding that areas of the dorsal processing stream as early as MT/MST are activated by the apparent motion of illusory contours and even by motion imagery nearly as strongly as by real. normally moving contours is an impressive demonstration of the constructive nature of visual processing. Moreover, it implies that these synthetic operations involve the same specialized areas that are held responsible for the analysis of real motion. 


\section{References}

Andersen, R. A. Essick, G. K. and Siegel, R. M. (1985) Encoding of spatial location by posterior parietal neurons. Science, 230, 456-458.

Assad, J. A. and Maunsell, H. R. (1995) Neuronal correlates of inferred motion in primate posterior cortex. Nature, 373, 518-521.

Bandettini, P. A., Jesmanowicz, A., Wong, E. C. and Hyde, J. S. (1993) Processing strategies for time-course data sets in functional MRI of the human brain. Magn Reson Med, 30, 161-173.

Cohen, M. S. Kosslyn, S. M., Breiter, H. C. DiGirolamo, G J., Thompson, W. L., Anderson, A K., Bookheimer, S. Y. Rosen, B. R. and Belliveau, J. W. (1996) Changes in cortical activity during mental rotation - a mapping study using functional MRI. Brain 119, 89-100.

Colby, C. L. Duhamel, J. R. and Goldberg, M. E. (1993) Ventral intraparietal area of the macaque: anatomic location and visual response properties. J Neurophysiol, 69(3), 902-914.

Corbetta, M., Shulman, G. L. Miezin, F. M. Petersen, S. E. (1995) Superior parietal cortex activation during spatial attention shifts and visual feature conjunction. Science, 270, 1423

Courtney, S. M., Ungerleider, L. G. Keil, K. and Haxby J. V. (1997) Transient and sustained activity in a distributed neural system for human working memory. Nature, 386, 608-611.

Cox, R. W. (1996) AFNI: Software for Analysis and Visualization of Functional Magnetic Resonance Neuroimages. Computers and Biomedical Research, 29, 162-173

Decety, J., Kawashima, R. Gulyas, B., Roland, P. E. (1992) Preparation for reaching: a PET study of the participating structures in the human brain. Neuroreport, 3, 761-764

DeYoe, E. A, Carman, G. J., Bandettini, P., Glickman, S. Weser, J., Cox, R., Miller, D. and Neitz, J. (1993) Mapping striate and extrastriate visual areas in human cerebral cortex. Proc Natl Acad Sci USA, 93, 2382-2386.

Engel, S. A, Rumelhart, D. E., Wandell, B. A. Lee, A. T, Glover, G. H. Chichilnisky, E. J. and
Shadlen, M. N. (1994) AMRI of human visual cortex. Nature, 369, 525

Felleman, D. J. and Van Essen, D. C. (1991) Distributed hierarchical processing in the primate cerebral cortex. Cereb Cortex, 1, 1-47.

ffytche, D. H. and Zeki, S. (1996) Brain activation related to the perception of illusory contours. Neurolmage, 3, 104-108.

Goebel, R. (1996) BrainVoyagen a program for analyzing and visualizing functional and structural magnetic resonance data sets. Neurolmage, 3 . S604.

Goebel, R., Muckli, L., Khorram-Sefat, D., Hacker, $H$. and Singer, W. (1996) Going beyond the information given: the neuronal substrate of phimotion and shape-from-motion as revealed by functional magnetic resonance imaging. Neurolmage, 3,5274 .

Goebel, R. (1997) BrainVoyager 2.0: From 2D to 3D fMRI analysis and visualization. Neurolmage. 5, S635.

Goebel, R., Muckli, L., Khorram-Sefat, D., Hacker, H. and Singer, W. (1997) Brain activation during mental imagery of moving stimuli increases with the synaptic distance of an area from V1 along the dorsal processing stream. Neurolmage, 5, S130.

Goldberg, M. E. Colby, C.L. and Duhamel, J. R (1990) Representation of visuomotor space in the parietal lobe of the monkey. Cold Spring Harbor Symposia on Quantitative Biology, LV, 729-739.

Hirsch, J., DeLaPaz, R. L., Relkin, N. R., Victor, J., Kim, K., Li, T., Borden, P., Rubin, N. and Shapley, R. (1995) Illusory contours activate specific regions in human visual cortex. Evidence from functional magnetic resonance imaging. Proc Natl Acad Sci USA, 92, 6469-6473.

Kanizsa, G (1979) Organization in Vision. Essays on Gestalt Perception. New York, Praeger.

Kolers, P. A. (1972) Aspects of Motion Perception. New York, Pergamon Press.

Kosslyn, S. M. Thompson, W. L. Kim, L. J., Alpert. N. M. (1995) Topographical representations of mental images in primary visual cortex. Nature. 378, 496-498. 
McCarthy, G., Blamire, A. M., Puce, A., Nobre, A. C., Bloch, G. Hyder, F., Goldman-Rakic, P. and Shulman, R. G (1994) Functional magnetic resonance imaging of human prefrontal cortex activation during a spatial working memory task. Proc Natl Acad Sci USA, 91, 8690-8694.

McCarthy, G, Spicer, M., Adrignolo, A., Luby, M., Gore, J. and Allison, T (1995) Brain activation associated with visual motion studied by functional magnetic resonance imaging in humans. Human Brain Mapping, 2, 234-243.

Mellet, E., Tzourio, N., Denis, M. and Mazoyer, B (1995) A positron emission tomography study of visual and mental spatial exploration. J Cogn Neurosci, 7, 433-455.

Mellet, E., Tzourio, N., Crivello, F., Joliot, M., Denis, M. and Mazoyer, B (1996) Functional anatomy of spatial mental imagery generated from verbal instructions. J Neurosci, 16, 65046512.

Paus, T. (1996) Location and function of the human frontal eye-field: a selective review. Neuropsychologia, 34, 475-483.

Petit, L., Orssaud, C., Tzourio, N., Salamon, G. Mazoyer, B. and Berthoz, A (1993) PET study of voluntary saccadic eye movements in humans: basal ganglia-thalamocortical system and cingulate cortex involvement. J Neurophysiol, 69, 1009 1017.

Petit, L., Orssaud, C., Tzourio, N., Crivello, F., Berthoz, A., Mazoyer, B. (1996) Functional anatomy of a prelearned sequence of horizontal saccades in humans. J. Neurosci., 16, 3714-26.

Posner, M. I., Petersen, S. E. (1990) The attention system of the human brain. Annu Rev Neurosci, $13,25-42$.

Raichle, M. E. (1989) Developing a functional anatomy of the human brain with positron emission tomography. Curr Neurol, 9, 161-178.

Roland, P. E. and Gulyás, B. (1994) Visual imagery and visual representation. Trends Neurosci, $17,281-287$.

Roland, P. E. and Skinhoj, E. (1981) Extrastriate cortical areas activated during visual discrimination in man. Brain Res, 222, 166-171.
Sakai, K. and Miyashita, Y. (1994) Visual imagery: an interaction between memory retrieval and focal attention. Trends Neurosci, 17, 287-289.

Sereno, M. I., Dale, A. M., Reppas, J. B., Kwong. K. K., Belliveau, J. W. Brady, T. J., Rosen, B. R and Tootell, R. B. H. (1995) Borders of multiple visual areas in humans revealed by functional magnetic resonance imaging. Science, 268, 889 893.

Talairach, J. and Tournaux, P. (1988) Co-planar stereotaxic atlas of the human brain. New York, Thieme.

Tootell, R. B. H., Reppas, J. B., Dale, A. M., Look, R. B., Sereno, M. I., Malach, R., Brady, T. J. and Rosen, B. R. (1995a) Visual motion aftereffect in human cortical area MT revealed by functional magnetic resonance imaging. Nature, 375, 139. 141.

Tootell, R. B. H., Reppas, J. B., Kwong, K. K. Malach, R., Born, R. T., Brady, T. J., Rosen, B. R. and Belliveau, J. W. J. (1995b) Functional analysis of human MT and related visual cortical areas using magnetic resonance imaging. J Neurosci, $15,3215-3230$.

Tootell, R. B. H., Dale, A. M., Sereno, M. I. and Malach, $R$ (1996). New images from human visual cortex. Trends Neurosci, 19, 481-489.

von der Heydt, R., Peterhans, E. and Baumgartner, $G$ (1984) Illusory contours and cortical neuron responses. Science, 224, 1260-1262.

Watson, J. D. G. Myers, R., Frackowiak, R. S. J., Hajnal, J. V., Woods, R. P., Mazziota, J. C., Shipp. S. and Zeki, S (1993) Area V5 of the human brain evidence from a combined study using positron emission tomography and magnetic resonance imaging. Cereb Cortex, 3, 79-94.

Van Essen, D. C. and Drury, H. A. (1997) Structural and functional analyses of human cerebral cortex using a surface-based atlas. I Neurosci, $17,7079-7102$.

Zeki, S., Watson, J. D. G and Frackowiak, R. S. J. (1993) Going beyond the information given: the relation of illusory visual motion to brain activity. Proc R Soc B, 252, 215-222. 
94

\section{Acknowledgements}

This work was supported by a grant from the Academy of Sciences of Berlin-Brandenburg. We thank D.E.J. Linden, M. H.J. Munk and P.R. Roelfsema for valuable comments on the manuscript. T. Dierks for EOG recordings and C.I. Goebel for her help in performing the studies 


\section{Chapter 6}

\section{Apparent motion: Event-related fMRI of perceptual switches and states}

This chapter corresponds to:

Lars Muckii ${ }^{1}$. Nikolaus Kriegeskorte ${ }^{2}$, Heinrich Lanfermann ${ }^{3}$. Friedhelm E. Zanella ${ }^{3}$. Wolf Singer ${ }^{1}$ and Rainer Goebel ${ }^{1.2}$ (2002) Apparent motion: Event-related fMRI of perceptual switches and states. Journal of Neuroscience, 22, RC219 (1-5).

'Max Planck Institute for Brain Research, Neurophysiology. Frankfurt am Main, Germany. 2Department of Psychology, Neurocognition, Universiteit Maastricht, Maastricht, The Netherlands. ${ }^{3}$ Department of Neuroradiology, Johann Wolfgang Goethe-Universitat, Frankfurt am Main, Germany 


\section{Summary}

When spatially segregated visual stimuli are presented in alternation, subjects may perceive a single stimulus moving between the two positions (apparent motion). By adjusting spatial and temporal parameters, an ambiguous condition can be created in which perception of back-and-forth motion alternates with the perception of two stationary blinking stimuli. We presented subjects with such ambiguous stimuli, asked them to signal periods of perceived motion and blinking and measured brain activity with functional magnetic resonance imaging (fMRI). Multiple regression analysis revealed that early visual areas responded with equal strength during both perceptual conditions whereas $\mathrm{hMT}+(\mathrm{V} 5)$ was more active during the perception of apparent motion. These results indicate that neurons in $\mathrm{hMT}+$ participate in the constructive process that creates a continuous motion percept from a discontinuous visual input. 


\section{Introduction}

Moving visual stimuli are known to enhance activity in various regions of the primate brain, particularly in monkey temporal areas MT and MST (Zeki, 1974; Van Essen et al., 1981; Mikami et al., 1986; Tanaka et al. 1986; Dodd et al., 2001) and in hMT+, the human motion complex which includes the human homologue of MT and its satellites (Zeki et al., 1991; Tootell et al., 1995; Sunaert et al., 1999). Such activation has also been demonstrated for stationary flashed stimuli if their timing and spatial arrangement are adjusted to give rise to apparent motion perception (Korte, 1915; Kolers, 1972; Newsome et al., 1986; Mikami, 1991; Goebel et al., 1998). Further evidence for a close relation between perception of visual motion and these areas comes from the demonstration that direct electrical stimulation (Salzman et al., 1992; Salzman and Newsome, 1994), or indirect transcranial magnetic stimulation (Pascual-Leone and Walsh, 2001) of neurons in MT/MST influences motion perception. A common feature of all these stimulation experiments is that changes in perception are studied as the dependent variable following manipulations that alter neuronal responses. Here we treat neuronal activity in the hMT+ as the dependent variable and examine whether it changes as a function of perception even if there is no change of stimulation conditions.

To induce a bistable percept we used two squares presented alternately on either side of the fixation point (Fig. 1). Depending on stimulation parameters subjects perceived either two independently blinking squares or a single square moving back and forth between the two locations. For a particular parameter setting specific for each subject. this stimulus becomes ambiguous: subjects perceive it as moving and blinking in alternation. This phenomenon is also known as apparent motion breakdown effect (De Silva, 1928; Kolers, 1964; Tyler, 1973; Selmes et al., 1997) and has been attributed to neuronal adaptation (Clatworthy and Frisby, 1973. Anstis et al., 1985). In the present fMRI study. stimulus parameters were adjusted for each subject until switches between apparent motion and blinking squares (breakdown and recovery of apparent motion) were reported. Stimulus parameters were then fixed for the recording sessions during which subjects reported perceptual switches by pressing one of two response buttons

\section{Methods}

\section{Stimulus}

The stimulus consisted of two white blinking squares (size: $1.3^{\circ}$ visual angle; contrast $94 \%$; luminance: $6 \mathrm{~cd} / \mathrm{m}^{2}$ ) presented on a dark screen to either side of the fixation point (Fig. 1a). The stimulus parameters varied across subjects as follows: distance between the centers of the squares between $11.7^{\circ}$ and $13.5^{\circ}$ (average: $13^{\circ}$ ), stimulus duration between $116 \mathrm{~ms}$ and $166 \mathrm{~ms}$ (average: 146 $\mathrm{ms}$ ) and interstimulus interval between $50 \mathrm{~ms}$ and $67 \mathrm{~ms}$ (average: $52 \mathrm{~ms}$ ).

The human motion complex was mapped in separate experiments for four subjects comparing responses to 400 stationary white dots with responses to a motion stimulus that consisted of $\mathbf{4 0 0}$ white dots moving radially outward on a dark screen (visual field: $30^{\circ}$ wide by $23^{\circ}$ high, dot size: $0.06^{\circ} \times 0.06^{\circ}$ dot velocity $3.6^{\circ}-14.4^{\circ} / \mathrm{s}$ ). This stimulus is known to produce a clear hMT response (Tootell et al., 1995; Goebel et al., 1998). Stimuli were generated by computer and backprojected onto a frosted screen with an LCD projector. Subjects viewed the screen through a mirror fixed to the head coil. 


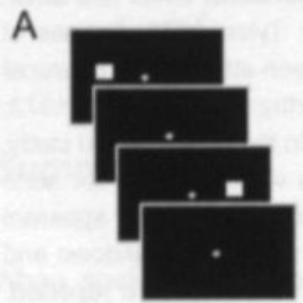

D

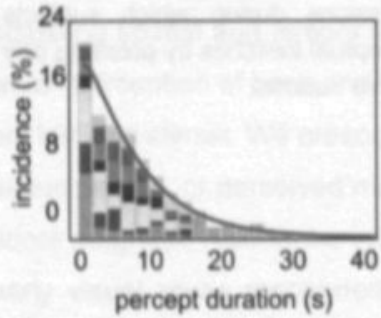

F

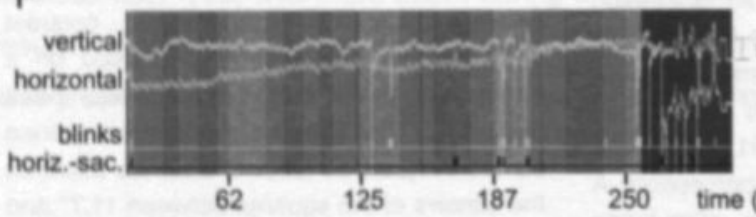

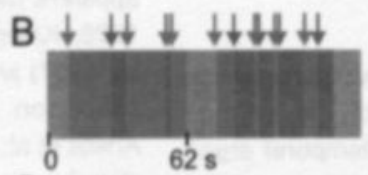

C

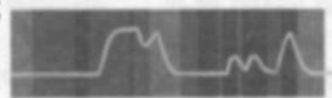

E

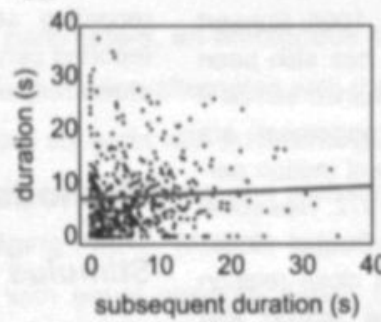

Figure 1.A) The apparent motion stimulus: a white square presented alternately at two positions Stimulus parameters were adjusted for each subject in the scanner until perceptual switches between apparent motion and blinking squares were reported. (B) This bistable stimulus was continuously presented in blocks of $46 \mathrm{~s}$ preceded and followed by 17 s of fixation (gray blocks). Subjects indicated whether they perceived motion (red-periods) or blinking (blue-periods) by pressing one of two response buttons (red and blue arrows). (C) The expected hemodynamic response time course for the separate perceptual conditions (yellow: for

(s) apparent motion). The first

perceptual phase of each stimulation block was represented as a separate predictor in multiple regression analysis and was omitted from further analysis. (D, E) Temporal dynamics of perceptual switches. (D) Histogram of percept durations during MRI-experiments accumulated over all subjects. Each column refers to the acquisition time of one brain volume (2 s). Individual (colored bars) and cumulative

\section{Procedure}

Subjects $(n=8)$ reported their current perceptual state by pressing one of two optic fiber response buttons with their right hand (Fig. 1b). Stimulus presentation lasted $46 \mathrm{~s}$ and was preceded and followed by a $17 \mathrm{~s}$ fixation period during which only the fixation cross was present. Each subject was exposed to 12 stimulation blocks presented in three successive runs of scanning. Subjects were instructed to foxate throughout the experiment. Eye position was controlled in 3 subjects by an infrared eye-tracking system (Ober2, Permobil Meditech, Timra, Sweden). Four subjects participated in a separate experiment designed to map $\mathrm{hMT}+$, in which objective motion and static control stimuli were each presented in 6 blocks of $16 \mathrm{~s}$ and alternated with fixation periods of equal duration.

\section{Functional image acquisition and analysis}

Blood oxygenation level dependent fMRI (BOLD, Ogawa et al., 1990) was acquired with a 1.5 Tesla Magnetom Vision Scanner (Siemens, Erlangen, Germany) using a gradient echo $E P I$ sequence ( 1 volume $=16$ axial slices, repetition time $=2081 \mathrm{~ms}$, echo time $=$ 
$69 \mathrm{~ms}$, flip angle $=90^{\circ}$, field of view $=210 \times$ $210 \mathrm{~mm} 2$, voxel size $=3 \times 3 \times 5 \mathrm{~mm} 3$ ). A high-resolution $\mathrm{T} 1$-weighted anatomical scan was acquired for each subject for reference in single subject analysis. The first slice scan time within a volume was used as a reference for alignment by linear interpolation of the following slices of that volume to correct for the temporal slice scan time shifts. Data analysis and visualization were performed with the fMRI software package BrainVoyager 4 (Braininnovation, Maastricht. The Netherlands; Goebel et al., 1998). Prior to analysis. the data were preprocessed as follows: 1) 3d motion correction; 2) spatial smoothing of EPI images with FWHM of $8 \mathrm{~mm}$ for the group analysis and $4 \mathrm{~mm}$ for the single subject analysis; 3 ) trend removal by temporal FFT. based high pass filtering, removing components with a period longer than $84 \mathrm{~s} ; 4$ ) transformation into Talairach (Talairach and Tournaux, 1988) coordinate space. The cortical sheets of the individual subjects and a template brain were reconstructed as polygon meshes based on the high resolution $\mathrm{T} 1$. weighted structural $3 \mathrm{D}$ recordings. The white/ gray matter boundary was segmented, reconstructed, smoothed, morphed and flattened as described in detail elsewhere (Linden et al., 1999; Kriegeskorte and Goebel, 2001). A morphed surface always possesses a link to the folded reference mesh so that functional data can be correctly projected onto partially inflated as well as flattened representations.

The subjectively defined perceptual phases between two successive switches were used for multiple linear regression analysis of the BOLD signal time course. Using an empirically founded model (Boynton et al., 1996) of the temporal dynamics of the fMRI signal, hemodynamic predictors were computed from the subjects' indications of perceptual phases and a general linear model (GLM) was computed for every voxel (Fig. 1c). To exclude unspecific stimulus onset effects, the first perceptual phase of each stimulation block was excluded. A contrast analysis of the predictors comprising the remaining perceptual phases was used to find regions where average activity was higher during phases of apparent motion perception than during phases of blinking perception in group analysis and single subject analysis of 8 subjects (Fig. 2). Event-related average time courses were computed for each subject. Segments representing the same perceptual conditions were averaged over successive runs (Fig. 3).

In a second GLM analysis the predictors were modeled to comprise only transient switch-related activity. The predictor model was built as described for the phase-related GLM, except that the switch-related predictors had a duration of one volume $(2 \mathrm{~s})$.

The hMT+ was located with a 2 predictor GLM analysis of the objective motion experiment, using one predictor for the radial motion condition and one for the static condition. Contrast analysis between these predictors permitted identification of regions with the highest activity difference between motion and static conditions.

Binocular eye positions were sampled with $100 \mathrm{~Hz}$. Radio-frequency induced artifacts were removed by a sequence triggered threshold algorithm. The traces were analyzed by a threshold based algorithm and calibrated with $5^{\circ}$-reference saccades. This procedure allowed detection of saccades $>1$ " and eye-blinks.

\section{Results}

\section{Psychophysics}

All subjects experienced frequent perceptual transitions during the experiment. Perceptual phases lasted between $2.3 \mathrm{~s}$ and $10.6 \mathrm{~s}$ 
(lower - upper percentile) with an average of $7.7 \mathrm{~s}$ (median: $5.6 \mathrm{~s}$ ). The distributions of phase durations are strongly shiffed toward the left and can be approximated in each individual by a gamma distribution. Six out of eight individual fits showed no significant deviation ( $p>0.2$; Kolmogorov-Smirnov-test) but two fits deviated significantly $(p<0.05$; Fig. 1d). These distributions of phase durations agree with the temporal dynamics of perceptual switches such as occur with binocular rivairy (Levelt, 1965; Walker, 1975; Lehky. 1988; Leopold and Logothetis, 1999) or with ambiguous figures like the Necker cube, or shape from shading (Taylor and Aldridge. 1974). The temporal dynamics of multistable perception are usually characterized by stochastic independence of consecutive phases (Walker, 1975). We confirmed such independence in 6 of 8 subjects (Fig. 1e; r=-0.02; $0.03 ;-0.04 ; 0.12 ;-0.17 ;-0.18$ ). However, 2 subjects exhibited significant dependencies ( $r=-0.31, p<0.01 ; r=0.37, p<0.01)$.

\section{Eye movements}

Horizontal saccades above $1^{\circ}$ were rare and occurred with a frequency of $0.3-0.8 / \mathrm{min}$ during fixation, $0.3-1.9 / \mathrm{min}$ during perceived motion, and $0-1.2 / \mathrm{min}$ during perception of blinking (differences between perceptual conditions were not significant $1<1.5 ; p>0.1$ ). No saccades above $5^{\circ}$ were detected during the IMRI experiments. Vertical saccades and eye blinks occurred more often during the fixation period $(7-19 / \mathrm{min}$; significance in all subjects $1>2.8$; $p<0.01)$ than during the visual stimulation (motion: $1.7-4.3 / \mathrm{min}$; blinking: 0.6-1.1/ $\mathrm{min}$ ). The probability of eye movements was not increased around perceptual switches. On average, the positions of the eyes were equally for both perceptual conditions $(t<0.7$. $p>0.5$ ).

\section{Group analysis}

The GLM group analysis of contrast differ- ences between episodes with differing percepts revealed regions with higher activity during perception of motion than during perception of blinking. Fig. 2 (a, b) shows the clusters with the highest contrast. At this threshold, each voxel in the map is significant at $p<0.0001$ (Bonferroni corrected for 49125 comparisons in voxel space). The reverse contrast does not label a single voxel at this threshold (not shown). By their coordinates in Talairach space (centers of gravity of the clusters: $R H: x=48, y=-72, z=-1 ; L H: x=-47$. $y=-70, z=2$ ) clusters were identified as corresponding to both hemispheres' hMT+.

The GLM group analysis of transient signal changes revealed an increase in activity for both perceptual switches in the primary motor and somatosensory cortex (RH: $x=51 . y=-$ $37 ; z=34 ;$ LH: $x=-46 ; y=-29 ; z=37)$, in the insular cortex (RH: $x=45 ; y=6 ; z=19$; LH: $x=-39$; $y=4 ; z=11$ ), in the right middle frontal cortex $(x=43 ; y=31 ; z=22)$; in the supplementary motor area (SMA) and in the medial anterior cingulate $(x=3 ; y=9 ; z=45)$. Most of this activity can be attributed to the preparation and execution of the right hand motor responses.

\section{Single subject analysis}

Single subjects were analyzed in the same way for areas activated more strongly during the percept of motion than during that of blinking. In agreement with the group analysis, hMT+ was the region that showed the strongest and most consistent difference in activation when the percept switched from blinking to motion. In all 8 subjects, the contrast analysis revealed activation of hMT+. In 5 subjects hMT + was activated only in the left and in 3 in both hemispheres (Fig. 3). The identified hMT+ clusters were subjected to an event-related time-course analysis. BOLD signal was averaged using the subjects' button presses as trigger. This resulted in average signals comprising the volume during 

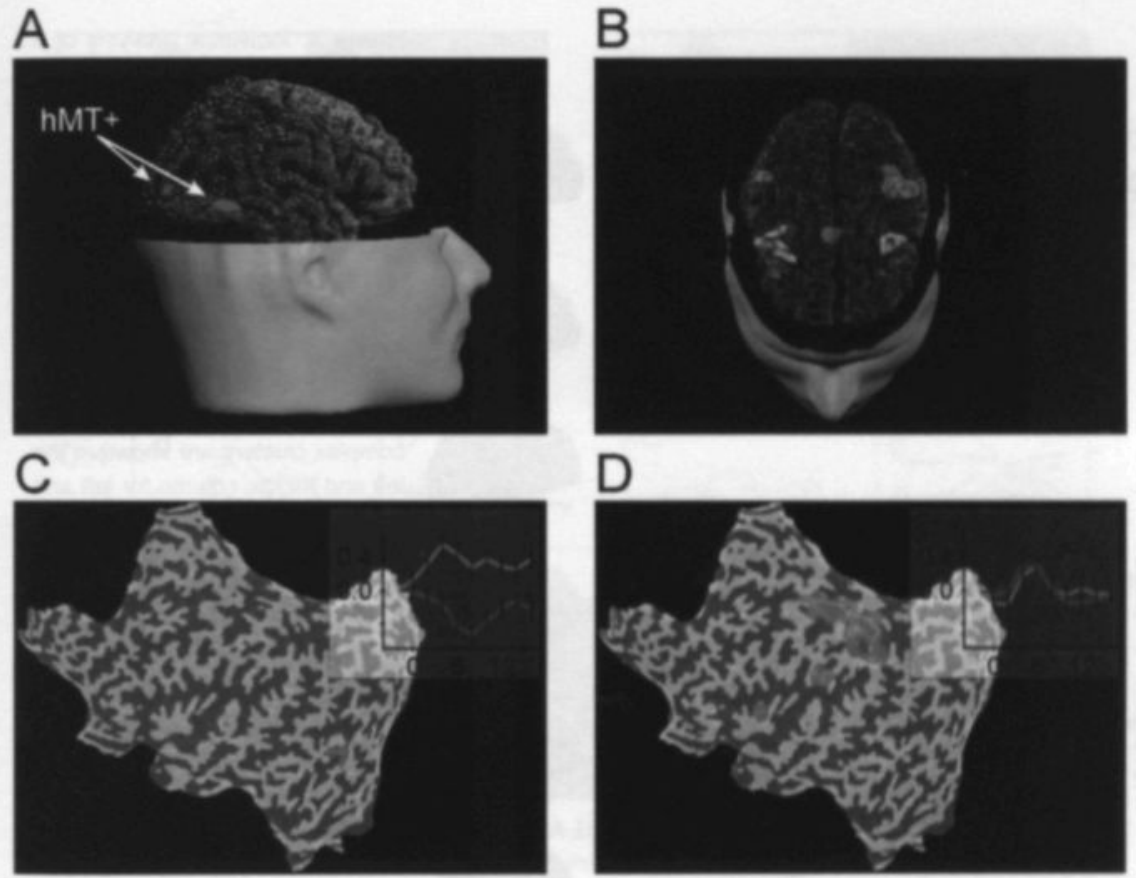

Figure 2. (A, B) Group analysis shown on transparent template brains. (C, D) The corresponding analyses performed for a single subject and presented on flatmaps for the left hemisphere (A, C) GLM-contrast analysis detects regions where activity is higher during the perception of motion than during blink perception. The clusters correspond to the left and right hemisphere's human motion complex. (C) Single subject's flattened hemisphere with the corresponding event-related time course of hMT+. Signal time courses after perceptual switches from blinking to motion are shown in red and after switches from motion to blinking in blue. Dark and light shading indicates gyral structure (dark: concavity; light: convexity). (B, D) Results from the switch-related GLM analysis of group data (B) and single subject data (D). GLM detects regions with transient switch-related activity in motor- and somatosensory cortex, insular cortex, right middle frontal cortex, SMA and middle frontal cortex.

which the perceptual transition occurred plus the eight volumes following the transition (corresponding to an interval of -2 to $+16 \mathrm{~s}$ ). As the next transitions are likely to occur within this time interval and as these switches sometimes occur quite soon subsequent switches tend to blurr the time course of individual responses. Fig. 3 shows these time courses for hMT+ of the left and right hemi- sphere of each subject. The colored bars below the time courses indicate the persistence of the percepts signaled by the subjects. Signal intensity rises after perceptual transitions from blinking to motion (red; $t=$ 3.7 p<0.01) and falls after transitions from motion to blinking (blue; $\mathrm{t}=0.9$; $\mathrm{ns}$ ). The empty panels in Fig. 3 correspond to hemispheres whose hMT+ exhibited no differential 


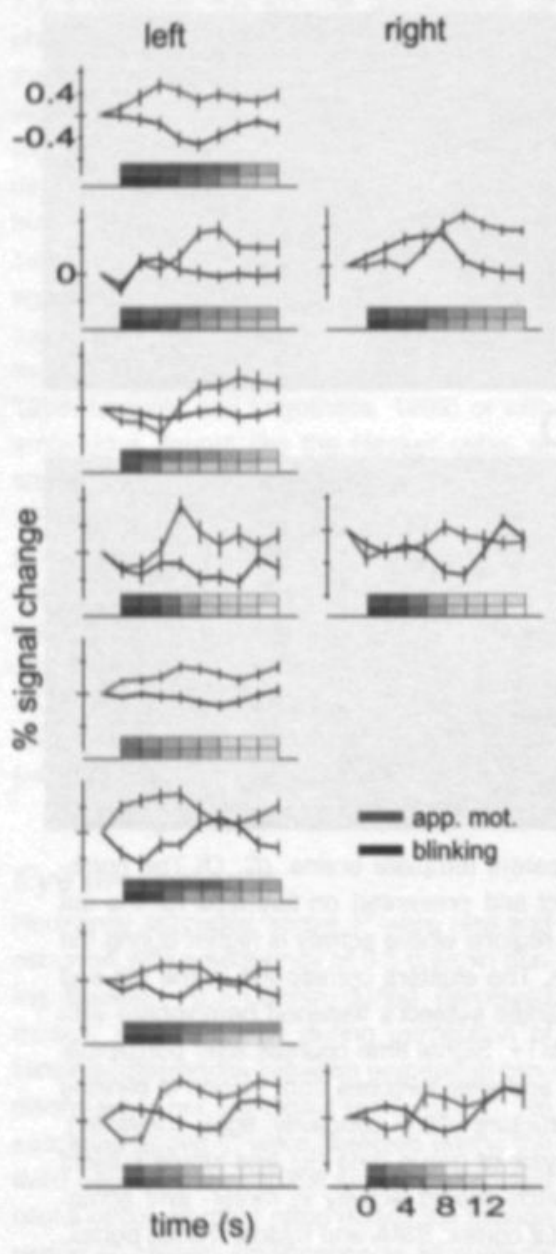

activation during the motion percept. The locations of the selected regions of interest (ROIs) match well with estimates on the location of hMT+, based on cortical morphology (temporo-occipital-boarder of inferior-temporal sulcus, Fig. 3 right column), on Talairach coordinates, or on the results of functional mapping (Fig 4). Despite non-optimal overlap in two out of four subjects (Fig 4 C, D) the time courses showed the same characteristics for functionally mapped hMT+ regions.
Figure 3 . Individual analysis of 8 subjects. In the right column actvated clusters are superimposed onto individual reconstructions of the cortical sheet of the left or right hemisphere. Regions where activity is stronger during motion than during blinking are marked in yellow. Regions with the same activ. ity during both conditions are not marked. Event-related average time courses of human motion complex clusters are shown in the left and middle column for left and right hMT+. (Locations are indicated by red circles). Signal time courses after perceptual switches from blinking to motion are shown in red and after switches from motion to blinking in blue. Whiskers correspond to $\%$ one standard error of the mean. Each of the colored rectangles below the time courses represents a func. tional volume (2s). The color indicates the proportion of averaged time courses, on which the percept endured. Dark color (intensity $100 \%$ ) indicates that the respec. tive percept (motion red; blinking blue) was present at that functional volume on every one of the averaged time courses. White color (intensity 0\%) indicates that the percept had switched again on all averaged time courses.

\section{Discussion}

Combining a bistable visual stimulus, perceived as stationary blinking or moving. with an event-related MRI design revealed neuronal correlates of changes in perception that were not stimulus-driven but must have resulted from changes of internal dispositions. The region exhibiting the strongest 

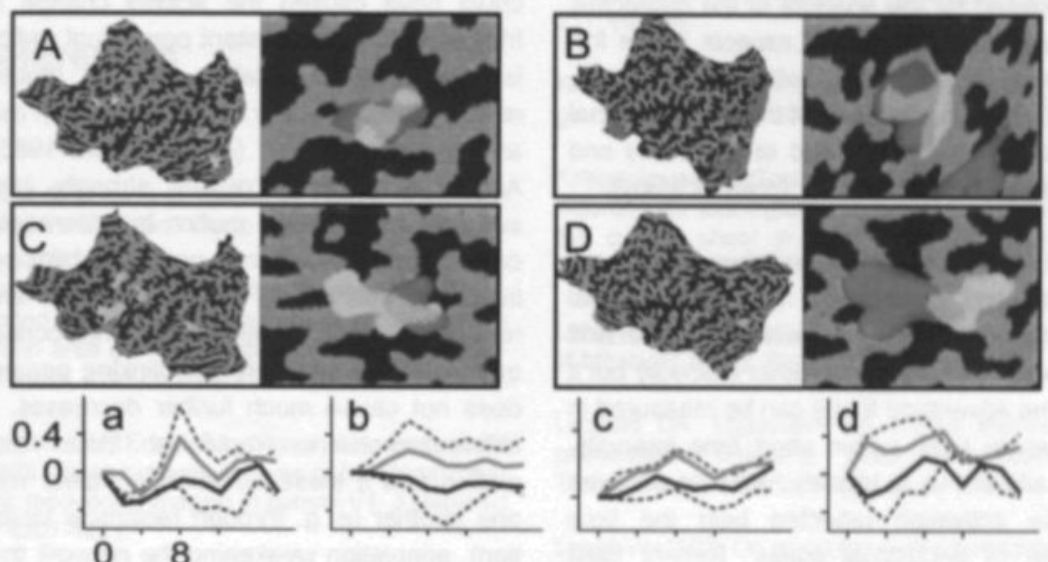

08

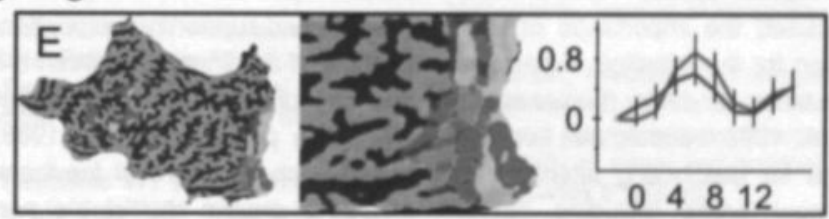

Figure 4. Functional mapping of hMT+ (red). (A-D) Flattened hemispheres of 4 individual subjects with superimposed result of hMT+ mapping. Highest GLM-contrast between objective motion and static dot pattern is shown in orange. Regions with higher activity to apparent motion (ct. Fig. 3) are superimposed in yellow. Both maps overlap largely in hMT+, which is also shown at higher magnification. (a-d) Corresponding event-related time-courses are shown for both patches. Solid lines show time-courses from the functionally mapped hMT+ region (red in A-D), dotted lines are taken from Fig 3 (yellow regions in A-D). (E) Early visual areas in some subjects (shown here for the subject from C) showed transient switch-related activity (green) in regions along the ventral horizontal meridian which is consistent with upper visual field stimulation (Fig. 1a). This activity was not seen in the group analysis (Fig. 2). The event-related time course is from the ventral horizontal meridian within V1 (right panel). Retinotopic mapping of early visual areas as reported in (Goebel, et al. 1998; color codes: V1 and V3 in yellow, V2 and V4 in blue, VP in orange, V3a in dark blue).

change of activity between different phases of perception was the human motion complex. Its activity increased for perceptual switches from stationary to moving patterns. This suggests an important role of hMT+ activity in the conscious perception of motion. In the monkey close relations between neuronal activity and perception have been established exploiting cortical microstimulation (Salzman et al., 1990; Celebrini and Newsome, 1995) or perceptual switches associated with ambiguous figures (Dodd et al., 2001) or binocular rivalry (Logothetis and Schall, 1989). The latter two studies, in particular, permit direct comparison with the present investigation because stimulation conditions were constant and neuronal activity changed in close relation with the objects the monkeys reported to perceive. The present results in human subjects agree well with previously obtained data from monkeys. as both indicate that perceptual switches are associated with strongest changes of neuronal activity in those cortical regions that are 


\section{4}

specialized for the analysis of the respective stimulus qualities: figural aspects in the infero-temporal cortex (Logothetis and Schall, 1989), motion attributes of three dimensional figures in area MT (Dodd et al., 2001) and perceived motion in $\mathrm{hMT}+$ (present study).

What then causes these switches in neuronal activity and perception? The BOLD signal reflects neuronal activity with poor spatial and temporal resolution and rather indirectly but it has the advantage that it can be measured in the whole brain within short time intervals. This allowed us to identify hMT + as the area whose activation reflected best the time course of perceptual states. Recent fMRI studies emphasized the importance of topdown modulation for the initiation of perceptual switches in binocular rivalry (Lumer et al., 1998; Tong et al., 1998; Leopold and Logothetis, 1999) and for distributing attention to specialized visual areas including $\mathrm{hMT}+$ (Buchel and Friston, 1997; O'Craven et al., 1997: Beauchamp et al., 1997; Kleinschmidt et al., 1998). Top-down effects have also been shown in mental imagery. Imagery of a moving pattem is associated with strong activation of both prefrontal cortex and $\mathrm{hMT}+$ and less activation of lower visual areas, while perception of the real stimulus is associated with strong activation of both lower visual areas and $\mathrm{hMT}+$ but less with prefrontal activity (Goebel et al., 1998). However, as there were no comparable correlations between perceptual switches and activation patterns in other areas it is unlikely that the enhanced activity of hMT+ was secondary to enhanced activity in other cortical areas. Therefore, the possibility needs to be considered that the switch in perception from flicker to motion is actually a direct consequence of increased activity in hMT+. Thus, while imagery of motion seems to require top-down activation of hMT+, perception of both real and illusory motion might mainly require bottom-up activation of $\mathrm{hMT}+$. A possible mechanism that could have caused the activity change in hMT + and the concomitant perceptual switch is adaptation. Adaptation to real motion reduces the probability of perceiving both real and apparent motion (Anstis et al., 1985). Activity of $\mathrm{hMT}+$ increased strongly after switches to perceived motion but decreases only little after switches to perceived blinking. indicating that activity of $\mathrm{hMT}+$ adapts and returns to near baseline with the consequence that a switch to the blinking percept does not cause much further decreases. If different networks represent flicker and motion and if these networks compete with one another (e. g. through reciprocal inhibition), adaptation weakening the network that is active and supporting perception - can lead to shifts of dominance between the networks and to concomitant switches between the respective percepts. Lehky (1988) has proposed such a reciprocal feedback inhibition model to explain multistable perception in binocular rivalry. A simulation based on this model reproduced the typical temporal dynamics of perceptual switches, that were also apparent in our behavioral data (Fig. 1de). This and the fact that hMT+ activity correlated best with the perceptual changes suggests that the perceptual switches were due to adaptation and competition within hMT+, and were not gated by top down influences.

Since hMT + was the structure whose activity correlated best with the perceptual changes we propose that the perceptual switches between stationary and moving patterns resulted from changes between mutually exclusive processing strategies that occurred within hMT+. Our data suggest that these changes were not gated by top down influences but occurred as a function of intrinsic processes as e. g. adaptation. 


\section{References}

Anstis S, Giaschi D, Cogan Al (1985) Adaptation to apparent motion. Vision Res 25: 1051-1062.

Beauchamp MS, Cox RW, DeYoe EA (1997) Graded effects of spatial and featural attention on human area MT and associated motion processing areas. J Neurophysiol 78: 516-520.

Boynton GM, Engel SA, Glover GH, Heeger DJ (1996) Linear systems analysis of functional magnetic resonance imaging in human $\mathrm{V} 1$. J Neurosci 16: $4207-4221$

Buchel C, Friston KJ (1997) Modulation of Connectivity in Visual Pathways by Attention: Cortical Interactions Evaluated with Structural Equation Modelling and FMRI. Cereb Cortex 7: 768-778.

Celebrini S. Newsome WT (1995) Microstimulation of extrastriate area MST influences performance on a direction discrimination task. J Neurophysiol 73: 437-448.

Clatworthy JL, Frisby JP (1973) Real and apparent visual movement. Evidence for a unitary mechanism. Perception 2: 161-164.

De Silva HR (1928) Kinematographic movement of parallel lines. J gen Psychol 1: 550-577.

Dodd JV, Krug K, Cumming BG, Parker AJ (2001) Perceptually bistable three-dimensional figures evoke high choice probabilities in cortical area mt. J Neurosci 21: 4809-4821

Goebel R, Khorram-Sefat D. Muckli L, Hacker H. Singer W (1998) The constructive nature of vision: direct evidence from functional magnetic resonance imaging studies of apparent motion and motion imagery. Eur J Neurosci 10: 1563-1573.

Kleinschmidt A, Buchel C, Zeki S, Frackowiak RS (1998) Human brain activity during spontaneously reversing perception of ambiguous figures. Proc R Soc Lond B Biol Sai 265: 2427-2433

Kolers P (1972) Aspects of Motion Perception Oxford and New York: Pergamon Press
Kolers PA (1964) Illusion of movement. Sci Am 211: 98-108.

Korte A (1915) Kinematoskopische Untersuchungen. Z Psychol 194-296

Kriegeskorte N, Goebel R (2001) An efficient algorithm for topologically correct segmentation of the cortical sheet in anatomical MR volumes. Neurolmage 14: 329-346.

Lehky SR (1988) An astable multivibrator model of binocular rivalry. Perception $17: 215-228$.

Leopold DA, Logothetis NK (1999) Multistable phenomena: changing views in perception Trends Cognit Sci 3: 254-264

Levelt W (1965) On binocular rivalry. Soesterberg. Netherlands: Institute of Perception

Linden DE, Prvulovic D, Formisano E, Vollinger M. Zanella FE, Goebel R, Dierks T (1999) The functional neuroanatomy of target detection an AMRI study of visual and auditory oddball tasks. Cereb Cortex 9: 815-823

Logothetis NK, Schall JD (1989) Neuronal correlates of subjective visual perception. Science 245 761-763

Lumer ED, Friston KJ, Rees G (1998) Neural cor. relates of perceptual rivalry in the human brain. Science 280: 1930-1934

Mikami A (1991) Direction selective neurons respond to short-range and long-range apparent motion stimuli in macaque visual area MT. Int $\mathrm{J}$ Neurosci 61: 101-112

Mikami A, Newsome WT, Wurtz RH (1986) Motion selectivity in macaque visual cortex. I Mechanisms of direction and speed selectivity in extrastriate area MT. J Neurophysiol 55: 1308-1327.

Newsome WT, Mikami A. Wurtz RH (1986) Motion selectivity in macaque visual cortex. III. Psychophysics and physiology of apparent motion. J Neurophysiol 55: 1340-1351

O'Craven KM, Rosen BR, Kwong KK, Treisman A, Savoy RL (1997) Voluntary attention modulates AMRI activity in human MT-MST. Neuron 18: 591. 598 
Ogawa S, Lee TM, Kay AR, Tank DW (1990) Brain magnetic resonance imaging with contrast dependent on blood oxygenation. Proc Natl Acad Sci U S A 87: 9868-9872.

Pascual-Leone A, Walsh V (2001) Fast backprojections from the motion to the primary visual area necessary for visual awareness. Science 292: $510-512$.

Salzman CD, Britten KH, Newsome WT (1990) Cortical microstimulation influences perceptual judgements of motion direction. Nature 346: 174 . 177

Salzman CD, Murasugi CM, Britten KH, Newsome WT (1992) Microstimulation in visual area MT. effects on direction discrimination performance. J Neurosci 12: 2331-2355

Salzman CD, Newsome WT (1994) Neural mechanisms for forming a perceptual decision. Science 264: $231-237$

Selmes CM, Fulham WR, Finlay DC. Chortton MC. Manning ML (1997) Time-till-breakdown and scalp electrical potential maps of long-range apparent motion. Percept Psychophys 59: 489. 499.

Sunaert S, Van Hecke P. Marchal G Orban GA (1999) Motion-responsive regions of the human brain. Exp Brain Res 127: 355-370.

Talairach J. Tournaux P (1988) Co-planar Stereotaxic Atlas of the Human Brain. New York. NY Thieme.

Tanaka K, Hikosaka K. Saito H, Yukie M, Fukada $Y$, Iwai E (1986) Analysis of local and wide-field movements in the superior temporal visual areas of the macaque monkey. J Neurosci $6: 134-144$.

Taylor MM, Aldridge KD (1974) Stochastic processes in reversing figure perception. Perception \& Psychophysics 16: 9-27

Tong F, Nakayama K. Vaughan JT, Kanwisher N (1998) Binocular rivaliry and visual awareness in human extrastriate cortex. Neuron 21: $753-759$

Tootell RB, Reppas JB, Kwong KK Malach R, Bom RT. Brady TJ. Rosen BR. Belliveau JW (1995) Functional analysis of human MT and related visual cortical areas using magnetic resonance imaging. $J$ Neurosci $15: 3215-3230$
Tyler CW (1973) Temporal characteristics in apparent movement Omega movement vs. phi movement Q J Exp Psychol 25: 182-192

Van Essen D, Maunsell JH, Bixby JL (1981) The middle temporal visual area in the macaque myeloarchitecture, connections, functional properties and topographic organization. J Comp Neurol 199: 293-326

Walker P (1975) Stochastic properties of binocular rivalry alternations. Perception \& Psychophysics 18: $467-473$

Zeki S. Watson JD, Lueck CJ, Friston KJ, Kennard C. Frackowiak RS (1991) A direct demonstration of functional specialization in human visual cortex. J Neurosci 11: 641-649

Zeki SM (1974) Functional organization of a visual area in the posterior bank of the superior temporal sulcus of the thesus monkey. J Physiol (Lond) 236: $549-573$

\section{Acknowledgements:}

The study was supported by the Max Planck Society and the Consortium for the Investigation of Consciousness. We thank Marcus J. Naumer and especially David E. J. Linden for valuable comments on the manuscript and Stuart Anstis for fruitful discussion initiating this study 
Summary 
Functional magnetic resonance imaging (fMRI) was used in 5 different studies to explore the relationship between brain activity in visual areas and constructive visual processes. We presented visual stimuli that induce illusions (illusory contours, apparent motion, transparent motion) or instructed our subjects to recollect visual scenes (i.e. imagery of moving stimuli) and measured brain activation that correlated to the emergence of the visual content. In addition, we investigated brain activity in relation to impairment of constructive processes in amblyopic and hemianopic patients. Amblyopic subjects suffer from reduced visual acuity in otherwise healthy and properly corrected eyes. Hemianopic patients lack visual awareness in a circumscribed region of their visual field.

A short introduction to functional brain mapping of the visual system is given in Chapter 1, in which some of the other chapters are reviewed and set in the context of ongoing developments concerning multi-species comparisons (human and monkeys) and multi-method brain imaging (fMRI, MEG, EEG).

Vision is a constructive process of the brain that incorporates innate knowledge and acquired experience of the visual world. The consequences of visual experience are studied in amblyopic subjects in Chapter 2. The development of concise vision can be impaired for lifetime if children are continuously exposed to non matching visual input of their eyes, i.e. induced by squinting eyes (strabismus) or by uncorrected difference of refractory power (anisometropic amblyopia). We found that the activity of higher visual areas is degraded if amblyopic adults are stimulated with fine visual stimuli through their amblyopic eye. In contrast, early visual areas are not degraded, indicating that the efficiency with which fine grain visual information is transmitted from early to higher visual areas, is impaired in amblyopia

Lesions in the primary visual cortex lead to scotomas - perceptual blind spots in the visual field. Hemianopic subjects studied in Chapter 3 suffer from blind visual fields that extend over a whole hemi-field. Stimulation within these blind fields induces residual visual functions and content specific activation. If hemianopic subjects are stimulated with moving objects, human motion complex ( $\mathrm{hMT}+$ ) is activated, and if they are stimulated with pictures of objects, the ventral visual cortex (LO/V8) is activated. The subjects however remain unaware of either stimulation

Integration of multiple motion vectors over space is needed for the segmentation of natural visual scenes and for reliable motion perception. In Chapter 4 , motion integration is studied in the context of transparent motion perception. In order to evoke the percept of transparent motion, coherently moving dots need to be bound together perceptually and 
assigned to the same surface- a process that can be compromised by other nearby dots. We used small variation of moving dot displays to discriminate visual areas that respond to the basic feature of the stimulus, and areas that respond to a global percept of transparent motion after the integration of multiple motion vectors.

Since the pioneering work of the gestalt psychologists, several rules of perceptual organization have been formulated which characterize visual perception as a constructive process of the brain. A convincing demonstration of the constructive nature of vision can be given by perceptual illusions, e. g. the perception of illusory contours or the perception of apparent motion. Chapter 5 explores how brain activity reflects constructive processes of vision as observed during visual illusions or during mental imagery. This study shows that illusory contours emerge at the cortical processing stage of V2 whereas apparent motion emerges at $\mathrm{hMT}+\mathrm{N} 5$. Apparent motion and motion imagery are accomplished within the same specialized areas that are held responsible for the analysis for real motion.

In all of the preceding studies functional imaging was used to establish links between changes of brain activation in response to different visual stimulation or different instructions. In Chapter 6 we examined whether brain activation changes as a function of spontaneously occurring perceptual switches, even if there are no changes of the stimulation. We presented subjects an ambiguous stimulus that is either perceived as moving back-and-forth (apparent motion) or as two stationary blinking stimuli. We found that activity at the level of hMT+N5 changes in response to the perceptual switches although the visual stimulation remained constant. This allowed us to propose a close link between neuronal activity in $\mathrm{hMT}+\mathrm{N} 5$ and the constructive process that creates a continuous motion percept from a discontinuous visual input. 

Samenvatting 
In 5 verschillende studies werd functionele magnetische resonantie imaging ( $\mathrm{MMRI}$ ) gebruikt om de relatie tussen hersenaktiviteit in visuele gebieden en constructieve visuele processen te verkennen. We boden visuele stimuli aan die illusies opwekken (denkbeeldige contouren, schijnbare beweging, transparante beweging) of instrueerden onze proefpersonen om zich visuele beelden te herinneren (d.w.z. inbeelding van bewegende stimuli) en maten hersenaktiviteit die correleerde met het verschijnen van de visuele inhoud. Daarnaast onderzochten we hersenaktiviteit in relatie tot verstoring van constructieve processen in amblyope en hemianopische patienten. Amblyope proefpersonen lijden aan verminderde visuele scherpte in voor de rest gezonde en goed gecorrigeerde ogen Hemianopische patienten hebben gebrek aan visueel bewustzijn in een omschreven gebied van hun visuele veld.

Een korte inleiding in functionele hersenimaging van het visuele systeem wordt gegeven in hoofdstuk 1, waarin sommige van de andere hoofdstukken besproken worden en in de context van lopende ontwikkelingen op het gebied van vergelijking tussen soorten (mens en aap) en multi-methoden hersenafbeelding (MRI, MEG EEG) geplaatst worden.

Zien is een constructief proces van het brein dat aangeboren kennis en opgedane ervaring omvat. In hoofdstuk 2 worden de gevolgen van visuele ervaring in amblyope proefpersonen bestudeerd. De ontwikkeling van scherp zien kan levenslang verstoord worden wanneer kinderen voortdurend blootgest- eld worden aan niet overeenkomstige visuele input van hun ogen, d.w.z opgewekt door scheelkijkende ogen (strabismus) of door ongecorrigeerde verschillen van refractaire power (anisometropische amblyopie). We vonden dat de aktiviteit in hogere visuele gebieden gedegradeerd is wanneer amblyope volwassenen gedetailleerde visuele stimuli via hun amblyope oog aangeboden krijgen. Vroege visuele gebieden daarentegen, zijn niet gedegradeerd, hetgeen erop wijst dat de efficientie waarmee fijnmazige visuele informatie van vroege naar hogere visuele gebieden doorgegeven wordt verstoord is bij amblyopie.

Beschadigingen in de primaire visuele cortex leiden tot scotoma's - perceptueel blinde vlekken in het visuele veld. Hemianopische proefpersonen, die in hoofdstuk 3 bestudeerd worden, lijden aan blinde visuele velden die een heel hemiveld omvatten. Stimulatie binnen deze blinde velden wekt residuele visuele functies en inhoudsspecifieke aktivatie op. Als hemianopische patienten bewegende objecten aangeboden krijgen, is het human motion complex (hMT+N5) geactiveerd, en als ze plaatjes van objecten aangeboden krijgen is de ventrale visuele cortex geactiveerd (LO/N8). Van geen van beide stimuli zijn de proefpersonen zich echter bewust.

Integratie van meerdere bewegingsvectoren in de ruimte is nodig voor de segmentatie van natuurlijke visuele taferelen en voor betrouwbare bewegingswaarneming. In hoofdstuk 4 wordt de integratie van beweging bestudeerd aan de hand van de waarneming van transparante beweging. Om de waarneming van 
transparante beweging op te wekken, moeten coherent bewegende stippen perceptueel met elkaar verbonden worden en toegeschreven worden aan hetzelfde vlak - een proces dat bemoeilijkt kan worden door andere nabijgelegen stippen. Hetgeen het voor ons mogelijk maakte om visuele gebieden die reageren op het basale kenmerk van de stimulus enerzijds, en gebieden die reageren op een globaal percept van transparante beweging na de integratie van meerdere bewegingsvectoren anderzijds, te onderscheiden.

Sinds het pionierswerk van de Gestalt psychologie, zijn er verschillende regels van perceptuele organisatie geformuleerd die visuele waarneming karakteriseren als een constructief proces van de hersenen. Een overtuigende demonstratie van de constructieve aard van zien kan gegeven worden door perceptuele illusies, bijvoorbeeld de waarneming van illusionaire contouren of de waarneming van schijnbare beweging. Hoofdstuk 5 verkent hoe hersenaktiviteit constructieve processen van het zien, zoals waar te nemen tijdens visuele illusies of mentale inbeelding, reflecteert. Deze studie geeft aan dat illusionaire contouren ontstaan in het corticale verwerkingsstadium van V2, terwijl schijnbare beweging in hMT+N5 ontstaat. Schijnbare beweging en inbeeIding van beweging worden tot stand gebracht in dezelfde gespecialiseerde gebieden die verantwoordelijk gehouden worden voor de analyse van echte beweging.

In elk van de voorgaande studies werd functionele imaging gebruikt om verbanden tussen veranderingen in hersenaktivatie in reaktie op verschillende visuele stimuli of verschillende instructies te leggen. In hoofdstuk 6 onderzochten we of hersenaktivatie verandert als functie van spontaan plaatsvindende perceptuele 'switches', zelfs als de stimuli ongewijzigd blijven. We boden proefpersonen een ambigue stimulus aan die of als heen- en weer bewegend, of als twee stationair flikkerende stimuli waargenomen wordt. We vonden dat aktiviteit op het niveau van $\mathrm{hMT}+$ N5 in reaktie op de perceptuele switches fluctueerde, hoewel de visuele stimuli constant bleven. Dit maakte het voor ons mogelijk om een nauw verband te veronderstellen tussen neuronale aktiviteit in hMT+N5 en het constructieve proces dat een continue waarneming van beweging creeert op grond van een discontinue visuele input. 



\section{Acknowledgments}

For support and advice during the realization of the thesis I thank my advisors and promotors Professor Dr. Wolf Singer and Professor Dr. Rainer Goebel. It has been a pleasure to work with two such inspiring personalities. I greatly appreciated the academic freedom they encouraged, and I am indebted to them for their valuable criticism and support.

More personally, I wish to thank Rainer Goebel for his friendship and to express here my admiration for his constant enthusiasm, which has been an enormous source of inspiration. I have very much appreciated the delightful and productive atmosphere in Wolf Singer's department: the diversity and understanding fostered here are ideal conditions for innovation and success.

I thank all the coauthors and colleagues who contributed to the individual studies they have been acknowledged in the corresponding chapters of this thesis. Special thanks for exciting cooperation go out to Professor Dr. Petra Stoerig. Prof. Dr. Ruxandra Sireteanu and Professor Dr. Michael Scherg. I am indebted to my colleagues Dr. Dr. David E. J. Linden and Dr. Matthias H. J. Munk for invaluable assistance and advice. My thanks also to Nikolaus Kriegeskorte, Stefan Kieß, Marcus J. Naumer and Ariane Fiesser for their help and to my parents for their support. Renate Ruhl's commitment in helping to prepare posters and slides, even in nightshifts or at weekends deserves special thanks. Milene Bonte prepared the dutch translation of my summary. for which I am very thankful.

I am for ever grateful to Amalia Ch. Theodorakopoulos. 


\section{CURRICULUM VITAE}

1969

Born in Aachen, Germany

1991-97 Study of Psychology, Justus-Liebig University Giessen, Germany. Additional studies in Medicine, Applied Mathematics with Computer Sciences, Philosophy.

1994-95 Study of Cognitive Science at the University College London, UK.

1996

Diplom student at the Max-Planck-Institute for Brain Research Dept. Neurophysiology (Prof. W. Singer), functional imaging group (Dr. R. Goebel)

March 1997 University Diploma in Psychology, Justus-Liebig University Giessen, Germany. Thesis : Blindsight in Normal Observers, Functional Magnetic Resonance Imaging for the Investigation of Neurophysiological Correlates of Visual Perception.

March 1998 German Students Award, Körber Foundation

1997-00 Max-Planck-Institute for Brain Research, Ph.D Student under the supervision of Dr. R. Goebel and Prof Dr. W. Singer

March 2000 Max-Planck-Institute for Brain Research, Post-Doc Position

July 2000 Max-Planck-Institute for Brain Research, Staff Position 



\section{SHAKER VERLAG}

ISBN 3-8322-0290-0 